\title{
FLUTTER OF CYLINDRICAL SHELLS \\ CONVEYING FLUID
}

\author{
by \\ Jean-Paul Denise \\ Department of Mechanical Engineering \\ McGil1 University
}

Under the supervision of Dr. M.P. Paidoussis

Submitted to the Faculty of Graduate Studies and Research of McGill University in partial fulfilment of the requirements for the degree of Master of Engineering 


\section{AVANT-PROPOS}

Quand la vitesse d'êcoulement à l'intêrieur d'une enveloppe cylindrique à base circulaire, dont les extrémités sont encastrées ou libres, atteint une certaine valeur critique, l'enveloppe se met à vibrer spontanément et présente une forme correspondant à un second mode circonférentiel de vibration.

Cette thèse dêcrit ce phênomène, apparemment nouveau, et propose une êtude thêorique. Bien que les vibrations observêes soient typiquement non-linéaires, une théorie linéaire prédit de façon satisfaisante le comportement du système, pour des vitesses d'écoulement inférieures ou égales à celle du seuil d'instabilité. Le modèle mathématique est basé d'une part sur les équations de voiles minces de Flügge, permettant la description des mouvements de l'enveloppe, d'autre part sur une théorie classique d'écoulement à potentiel de vitesse pour tenir compte du couplage avec les forces d'origine hydrodynamique.

Cette théorie prédit l'existence de flambage et de vibrations dues à un couplage de modes, dans le cas d'une enveloppe encastrée à ses deux extremitês; dans le cas d'une enveloppe encastrée-libre, des vibrations spontanées apparaissent. La théorie et l'expérience sont en accord qualitatif et quantitatif. 


\section{$\underline{\text { ABSTRACT }}$}

When the flow velocity in a circular cylindrical shell - either cantilevered or with both ends clamped exceeds a certain critical value, flutter of the shell in its second circumferential mode develops spontaneously. This thesis describes this phenomenon, which is believed to be new, and presents a theory used for its study. Although the observed flutter is basically non-linear, a linear theory provides an adequate description of the behaviour of the system, to flow velocities up to and including the . instability threshold. The mathematical model is based on Flügge's shell equations for the description of shell motion and a classical, potential-flow theory to account for the coupled hydrodynamic forces.

This theory predicts the existence of buckling instability and coupled-mode flutter in the case of clampedclamped shells; for cantilevers, self-excited vibrations are predicted. Theory and experiment are both in qualitative and quantitative agreement. 


\section{ACKNOWLEDGEMENTS}

It is a pleasure to acknowledge and thank my research director, Dr. M.P. Paidoussis, for his valuable suggestions and criticisms and the continuous assistance and encouragement which he has offered during the course of this work.

$$
\text { I express my thanks to Mr. J. Baribeau for }
$$

several fruitful discussions, to Mr. M. Hannoyer for helping me with the computational task, to Mr. E. Jones for his help in completing the experimental programme.

I am grateful to the National Research Council of Canada for financial assistance and also acknowledge the support given me as a holder of a France-Québec scholarship. 
IIST OF SYMBOLS

CHAPTER I

INTRODUCTION

CHAPTER II

PROBLEM FORMULATION ? 6

2.1 The Physical System 6

2.2 The Mathematical Model 7

BASIC EQUATIONS AND BOUNDARY CONDITIONS

3.1 Fundamental Equations of Cylindrical Shelis

3.2 Natural Frequencies of Clamped-Clamped or Clamped-Free Circular Cylindrical Shells.

3.3 Influence of Fluid Flow

(a) Pressurization and Curvature Effects

(b) Equations of Fluid Flow . 21

3.4 General Solution $\quad 24$

CHAPTER IV

THEORETICAI RESULTS

4.1 Free Vibration Characteristics of Clamped-Clamped and Clamped-Free Shells in Vacuo

(a) Clamped-Clamped Cylinders

(b) Clamped-Free Cylinders

4.2 Vibration Characteristics in the Presence of Fluid Flow

(a) Clamped-Clamped Cylinders

(b) Clamped-Free Cylinders

(c) Physical Interpretation of the Theoretical Results 
CHAPTER V

EXPERIMENTS

5.1 Introduction 51

5.2 Apparatus 51

5.3 General Observations 53

5.4 Measurements of the Flutter

Thresholds 56

CHAPTER VI

CONCLUSION 60

REFERENCES

APPENDIX A

Small Rigid-Body Motions

APPENDIX B

Natural Frequencies of Thin Circular

Cylindrical shells in Vacuo

APPENDIX C

Vibrations of the Pipe with Flow

APPENDIX D

Eigenfunctions in the Presence of Flow APPENDIX E

Numerical Method - Program Sample 


\section{IIST OF SYMBOLS}

a

h

k.

$\ell$

m

$\mathrm{n}$

$u, v, w$

$N_{X}, Q_{X}$, etc.

$M_{X}, M_{x} \theta$, etc.

$S_{x}, T_{x}$

$\mathrm{x}$

$\theta$

t

$\lambda$ or $\lambda_{j}$

$\omega$

$\Omega$

$\gamma$

U

$\overline{\mathrm{U}}$

E

$v$

$\rho_{\mathbf{s}}$

$\rho$
Mean radius of cylinder

Thickness of cylinder wall

$\mathrm{h}^{2} / 12 \mathrm{a}^{2}$

Length of cylinder

Number of axial half-waves

Number of circumferential waves

Iongitudinal, circumferential and

radial displacement components

stress resultants

Stress couples

Kirchoff's effective shearing

stress resultants

Axial coordinate

Circumferential coordinate

Time

Axial wave number or characteristic root

Circular frequency

Dimensionless frequency

$a^{2} \rho_{s}\left(1-v^{2}\right) / E$

Fluid flow velocity

Dimensionless fluid flow velocity

Young's modulus of elasticity

Poisson's ratio

Density of shell material

Fluid density 
$p$

$I_{n}$

$\mathrm{K}_{\mathrm{n}}$

$\Psi, \phi$
Fluid pressure

Modified Bessel function of first kind, order $n$

Modified Bessel function of second kind, order $n$

Flow potentials 


\section{CHAPTER I}

\section{INTRODUCTION}

The following study is concerned with the flutter of cylindrical shells containing flowing fluid. This phenomenon was accidentally discovered, in March 1969, by Dr. Paidoussis, who was performing experiments on lateral oscillations of tubular cantilevers conveying fluid.

This problem of lateral oscillations of flexible tubes conveying fluid is as follows: when the velocity of fluid flow in a tube, clamped at the upstream end and free at the other, is increased beyond a certain critical value, the system becomes unstable and small random perturbations grow into lateral oscillations of large amplitude. This problem has received considerable attention in the past. In 1953, the Danish scientist, Niordson(1), investigated the vibrations of such a system, his aim being to estimate the natural frequencies of large steel tubes conveying water from the Aswan Dam to the power plant. He concluded that the natural frequencies of bending vibrations always decrease with flow velocity and that there exists a critical velocity corresponding to a buckling form of instability. (Buckling means large deflection of the tube, without oscillation.) 
In 1961, Dr. T.B. Benjamin ${ }^{(2)}$, studying articulated pipe systems conveying fluid, was the first to report on the phenomenon of unstable oscillations, which is possible when such systems possess one free end. His analysis, dealing only with a small number of degrees of freedom, was extended to continuously flexible pipes by Dr. M.P. Paidoussis ${ }^{(3)}$ who established conditions of stability for a cantilever pipe constrained to move in a horizontal plane.

The phenomenon of flutter was observed from the study of such lateral oscillations. It occurred in the following way:

Consider a flexible rubber tube, clamped at one end and free at the other conveying air. If the velocity of air flow is increased, the tube will become unstable and oscillate laterally. If the velocity is increased further, all of a sudden the tube will vibrate in what appears to be a shell-type vibration. These particular vibrations are no longer lateral oscillations of the tube, but periodic deformations of the cross-section of the tube, superposed on the flexural instability. With shorter cantilevers, which are free of flexural instabilities up to high flow velocities, these shell-type vibrations can develop while the cantilever is still straight and stable 
in flexure.

The characteristic features of this flutter phenomenon are the following:

1. Inception - the vibrations may develop spontaneously if the flow velocity is increased beyond a certain critical value; otherwise, they may be induced by pinching or disturbing the tube, in which case they occur in a range of flow velocities below the critical and persist to considerably lower flow velocities.

2. Shape - when vibrating, the free end crosssection presents a shape corresponding to the second circumferential mode of the shell with no flow. At low flow velocities, this shape is regular (see photographs of a silastic clamped-free tube), whereas at higher flow velocities, it becomes rather wobbly. The amplitude is quite large and in the ensuing limit-cycle, the tube opening at the free end closes almost completely at the extremes of the cycle, the lips deforming alternately inward and outward.

3. Iocation - the vibrations are concentrated near the free end and do not extend beyond a few diameters from it.

4. Frequency - the frequency of vibration ranges between $100 \mathrm{~Hz}$ and $800 \mathrm{~Hz}$, producing a shrill sound which can be recorded with a microphone (see experimental 
sound frequencies results).

5. Velocity - the critical velocities of air flow range between $30 \mathrm{ft} / \mathrm{sec}$. and $100 \mathrm{ft} / \mathrm{sec}$. for the tubes used, and the outlet pressure is the atmospheric pressure. The air flow is a fully-developed turbulent one, the Reynolds number being generally of order $10^{4}$.

This phenomenon has thus been observed with a cantilever discharging air. Subsequent experiments indicated that it also occurs when both ends of the tube are clamped, and here again, as in the clamped-free case, the visible deformations are concentrated near the downstream end of the tube. This phenomenon does not seem to occur with water. Experiments were carried out with water; lateral oscillations were observed, but flutter did not happen.

The problem of unstable oscillations of flexible tubes exposed to either an internal or an external inviscid flow has received considerable attention in the past. The flutter and aeroelastic stability of cylindrical shells in the stream of an inviscid fluid has also been considered by several authors including V.V. Bolotin (4), J.W. Miles(5), E.H. Dowell ${ }^{(6)}$ and E.P. Kudryavtsev ${ }^{(7)}$. Bolotin has studied the case of an infinitely long cylindrical shell, using potential flow theory. Although the equations are derived for various types of compressible gas flow (external and 
internal, subsonic or supersonic), the applications are limited to external, supersonic flow. Miles has examined the supersonic flutter using Timoshenko's ${ }^{(8)}$ shell equations. Dowell has considered both subsonic and supersonic flutter of infinitely long shells and discussed qualitatively the case of a shell of finite length; for this latter case, he has indicated that aerodynamic buckling will be the most important type of instability for external subsonic flow. Finally, Kudryavtsev has studied the flutter of elastic cylindrical co-axial shells of infinite length between which flows a compressible fluid, giving, as an example, some results in the case of an absolutely rigid outer shell.

It seems, therefore, at least to the author's knowledge, that the flutter in the case of internal, subsonic flow in a cylindrical shell of finite length has not been reported and studied so far. It is the aim of this research to describe and explain this apparently new phenomenon. 


\section{CHAPTER II}

\section{PROBLEM FORMULATION}

\section{I The Physical System}

Before an analytical study can be attempteä, a mathematical model encompassing the major physical characteristics of the system must be constructed. The physical system under consideration here consists of a flexible cylindrical tube and a subsonic air flow. The operative forces belong to the following three classes:

i) inertial forces;

ii) elastic forces;

iii) aerodynamic forces. The aerodynamic forces are not simply fixed driving forces which excite the elastic structure, but are directly affected by the elastic displacements. Therefore, the phenomenon under study takes on the specific features of an aeroelastic phenomenon, which justifies the name "flutter" given to it. Two major characteristics of the physical system are:
i) non-linearity,

ii) coupling.

The system is non-linear because the vibrations have a large amplitude. Therefore, the equations governing the 
vibrations of the shell are non-linear, as well as the fluid equations. There exists a coupling between the fluid and the tube. When the tube vibrates it deforms and thus influences the flow of the fluid. Reciprocally, the fluid acts through its pressure on the wall and influences the vibrations of the tube.

\subsection{The Mathematical Model}

Based on the physical characteristics of the system, a mathematical model may now be constructed. Although this model should encompass all of the physical characteristics of the phenomenon, the complexity of the system necessitates some simplification, which of course, should be consistent with the physical situation. The principal difficulty arises from the non-linearity of the system. To study the finite deflections of the tube, only a non-linear theory is applicable. Such theories exist, but they are not reliable as little attention has been devoted to them until recently, and moreover they involve complicated equations which can only be solved in simple cases. Therefore, it is ciear that some drastic simplification has to be made.

The important characteristics of the mathematical model will be taken as follows:

1. We shall consider the limiting case of small vibrations. This means that we shall be able to describe 
the behaviour of the system up to and including the threshold of instability. This simplification has been used to determine the conditions of stability in the case of lateral oscillations of cylindrical tubes conveying fluid, and has proven capable of predicting the onset of instability fairly well. The important consequences of this simplification are the following: -

i) a linear shell theory will be used;

ii) the boundary conditions will be taken on the walls of the tube considered to be in an undeformed state;

iii) the tube walls will be considered to be purely elastic with constant elastic properties (Hooke's law).

2. We shall consider the air flow as a potential and incompressible flow. This means the fluid is:

i) non-viscous,

ii) non-heat-conducting,

iii) incompressible.

We assume the laminar sub-layer to be of negligible importance. These assumptions of course, neglect certain physical effects, in particular possible secondary flows at the outlet in the case of a clamped-free tube, but it is felt that the model retains the essential physical 
characteristics of the system and that neglected effects are secondary in importance. 


\section{CHAPTER III}

\section{BASIC EQUATIONS AND BOUNDARY CONDITIONS}

The basic characteristics of the mathematical model having been described, the present chapter proceeds as follows:

First, the fundamental equations of cylindrical shells are derived. This is followed by the determination of the natural frequencies of cylindrical shells with ends either clamped or free. The effects of fluid flow inside the tube are then introduced, followed by the general solution corresponding to vibrations with flow.

\subsection{Fundamental Equations of Cylindrical Shells}

The fundamental equations of cylindrical shells are derived in three steps, as follows:

i) the equations of motion are obtained from a balance of the forces acting on some fundamental element of the medium considered;

ii) the strain-displacement relations are obtained from a geometrical consideration of the process of deformation;

iii) the stress-strain relations are provided by the law of elasticity (Hooke's law). 
The solution of problems in the threedimensional theory of elasticity involves serious complications; thus simplifying assumptions consistent with the behaviour of shells are needed. The basic assumptions of the classical theory were proposed by Love (9) iri 1888. They are the following:

1. The thickness of the shell is small compared with the least radius of curvature of the reference surface; 2. The strains and displacements are small, so that the quantities of second and higher-order magnitudes may be neglected in comparison with first-order terms in the strain-displacement relations;

3. The component of stress normal to the reference surface is small compared with other normal components of stress;

4. The normals to the undeformed reference surface remain normal to the deformed reference surface and undergo no extension. The first assumption is the basic postulate of the theory. The second assumption, together with Hooke's law, ensures the linearity of the resulting differential equations. The third and fourth assumptions imply the neglect of transverse normal stress and transverse shear deformation. The classical theory of shells (Love's first approximation) is based on these four assumptions. 
Several additional theories have been proposed, in which these basic assumptions are partially or completely modified. A theory which retains second-order terms as compared to unity in the stress resultants and strain-displacements relations, was derived by Flügge (10), Lur'e and Byrne. This improved form of Love's fixst approximation theory has the advantage of removing an inconsistency, of the former; this inconsistency is that, except for the special cases of a spherical shell, a flat plate or a symmetrically loaded shell of revolution, the strains do not all vanish for small rigid-body motions of the shell (see Appendix A). This is the theory we shall use in our analysis.

In the case of a cylindrical shell, the fundamental equations of this theory may be reduced to three coupled linear differential equations relating the three components of the displacement vector, and forming a system of the eighth order; therefore, the solution will have sufficient arbitrariness to satisfy eight boundary conditions.

Because of the cylindrical nature of the system, a cylindrical polar coordinate system $(r, \theta, x)$ will be employed, the origin being taken on the tube axis in the flow inlet cross-section. The shell is allowed radial, longitudinal and circumferential displacements ( $w, u$ and $v$ respectively, w being positive outward); its physical 
properties are defined by the density $\rho_{S}$, Young's modulus $E$ and Poisson's ratio $\nu$. The equations of motion of the shell walls may then be written:

$$
\begin{aligned}
& u^{\prime \prime}+\frac{1-v}{2} u^{\prime \prime}+\frac{1+\nu}{2} v^{\prime}+v w^{\prime}+k\left[\frac{1-v}{2} u^{\prime \prime}-w^{\prime \prime \prime}+\frac{1-v}{2} w^{\prime \cdot \cdot}\right]=8 \frac{\partial^{2} u}{\partial t^{2}} \\
& \frac{1+2}{2} u^{\prime \prime}+v^{\prime \prime}+\frac{1-v}{2} v^{\prime \prime}+w^{\circ}+k\left[\frac{3}{2}(1-v) v^{\prime \prime}-\frac{3-v}{2} w^{\prime \prime}\right]=\gamma \frac{\partial^{2} v}{\partial t^{2}} \\
& v u^{\prime}+v^{\dot{0}}+w+k\left[\frac{1-\nu}{2} u^{\prime \cdots}-u^{\prime \prime \prime}-\frac{3-\nu}{2} v^{\prime \prime}+\nabla^{4} w+2 w^{\prime \prime} w\right]=-\gamma \frac{\partial^{2} w}{\partial t^{2}}
\end{aligned}
$$

where the thickness $\mathrm{h}$ and radius a of the shell are contained in the coefficients $k=h^{2} / 12 a^{2}$ and $\gamma=a^{2} \rho_{s} \frac{1-v^{2}}{E}$. The symbols $(-)$ ' and $(-)$ ' are used for $a \frac{\partial(-)}{\partial x}$ and $\frac{\partial(-)}{\partial \theta}$, respectively.

The boundary conditions are specified on the edges. If the edge is clamped, we may specify that there is neither displacement nor rotation:

$$
u=v=w=0 ; \partial w / \partial x=0
$$

In the case of a free edge, forces and moments may be arbitrarily given. But they are five, $N_{x}, N_{x}, M_{x}, M_{x \theta}$ and $Q_{X}$ whereas we have only four constants of integration. It is usual to replace them by four essential forces and moments which are:

i) the normal force $\mathrm{N}_{\mathrm{X}}=0$

ii) the bending moment $M_{X}=0$, and

iii) Kirchoff's effective shearing stress resultants 
$-14-$

$$
\begin{aligned}
& S_{x}=Q_{x}+\frac{1}{a} \frac{\partial}{\partial \theta} M_{x \theta}=0 \\
& T_{x}=N_{x \theta}-\frac{M_{x \theta}}{a}=0
\end{aligned}
$$

showing the twisting moment $M_{x \theta}$ to be statically equivalent to a tangential shearing force $-\frac{M_{X} \theta}{a}$ and a normal shearing force $\frac{1}{a} \frac{\partial M_{x} \theta}{\partial \theta}$. These two relations may be obtained either by Hamilton's principle or by geometrical considerations.

As the equations of motion relate the three displacement vector components, $u, v$ and $w$, the boundary conditions must also be expressed in terms of these components; this can be done using the following relations:

$$
\begin{aligned}
& N_{x}=\frac{D}{a}\left(u^{\prime}+v v^{*}+v w\right)-\frac{k}{a^{3}} w^{\prime \prime} \\
& N_{\theta}=\frac{D}{a}\left(v+w+2 u^{\prime}\right)+\frac{k}{a^{3}}\left(w+w^{*}\right) \\
& N_{\theta x}=\frac{D}{a} \frac{1-\nu}{2}\left(u^{*}+v^{\prime}\right)+\frac{k}{a^{3}} \frac{1-\nu}{2}\left(u^{*}+w^{\prime *}\right) \\
& N_{x \theta}=\frac{D}{a} \frac{1-\nu}{2}\left(u^{\prime}+v^{\prime}\right)+\frac{k}{a^{3}} \frac{1-v}{2}\left(v^{\prime}-w^{\prime}\right) \\
& M_{x}=\frac{k}{a^{2}}\left(w^{\prime \prime}+\nu w^{\prime \prime}-u^{\prime}-\nu v^{\prime}\right) ; M_{\theta}=\frac{k}{a^{2}}\left(w+w^{\prime}+\nu w^{\prime \prime}\right) \\
& M_{\theta x}=\frac{k}{a^{2}}(1-v)\left(w^{\prime}+\frac{u^{\prime}}{2}-\frac{v^{\prime}}{2}\right) ; M_{x \theta}=\frac{k}{a^{2}}(1-\nu)\left(w^{\prime}-v^{\prime}\right) \\
& Q_{x}=\frac{1}{a}\left(M_{x}^{\prime}+M_{\theta x}^{\circ}\right) \quad ; \quad Q_{\theta}=\frac{1}{a}\left(M_{\theta}^{\prime}+M_{x \theta}^{\prime}\right) \\
& \text { where } D=E h / 1-\nu^{2} \text { and } K=E h^{3} / 12\left(1-\nu^{2}\right)
\end{aligned}
$$

3.2 Natural Frequencies of Clamped-Clamped or ClampedFree Circular Cylindrical Shells

Free vibrations are time-dependent vibratory motions 
set up in the absence of external loads. The free vibration analysis thus deals with a homogeneous system of partial differential equations with homogeneous boundary conditions. These equations and boundary conditions may be written in the symbolic form

$$
\begin{aligned}
& \mathcal{L}_{1}(u, v, w)=\gamma \partial^{2} u / \partial t^{2} \\
& \mathcal{L}_{2}(u, v, w)=\gamma \partial^{2} v / \partial t^{2} \\
& \mathcal{L}_{3}(u, v, w)=\gamma \partial^{2} w / \partial t^{2} \\
& \text { and } \\
& \operatorname{B}_{i}(u, v, w)=0 \quad ; \quad \dot{v}=1,2, \ldots, 8
\end{aligned}
$$

where the $\mathcal{L}_{i}$ and $\beta_{i}$ are differential operators. We may assume that space and time variables are separable and that the displacements may be expressed in the form

$$
\begin{aligned}
& u(\theta, x, t)=u_{0}(\theta, x) e^{i \omega t} \\
& v(\theta, x, t)=v_{0}(\theta, x) e^{i \omega t} \\
& \omega(\theta, x, t)=\omega_{0}(\theta, x) e^{i \omega t}
\end{aligned}
$$

The system now becomes

$$
\begin{aligned}
& \mathcal{L}_{1}\left(u_{0}, v_{0}, w_{0}\right)+\nu \omega^{2} u_{0}=0 \\
& \alpha_{2}\left(u_{0}, v_{0}, w_{0}\right)+\nu \omega^{2} v_{0}=0 \\
& \alpha_{3}\left(u_{0}, v_{0}, w_{0}\right)+\nu \omega^{2} w_{0}=0
\end{aligned}
$$

$$
\text { and }
$$

$B_{i}\left(u_{0}, v_{0}, w_{0}\right)=0 ; i=1,2, \ldots, 8$

and defines a boundary-value problem where the natural frequencies are the eigenvalues and the normal modes of free vibration are the eigenfunctions of the problem. 
The vibrations of a circular cylindrical shell may involve any number, $n$, of waves distributed around the circumference and any number of waves distributed along the length of a generator. The number of axial waves depends upon the end conditions of the cylinder; we shall denote by $m$ the number of axial half-waves.

In the case of homogeneous boundary conditions, the modal frequency will be a function of a single value of $\underline{n}$, and a general solution may be written in the following form:

$$
\begin{aligned}
& u=\sum_{j=1}^{8} A_{j} \exp \left[i\left(\lambda_{j} \frac{x}{a}+n \theta+\omega t\right)\right] \\
& v=\sum_{j=1}^{8} B_{j} \exp \left[i\left(\lambda_{j} \frac{x}{a}+n \theta+\omega t\right)\right] \\
& w=\sum_{j=1}^{8} C_{j} \exp \left[i\left(\lambda_{j} \frac{x}{a}+n \theta+\omega t\right)\right]
\end{aligned}
$$

Substitution of these expressions into the homogeneous differential equations leads to the following eighth-order $\begin{array}{lll}\text { characteristic equation for } \lambda_{j}: & \lambda \\ \lambda^{2}+\frac{1-v}{2} n^{2}(1+k)-\Omega^{2} & \frac{1+\nu}{2} \lambda_{n} & \lambda\end{array}$ $\begin{array}{ccc}\frac{1+\nu}{2} \lambda_{n} & n^{2}+\frac{1-\nu}{2} \lambda^{2}(1+3 k)-\Omega^{2} & n+\frac{3-\nu}{2} \lambda^{2} n k \\ \lambda\left[\nu+k\left(\lambda^{2}-\frac{1-\nu}{2} n^{2}\right)\right] & n+\frac{3-\nu}{2} \lambda^{2} n k & 1+k\left[\left(\lambda^{2}+n^{2}\right)^{2}-2 n^{2}+1\right]-\Omega^{2}\end{array} \mid=C$.

$$
A_{j}=\alpha_{j} C_{j} \quad ; B_{j}=\beta_{j} C_{j}
$$




$$
\begin{aligned}
& \alpha_{j}=\langle-i) \frac{\frac{1+v}{2} \lambda_{n}\left(n+\frac{3-v}{2} \lambda^{2} n k\right)-\lambda\left[n^{2}+\frac{1-\nu}{2} \lambda^{2}(1+3 k)-\Omega^{2}\right]\left[\nu+k\left(\lambda^{2}-\frac{1-\nu}{2} n^{2}\right)\right.}{\left[\lambda^{2}+\frac{1-\nu}{2} n^{2}(1+k)-\Omega^{2}\right]\left[n^{2}+\frac{1-\nu}{2}(1+3 k)-\Omega^{2}\right]-\left(\frac{1+2}{2} \lambda n\right)^{2}} \\
& \beta_{j}=(-i) \frac{\left[\nu+k\left(\lambda^{2}-\frac{1-\nu}{2} n^{2}\right)\right] \frac{1+\nu}{2} \lambda^{2} n-\left[\lambda^{2}+\frac{1-\nu}{2} n^{2}(1+k)-\Omega^{2}\right]\left(n+\frac{3-\nu}{2} \lambda^{2} n k\right.}{\left[\lambda^{2}+\frac{1-\nu}{2} n^{2}(1+k)-\Omega^{2}\right]\left[n^{2}+\frac{1-\nu}{2}(1+3 k)-\Omega^{2}\right]-\left(\frac{1+\nu}{2} \lambda n\right)^{2}} \\
& \Omega^{2}=\gamma \omega^{2}=a^{2} p_{s} \frac{1-\nu^{2}}{E} \omega^{2}
\end{aligned}
$$

into the homogeneous boundary conditions results in eight homogeneous equations in the eight unknown $C_{j} ;$ for nontrivial solution of these equations, the determinant of their coefficients must vanish, and this yields the frequency equation (see Appendix B).

It does not appear feasible to seek analytical expressions for the quantities $\lambda_{j}$. Therefore, at this point in the analysis, a numerical evaluation of the solution is introduced. We now select a given shell (i.e. $a, h, \ell, E, \cdot \nu, \rho_{S}$ are now known), a number of circumferential waves, $n$, and a set of boundary conditions at each end. The method of solution proceeds as follows:

1. An initial estimate for the frequency $\omega$ is taken and upon substitution into the characteristic equation the eight characteristic roots $\lambda_{j}(i=1,2, \ldots, 8)$ are obtained;

2. The assumed frequency $\omega$ and the characteristic roots $\lambda_{j}$ are substituted into the frequency equation; 
3. If the frequency equation is not satisfied, the trial value of the frequency $\omega$ is varied in a systematic way and the procedure is repeated until the frequency equation is satisfied.

For a given wave pattern (fixed numbers of axial half-waves, $m$, and circumferential waves, $n$ ) there exist three natural frequencies, corresponding to different amplitude ratios. The lowest value of $\omega$. which corresponds to predominantly transverse motion of the cylindrical shell, is of greatest interest in our analysis (cf. Kraus (11)p.307). It should also be noted that the number of axial half-waves, $m$, cannot be specified in advance; thus, it is desirable to determine the modal shapelas well as the frequency in each case, since there is an infinite number of frequencies for any fixed value of $n$.

The theoretical results of this analysis will be presented and compared with experimental results in the following chapter.

\subsection{Influence of Fluid Flow}

(a) Pressurization and curvature effects

The influence of fluid flow manifests itself through forces acting upon the inner surface of the shell. These surface loadings per unit area of the reference surface are the longitudinal shear stress $g_{x}$ due to viscous 
drag, and $p_{1}$, the stress due to internal pressure. We shall also take into account the effect of changes of. curvature.

Since we are interested in small perturbations of the shell from its static equilibrium position, we may write the forces and displacements in terms of a steadystate component denoted by the subscript ' $O$ ' and a small time-varying component denoted by the subscript ' 1 '. That is:

$$
\begin{aligned}
u=u_{1} ; v & =v_{1} ; w=w_{0}+w_{1} \\
p & =p_{0}+p_{1}
\end{aligned}
$$

\section{$N_{x}=N_{x_{0}}+N_{x_{1}} ; N_{\theta}=N_{\theta 0}+N_{\theta_{1}} ; \cdot \cdot \cdot \cdot$}

where the fact that $u_{0}=v_{0}=0$ has been utilized. The pressure po is measured with respect to the atmospheric pressure.

$$
\text { Upon substituting these quantities in the }
$$

equations and separating zeroth order terms and first order terms, we obtain the following differential equations relating the time-varying displacements $u_{1}, v_{1}$ and $w_{1}$ (see Appendix C).

$u_{1}^{\prime \prime}+\frac{1-\nu}{2} u_{1}^{\prime}+\frac{1-r v}{2} v_{1}^{\prime \cdot}+\nu w_{1}^{\prime}+k\left[\frac{1-\nu}{2} u_{1}-w_{1}^{\prime \prime \prime}+\frac{1-\nu}{2} w_{1}^{\prime \prime \cdot}\right]-\sigma_{\theta}\left(v_{1}^{\prime-}+w_{1}^{\prime}\right)=\gamma \frac{\partial^{2} u_{1}}{\partial t^{2}}$ 


$$
\begin{gathered}
\frac{1+\nu}{2} u_{1}^{\prime}+v_{1}^{\prime}+\frac{1-\nu}{2} v_{1}^{\prime \prime}+w_{1}^{\prime}+k\left[\frac{3}{2}(1-\nu) v_{1}^{\prime \prime}-\frac{3-\nu}{2} w_{1}^{\prime \prime}\right]+z_{x} v_{1}^{\prime \prime}=\gamma \frac{\partial^{2} v_{1}}{\partial t^{2}} \\
v u_{1}^{\prime}+v_{1}^{\prime}+w_{1}+k\left[\frac{1-\nu}{2} u_{1}^{\prime \cdots}-u_{1}^{\prime \prime}-\frac{3-\nu}{2} v_{1}^{\prime \prime}+\nabla^{4} w_{1}+2 w_{1}+w_{1}\right] \\
-z_{\theta}\left(w_{1}+w_{1}\right)-z_{x} w_{1}^{\prime \prime}-a \mathbb{P}=-\gamma \frac{\partial^{2} w_{1}}{\partial t^{2}}
\end{gathered}
$$

where

$\sigma_{\theta}=\frac{1-\nu^{2}}{E h} N_{\theta 0} ; Z_{x}=\frac{1-\nu^{2}}{E h} N_{x_{0}} ; \mathbb{P}=\frac{1-\nu^{2}}{E h} a p_{1}$ The quantities ${ }_{\theta} \theta$ and $6 x$ are defined by the steady state equations

and

$$
N_{x 0}=q_{x}(l-x)
$$

$$
N_{\theta 0}=a p_{0}(x)
$$

The pressure distribution is found by considering the condition of equilibrium between the shearing stress resultant and the pressure forces

$$
\frac{\partial p_{0}}{\partial x}+\frac{2 q x}{a}=0
$$

Upon integration, and since the outlet pressure is zero, we obtain the pressure of the fluid acting on the shell as a function of the $\mathrm{x}$-coordinate.

$$
p_{0}(x)=\frac{2 q_{x}}{a}(l-x)
$$

We may estimate the viscous shear stress by considering a fully-developed turbulent flow in a pipe. For air flow at 60 feet per second in a $.618 \mathrm{in}$. diameter pipe, the flow 
Reynolds number is

$$
\operatorname{Re}=\frac{V D}{2}=\frac{60 \times 0.618}{1.6 \times 10^{-4} \times 12}=1.93 \times 10^{4}
$$

so that the boundary layer is turbulent. Using experimental data (cf. Schlichting(12) ), we find $q_{x}$ of the order of $2.6 \times 10^{-2} \mathrm{psi}$. Thus for a rubber pipe with characteristics

$$
\nu=0.50 \text { and } E=130 \text { psi }
$$

$\sigma_{x}$ and $\sigma_{\theta}$ are of the order of $10^{-5}$ or less. We may, therefore, neglect the quantities $\sigma_{x}$ and $\zeta_{\theta}$ except in the last equation. At this stage, it is important to note that the steady state tensile forces $\mathrm{N}_{\mathrm{xo}}$ and $\mathrm{N}_{\theta 0}$ are functions of the $\mathrm{x}$-coordinate. This means we have introduced non-constant coefficients in the last differential equation, so that supplementary simplification must later be made, if the method of solution outlined in $\$ 3.2$ is to be used.

\section{(b) Equations of fluid flow}

We must now determine the time-dependent pressure exerted by the fluid on the shell and, therefore, solve the equations of fluid flow. As stated previously, we consider the fluid to be incompressible and the flow to be irrotational so that it may be described by the potential equation:

$$
\nabla^{2} \psi=0
$$


where $\psi$ is the velocity potential. The pressure $p$ is given by Bernoulli 's equation.

Again we introduce steady-state and time-

varying components

$$
\begin{aligned}
\psi & =U_{x}+\phi \\
p & =p_{0}+p_{1} \\
V_{x} & =U+\frac{\partial \phi}{\partial x} \\
V_{\theta} & =\frac{1}{r} \frac{\partial \phi}{\partial \theta} \\
V_{r} & =\frac{\partial \phi}{\partial r}
\end{aligned}
$$

Here $\phi$ is the perturbed potential and $U$ is the free stream velocity in the axial direction under steady conditions. The boundary conditions at the wall of the shell require the matching of the radial velocities of the shell and the fluid.

Assuming a separable solution of the form

$\phi(r, \theta, x, t)=R(r) \exp \left[i\left(\lambda \frac{x}{a}+n \theta+\omega t\right)\right]$

the solution may be expressed in terms of the modified

Bessel function of the first kind, I, and the displacement $w$ (see Appendix $C$ ); the pressure, $p_{1}$, is given by

$$
p_{1}=-\frac{P_{a}}{n+\lambda \frac{I_{n+1}(\lambda)}{I_{n}(\lambda)}}\left[\frac{\partial}{\partial t}+U \frac{\partial}{\partial x}\right]^{2} w
$$

A further refinement involves taking into account the vibrations of the fluid (generally air) surrounding the 
she11; this introduces an additional term involving the modified Bessel function of the second $k i n d, ~ K$, and the displacement $w$, as follows:

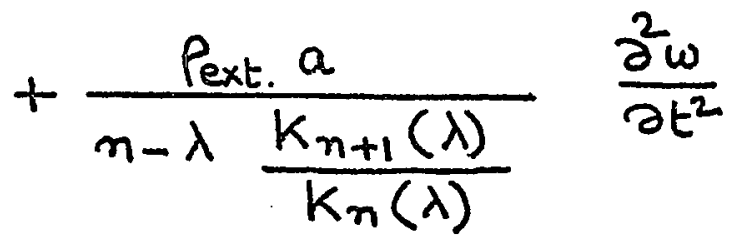

We shall show later that the influence of the fluid surrounding the shell is negligible in comparison with that of the fluid flowing inside the shell.

Before looking for a solution of the system of differential equations, it is of interest to examine the new terms introduced in the equations through the pressure term

$$
p_{1}=-\frac{P a}{n+\lambda \frac{I_{n+1}(\lambda)}{I_{n}(\lambda)}}\left[\frac{\partial^{2} w}{\partial t^{2}}+2 u \frac{\partial^{2} w}{\partial x \partial t}+u^{2} \frac{\partial^{2} w}{\partial x^{2}}\right]
$$

The first term may be identified with the inertia of the fluid in the tube; the factor $\frac{\rho a}{n+\lambda \frac{\operatorname{In+1}(\lambda)}{\ln (\lambda)}}$ then plays the role of a virtual mass per unit surface area for the fluid and is a function of the circumferential wave number, $n$, and the wave length, $\lambda$. If this term is combined with the inertia of the tube $\gamma \frac{\partial^{2} w}{\partial t^{2}}$, a virtual mass may be defined for the fluid-shell system. The third term, involving the second derivative of $w$ with respect to $x$ is 
the centrifugal pressure of the moving fluid. It will tend to increase the curvature of the shell and therefore corresponds to a disturbing force. The midale term, involving the second derivative of w with respect to both $\mathrm{x}$ and $t$, may be identified with the Coriolis force of the moving fluid; it is due to the rotation of the shell and tends to oppose its movement.

\section{4 General solution}

Let us now write the equations of motion, in non-dimensional form and including the terms involving the pressure and the static tensile forces. We employ as the reference for length the radius a, for velocity $\left[E / P_{S}\left(1-2^{2}\right)\right]^{1 / 2}$ and for time $a\left[P_{S}\left(1-2^{2}\right) / E\right]^{1 / 2} \cdot$ Hence we introduce the non-dimensional barred quantities defined as follows:

$\bar{x}=\frac{x}{a} ; \bar{u}=\frac{u_{1}}{a} ; \bar{v}=\frac{v_{1}}{a} ; \bar{w}=\frac{w_{1}}{a} ; \bar{t}=\frac{t}{T_{0}} ; \Omega=\frac{\omega}{\omega_{0}}$ and $\bar{U}=\frac{u}{v_{0}}$

\section{Where}

$$
T_{0}=a\left[\frac{P_{s}\left(1-v^{2}\right)}{E}\right]^{\frac{1}{2}} ; \omega_{0}=\frac{1}{a}\left[\frac{E}{P_{s}\left(1-v^{2}\right)}\right]^{\frac{1}{2}} \text { and } U_{0}=\left[\frac{E}{P_{s}\left(1-v^{2}\right)}\right]^{\frac{1}{2}}
$$

The quantities $\sigma_{\theta}$ and $\bar{\sigma}_{x}$ are already dimensionless since $N_{x o}$ and $N_{\theta 0}$ represent forces per unit length.

The equations may now be written:

$$
\begin{aligned}
\bar{u}^{\prime \prime}+\frac{1-\nu}{2} \bar{u}^{\prime \prime}+\frac{1+\nu}{2} \bar{v}^{\prime \prime} & +2 \bar{w}^{\prime}+k\left[\frac{1-\nu}{2} \bar{u}^{\prime \prime}-\bar{w}^{\prime \prime \prime}+\frac{1-\nu}{2} \bar{w}^{\prime \cdot .}\right] \\
& -2 \bar{\sigma} \times\left(\bar{v}^{\prime \prime}+\bar{w}^{\prime}\right)=\frac{\partial^{2} \bar{u}}{\partial \bar{t}^{2}}
\end{aligned}
$$


$-25-$

$$
\begin{gathered}
\frac{1+v}{2} \bar{u}^{\prime \prime}+\bar{v}^{\prime}+\frac{1-v}{2} \bar{v}^{\prime \prime}+\bar{w}^{\prime}+k\left[\frac{3}{2}(1-v) \bar{v}^{\prime \prime}-\frac{3-\nu}{2} \bar{w}^{\prime \prime}\right] \\
+\bar{\sigma}_{x} \bar{v}^{\prime \prime}=\frac{\partial^{2} \bar{v}}{\partial \bar{t}^{2}} \\
v \bar{u}^{\prime}+\bar{v}^{\prime}+\bar{w}+k\left[\frac{1-v}{2} \bar{u}^{\prime \prime \prime}-\bar{u}^{\prime \prime \prime}-\frac{3-v}{2} \bar{v}^{\prime \prime}+\nabla^{4} \bar{w}+\right. \\
\left.2 \bar{w}^{\prime \prime}+\bar{w}\right]-z_{x}\left(2 \bar{w}^{\prime \prime}+\bar{w}^{\prime \prime}+2 \bar{w}\right)-\mathbb{P}=-\frac{\partial^{2} \bar{w}}{\partial \bar{t}^{2}}
\end{gathered}
$$

with

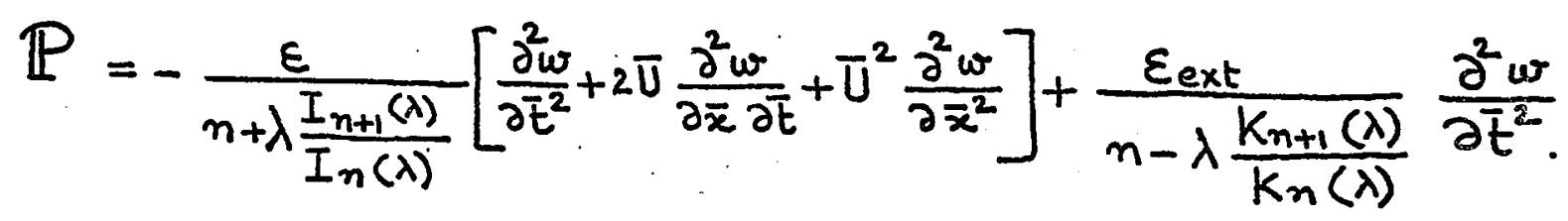

and

$$
\begin{gathered}
(-)^{\prime}=\frac{\partial(-)}{\partial \bar{x}} ;(-)=\frac{\partial(-)}{\partial \theta} \\
\sigma_{\theta}=2 \sigma_{x} ; \varepsilon=\frac{a}{h} \frac{\rho}{P_{s}} ; \quad \varepsilon_{\text {ext. }}=\frac{a}{h} \frac{P_{\text {ext }}}{\rho_{s}}
\end{gathered}
$$

From hereon we shall drop the "bar" symbol specifying the dimensionless quantities.

To proceed with the solution of the equations, it is now necessary to give particular attention to the term ${ }_{6 x}$, since as stated previously it is a linear function of $x$, introducing non-constant coefficients in the equations. We shall take it into account using a smoothing 'technique' replacing it by its average value over the length of the tube, $\int_{0}^{1} 6 x d x$ 
Once again, we look for a solution of the form

$$
\begin{aligned}
& u=\sum A_{j} \exp [i(\lambda x+n \theta+\Omega t)] \\
& v=\sum B_{j} \exp [i(\lambda x+n \theta+\Omega t)] \\
& w=\sum C_{j} \exp [i(\lambda x+n \theta+\Omega t)]
\end{aligned}
$$

which leads to the characteristic equation

$$
\begin{aligned}
& {\left[\lambda^{2}+\frac{1-\nu}{2} n^{2}(1+k)-\Omega^{2}\right] \quad\left(\frac{1+\nu}{2}-2 \sigma_{x}\right) \lambda n \quad \lambda\left[2-2 \sigma_{x}+k\left(\lambda^{2}-\frac{1-\nu}{2} n^{2}\right)\right]} \\
& \frac{1+\nu}{2} \lambda n \quad\left\{n^{2}+\left[\frac{1-\nu}{2}(1+3 k)+\delta_{x}\right] \lambda^{2}-\Omega^{2}\right\} \quad\left(n+\frac{3-\nu}{2} \lambda^{2} n k\right) \\
& \lambda\left[\nu+k\left(\lambda^{2}-\frac{1-\nu}{2} n^{2}\right)\right]\left(n+\frac{3-\nu}{2} \lambda^{2} n k\right)\left\{1-\Omega^{2}+k\left[\left(\lambda^{2}+n^{2}\right)^{2}-2 n^{2}+1\right]+\sigma_{x}\left(2 n^{2}-2+\lambda^{2}\right)\right. \\
& \left.-\frac{\varepsilon(\Omega+U \lambda)^{2}}{n+\lambda \frac{I_{n+1}(\lambda)}{I n(\lambda)}}+\frac{\varepsilon_{\text {ext }} \Omega^{2}}{n-\lambda \frac{K_{n+1}(\lambda)}{K_{n}(\lambda)}}\right\} \text {. }
\end{aligned}
$$

The frequency equation (boundary conditions) is unchanged.

Note - In the case of infinite wave-length, $\lambda=0$, and circumferential wave number $n=1$, corresponding to lateral oscillations, we obtain the following equations :

$A\left[\frac{1-2}{2}(1+k)-\Omega^{2}\right]+B \times 0 .+C \times 0=0$ 


$$
\begin{aligned}
& A \times 0+B\left(1-\Omega^{2}\right)+C=0 \\
& A \times 0+B+C\left[1-\Omega^{2}\left(1-\varepsilon-\varepsilon_{\text {ext }}\right)\right]=0 \\
& \text { The solution is } A=0 \text { and } B=-\frac{C}{1-\Omega^{2}} \simeq-C \text {. } \\
& \text { The displacements are therefore: } \\
& \begin{aligned}
u=0 ; v & =-W \sin \theta \cos \omega t \\
w & =-W \cos \theta \cos \omega t
\end{aligned}
\end{aligned}
$$

The shell vibrates as a beam, which means that the cross-section of the shell remains unchanged in the deformed state. The pressure force per unit length acting on the inside walls of the

$$
\begin{aligned}
& F=\int_{0}^{2 \pi} p_{1} \cos \theta a d \theta=-\rho a^{2}\left(\frac{\partial}{\partial t}+U \frac{\partial}{\partial x}\right)^{2}(\omega \cos \omega t) \int_{0}^{2 \pi} \cos ^{2} \theta d \theta \\
& F=-\rho \pi a^{2}\left(\frac{\partial}{\partial t}+U \frac{\partial}{\partial x}\right)^{2}(W \cos \omega t)
\end{aligned}
$$

This formula shows that the virtual mass of the fluid in these lateral oscillations is

$$
M_{V}=\rho \pi a^{2} \quad \text { per unit length }
$$

which is a well-known result. 


\section{THEORETICAL RESULTS}

Here we are concerned with the study of the dynamics of thin circular cylindrical shells containing flowing fluid. However, before proceeding with this, it is important to first study the free-vibration characteristics of these shells in the absence of flow both to understand the fundamentals of the behaviour of a shell and to test the method which is going to be the basis for the remainder of the analysis. Thus this chapter proceeds with the study of free vibrations first in the absence of flow and then with flow.

\subsection{Free Vibration Characteristics of Clamped-Clamped}

$$
\text { and Clamped-Free Shells in Vacuo }
$$

The method for determining the natural frequencies and modal shapes of circular cylindrical shells for given boundary conditions has been outlined previously. We now proceed to apply it to clamped-clamped and clampedfree sheils, with particular attention to the first case, the study of which is quite detailed.

\section{(a) Clamped-Clamped Cylinders}

The boundary conditions are $u=v=w=0, \frac{\partial w}{\partial x}=0$ at both ends, $x=0$ and $x=l$. For a given set of values of $\mathrm{n}, \mathrm{h} / \mathrm{a}, \mathrm{l} / \mathrm{a}$ and $v$, the determinant $\Delta$ defining the 
characteristic equation yields the eight roots $\lambda_{j}$, $j=1,2, \ldots .8$, which play the role of wave numbers; the determinant $D$ defining the frequency equation yields the natural frequencies.

The characteristic equation may be expressed in terms of the unknown $\lambda^{2}$ and constant, real coefficients; the roots $\lambda_{j}$ are generally complex numbers but may take different forms, i.e.

$$
\begin{aligned}
& \pm(a \pm i b) \text { and } \pm(p \pm i q) \text { for } 0<|\omega|<\omega_{-1} \\
& \pm i a, \pm i b \text { and } \pm(p \pm i q) \text { for } \omega_{-1}<|\omega|<\omega_{0} \\
& \pm i a, \pm b \text { and } \pm(p \pm i q) \text { for }|\omega|>\omega_{0}
\end{aligned}
$$

where $\omega_{-1}$ and $\omega_{0}$ are well-defined values of the circular frequency $\omega$, independent of the boundary conditions, and $a, b, p$ and $q$ are real coefficients. The four values of $\lambda_{j}$ involving the coefficients $a$ and $b$ vary rather rapidly with the circular frequency, $\omega$, whereas the four values of $\lambda_{j}$ involving the coefficients $p$ and $q$ are almost constant with $\omega$. Typical variations with $\omega$ of the first set of roots $\lambda_{j}$ is shown on figure 3 , for a particular tube made of silastic (kind of silicone-rubber material).

Correspondingly, the determinant $\mathrm{D}$ is either real, for $\omega$ less than $\omega_{0}$, or purely imaginary, for $\omega$ larger than $\omega_{0}$, because some roots $\lambda_{j}$ are multiple giving identical columns in the determinant D (see figure 4). These two values $\omega_{-1}$ and $\omega_{0}$ have no physical significance because, once two roots $\lambda_{1}$ and $\lambda_{2}$ are identical, the general solution 
can no longer be given by an expression of the type

$$
A_{1} e^{i \lambda_{1} \frac{x}{a}}+A_{2} e^{i \lambda_{2} \frac{x}{a}}+\sum_{j=3}^{8} A_{j} e^{i \lambda_{j} \frac{x}{a}}
$$

but rather by an expression of the type

$$
\left(A_{1}^{\prime}+A_{2}^{\prime} x\right) e^{i \lambda_{1} \frac{x}{a}}+\sum_{j=3}^{8} A_{j} e^{i \lambda_{j} \frac{x}{a}}
$$

Therefore, the domain of natural frequencies is in the range for which the determinant $D$ is purely imaginary; the first frequency $\mathrm{f}_{1}$ encountered in this domain corresponds to a first axial mode of vibration, $m=1$, as may be checked by determining the corresponding modal shape.

For comparison, the results obtained with this method are brought together with results of other analytical approaches and experiments in figures 5 and 6 . The lowest natural frequencies are plotted versus the circumferential wave-number, $\mathrm{n}$, the parameter being the number of axial half-waves, m. Rayleigh's method $(12)$ is a variational method which assumes an approximate solution satisfying the boundary conditions. "Sanders' theory" is a matricial approach using the theory of Sanders ${ }^{(13)}$. The experimental data were obtained by Koval and Cranch (14) for the results of figure 3 and by a INACA report (15) for results of figure 4 .

We note that the different theoretical results agree closely with one another and with the experimental data, specially in the range where the frequency increases 
with the circumferential wave-number, $n$, that is to say for large $n$. At the lowest values of $n$, the results obtained with the present method show an improvement in agreement with the experimental results.

We next proceed to determine the modal shapes of vibration. To this end we simply solve the eight homogeneous equations with unknowns $c_{j}, j=1,2, \ldots, 8$, corresponding to the eight boundary conditions, and express seven of these unknowns as functions of the eighth one. Typical results are shown in figure 7 ; the radial, longitudinal and circumferential displacements $u, v, w$, respectively, are plotted versus the abscissa $x$ of the shell for the first three modes of vibration. It is of interest to note that the displacement $u$ exhibits one more node than the displacements $v$ and $w$, in each case.

The complexity involved in the use of the shell equations must be tolerated for problems that require knowledge of the free-vibration characteristics of modes having several circumferential waves; in the case of beam-type modes, $\mathrm{n}=1$, on the other hand, considerable simplification may be introduced by considering the cylinder as a compact beam. It is therefore interesting to compare the results obtained by these two approaches: by shell theory and by beam theory. Typical results are shown in figure 8. The agreement is poor for short 
cylinders, the difference between the two results being of order $10 \%$ for a length/radius ratio of 26 ; it seems, therefore, that the extra complexity involved in the use of shell theory is worth the effort for calculating the natural frequencies of thin cylindrical shells vibrating in a beam-type mode. A more detailed comparison of shell and beam theories is given by K. Forsberg ${ }^{(16)}$.

We next examine a point which will be of considerable importance in the subsequent analysis of the vibration characteristics of the cylinder with flow; namely, what is the effect, on the vibration characteristics, of incomplete specification of the boundary conditions? For example, how would the calculated natural frequencies determined by specification of only four boundary conditions differ from those determined precisely by specification of all eight?

As explained previously, the values of $\lambda_{j}, j=1$, $2, \ldots, 8$, may be divided into two sets: four values which take different complex forms, depending on the value of the circular frequency, and four values which keep always the same complex form $\pm(p \pm i q)$. It was found that the magnitude of the real part of these latter $\lambda$ 's is generally ten to twenty times larger than the largest real part of the values of the first set. A typical result is the following: 


$$
\lambda_{j}=\left\{\begin{array}{l}
0.13 \\
-0.13 \\
0.52 i \\
-0.52 i \\
\left\{\begin{array}{l}
+2.11+3.44 i \\
+2.11-3.44 i \\
-2.11+3.44 i
\end{array}\right.
\end{array}\right.
$$

corresponding to the point $\omega_{1}=955$ in figure 4 . Consequently, the harmonics corresponding to the second set of $\lambda_{j}$ have much shorter wavelengths than those corresponding to the first set and their contribution to the modal shape must be small.

It therefore seems reasonable to consider only the first set of values $\lambda_{j}$ and reduce the number of boundary conditions from eight to four, by dropping the conditions involving the longitudinal and circumferential displacements and keeping only those involving the radial displacement. This has been done and the results will now be compared with those of the more general approach. The values of the frequency corresponding to four boundary conditions are somewhat larger than the values corresponding to eight boundary conditions; the difference between the results is shown in percentage form in figure' 9 . The agreement is fairly good for large length/radius ratios, showing that this simplified approach may be used with success 
only for relatively long cylinders.

(b) Clamped-Free Cylinders

The boundary conditions, in this case, specify the displacements at the clamped end:

$$
\mathrm{u}=\mathrm{v}=\mathrm{w}=0 \text { and } \frac{\partial \mathrm{w}}{\partial \mathrm{x}}=0 \text { at } \mathrm{x}=0 \text {, }
$$

and forces and moments at the free end:

$$
\mathrm{N}_{\mathrm{X}}=\mathrm{S}_{\mathrm{X}}=\mathrm{T}_{\mathrm{X}}=0 \text { and } \mathrm{M}_{\mathrm{X}}=0 \text { at } \mathrm{x}=\ell \text {. }
$$

The procedure for calculating the characteristics of free vibration is the same as before.

The characteristic equation is independent of the boundary conditions, so that the particular values of the circular frequencies $\omega_{-1}$ and $\omega_{0}$, are unchanged. As . before, the aeterminant $D$ is either real, for $\omega$ less than $\omega_{0}$, or purely imaginary, for $\omega$ larger than $\omega_{0}$.

The results obtained by this method are compared with those obtained by other analytical approaches in figures 10 and 11. The method of Warburton and Higgs (17) makes use of Flügge's theory and is basically the same as the one we use here; it only differs in that it specifies real displacements $u, v$ and $w$ instead of the more general complex form. "Sanders' theory" is the same matricial method referred to before. We see that the results agree very closely with one another.

Typical results of the modal shapes are shown in figure 12. Again, the shapes are similar to those of beam-type vibration. It is of interest to note that the 
strain in the axial direction, $e_{x}=\frac{\partial u}{\partial x}$ is practically zero at the free end.

The influence of incomplete specification of boundary conditions was also examined in this case, and in particular the possibility of reducing the number of boundary conditions from eight to four. Unfortunately, although it is possible to reduce that number at a clamped end, it is not possible to do the same at a free end and still obtain meaningfull results; no one condition having been found to be preponderant among the four classical ones :

$$
N_{x}=S_{x}=T_{x}=0 \text { and } M_{x}=0
$$

4.2 Vibration Characteristics in the Presence of Fluid Flow

The influence of fluid flow manifests itself through forces coupled to the motions of the shell. These forces act mainly in the radial direction, as seen previously, and correspond to three types of forces: inertia forces, Coriolis forces and centrifugal forces. The apparent mass of the fluid appears to be a function of the wave-number, $\lambda$, through Bessel functions.

At this stage, it is very important to note that, if we follow the method of solution used previously for determining the natural frequencies in the absence of flow, the characteristic equation, being transcendental in $\lambda$, will have an infinite number of roots. This means that the complete solution is no longer a combination of 
eight terms but is now an infinite series. The obvious difficulty lies in the fact that the number of boundary conditions is limited to eight. This method of solution is therefore not well adapted to the problem at hand, unless some further simplification can be made. Fortunately, this is the case. The values of $\lambda$ which contribute most to the modal shapes are those with small modulus; larger real parts correspond to vibrations of shorter wavelength and larger imaginary parts correspond to vibrations with increased damping. In practical terms, the modulus of the roots $\lambda_{j}, j=1,2, \ldots \infty$, increase rapidly with $j$ and the displacements may be adequately represented by the use of a finite series solution, corresponding to a truncated set of $\lambda^{\prime} s$. We shall test the validity of this assumption in the case of clamped-clamped cylinders, in the next section.

\section{(a) Clamped-Clamped Cylinders}

The first case to be studied was that of a clamped-clamped cylinder, as this presented the minimum of numerical difficulties in the computer solution because of the symmetry and simplicity of the boundary conditions. Moreover, this case was flexible in the sense that we could reduce the number of specified boundary conditions from eight to four and thus test the validity of the finite series simplification for the displacements, as referred to above. 
The geometrical and physical characteristics of the cylindrical shell used in the calculations are as follows:

$\begin{array}{ll}\text { length: } & \ell=8.00 \mathrm{in} \\ \text { radius: } & a=0.309 \mathrm{in} \\ \text { thickness: } & \mathrm{h}=0.007 \mathrm{in} \\ \text { shell density: } & \dot{\rho}_{\mathrm{s}} \simeq 3.0810^{-2} \mathrm{lb} / \mathrm{in}^{3} \\ \text { Young's modulus: } & \mathrm{E} \simeq 130 \mathrm{psi} \\ \text { Poisson's ratio: } & \nu \simeq 0.50\end{array}$

They correspond to a rubber tube used in the experiments. The fluid flowing inside the shell is air. As the pressure along the tube is almost constant and equal to the atmospheric pressure, the air density is taken to be

$$
\text { air density: } \rho=0.0765 \mathrm{lb} / \mathrm{ft}^{3}
$$

The fluid outside the shell, and at rest at infinity, is also air.

In the following numerical analysis the coefficient $\mathrm{k}=\mathrm{h}^{2} / 12 \mathrm{a}^{2}\left(\simeq 4 \times 10^{-5}\right.$ ) was neglected in comparison with unity in the characteristic equation; we shall come back to this simplification shortly and question its validity.

Four sets of calculations were performed to study the respective influences of, the specification of the boundary conditions (four or eight boundary conditions), the fluid flow outside the crlinder and the definition of the Bessel functions. 
(i) The first set of calculations was performed using the four-term displacement series corresponding to the four roots $\lambda_{j}$ with the smallest moduli. Accordingly, the boundary conditions are limited to four, namely

$$
w=0 ;-\frac{\partial w}{\partial x}=0 \text { at both } x=0 \text { and } x=\ell
$$

The Bessel functions were approximated by the finite series

$\frac{1}{n+\lambda \frac{I_{n+1}(\lambda)}{I_{n}(\lambda)}}=\frac{1}{2}\left(1-\frac{\lambda^{2}}{12}\right)$ and $\frac{1}{n-\lambda \frac{K_{n+1}(\lambda)}{K_{n}(\lambda)}}=-\frac{1}{2}\left(1-\frac{\lambda^{2}}{4}\right) ;$ for $n=2$

they are coherent with the values of $\lambda$. The results are presented for the first three axial modes of vibrations, $\mathrm{m}=1,2,3$, with the circumferential mode $\mathrm{n}$ equal to 2 , (figure 13).

with increasing flow velocity the frequencies of the first, second and third axial modes decrease with increasing flow velocity (figure 13). They remain real until, at sufficiently high flow velocities, they vanish in turn - at $\overline{\mathrm{U}}=0.58$ and 0.606 in the case of first and second axial modes - indicating the existence of buckling-type instabilities and implying collapse of the shell cross-section. At higher flow velocities, the frequencies become purely imaginary and at $\bar{U}=0.607$ the first and second mode loci coalesce at symmetric points and the frequencies become complex, indicating a coupled- 
mode flutter (figure 14). It is noted that the interval of flow velocity between the onset of static instability (buckling) and flutter is very small; buckling and flutter are practically coincident, and in that sense the theory may be considered to be in qualitative agreement with the observed behaviour, although, of course, strictly speaking the theory ceases being applicable beyond the threshold of the first buckling instability.

(ii) The second set of calculations was performed using the eight-term displacement series corresponding to the eight roots $\lambda_{j}$ with smallest moduli. Accordingly, the eight classical boundary conditions were used,

$$
\mathrm{u}=\mathrm{v}=\mathrm{w}=0 ; \frac{\partial \mathrm{w}}{\partial \mathrm{x}}=0 \text { at both } \mathrm{x}=0 \text { and } \mathrm{x}=\ell
$$

the previous results serving as a guide and points for comparison. The Bessel functions were no longer approximated by second degree polynonials but defined by the full series, where a sufficient number of terms were retained to ensure a good accuracy. Results are presented in figure 13, corresponding to the first two axial modes, $m=1$, 2 , and the second circumferential mode, $\mathrm{n}=2$.

(iii) To evaluate the respective effect of the simplifications concerning the Bessel functions and the boundary conditions, a third set of calculations was performed using the four-term displacement series, the Bessel functions being defined by the full series. Again, the results are presented in figure 13, for the first 
two axial modes $m=1,2$, and second circumferential mode, $\mathrm{n}=2$. We note that the variations in frequency vis-à-vis those of. (i) are very small in all cases; thev are limited to approximately $2 \%$ for zero velocity and are practically nonexistent at buckling velocity. Therefore, it seems that the first approach involving four boundary conditions and simplified Bessel functions is very satisfactory, in particular for the prediction of the buckling instability.

(iv) It is also of interest to study the influence of the fluid outside the cylinder. It should be noted that this fluid has no effect on the buckling velocity, because for buckling the frequency is zero and so is the inertia force which is the only force exerted by the outside fluid on the cylinder. Calculations were carried out assuming vacuum outside the cylinder and using the first simplified approach. The results (figure 13) show a slight increase in frequency for velocities below the buckling velocity and no deviation in the zone of buckling, as expected.

As stated previously, we have neglected the coefficient $k=h^{2} / 12 a^{2}$ in comparison with unity in the characteristic equation. This simplification is apparently valid when the coefficient $k$ is small, as is the case for the preceding calculations where $k$ is equal to $4 \times 10^{-5}$, but in fact the magnitude of all the terms should be examined to decide whether this simplification is valid or not. 
The dimensionless frequency, $\Omega$, is of order $2 \times 10^{-2}$, the circumferential wave number is equal to 2 , the roots $\lambda_{j}$ have a modulus of order $2 \times 10^{-1}$ and Poisson's ratio $\nu$ is equal to 0.5. Thus the terms involving the coefficient $k$ are of the same order as $\Omega^{2}$ and, therefore, the coefficient $k$ should not be neglected. Only for thinner shells, where $k$ is small in comparison with $\Omega^{2}$, may it be neglected. A new set of results was obtained this time without any simplification in the characteristic equation and using four boundary conditions and the full Bessel functions. The results are compared with the previous ones for the first three axial modes in figure 13. They show an increase in frequency of the order of $5 \%$ for zero velocity, the buckling velocity being also slightly increased. Accordingly, although the effect of neglecting $k$ in comparison with unity is small, it should not be disregarded. The previous results are thus slightly affected quantitatively but can still be relied upon. In the following calculations no simplification will be introduced in the full characteristic and frequency equations and the full Bessel functions will be used.

Some further calculations for $n$ other than $n=2$ have been conducted and the results are shown in figure 15. . The next step in this analysis was to study the variations in instability thresholds with the length of the cylinder; more precisely, the onset of buckling instability was considered because it can be predicted by the present theory as a limiting case and it corresponds to simple calculations. Four boundary conditions are 
$-42-$

used in these calculations. The dimensionless velocity corresponding to buckling is plotted versus the length/ radius ratio for the first two or three axial modes and the first, second and third circumferential wave-numbers (figure 16). It is noted that the curves tend to asympLotic values for large length/radius ratios. For $\mathrm{n}=2$ and $\mathrm{n}=3$, there is an "exchange" of loci between the axial modes when two curves happen to cross each other; so that the lower portions of curves always correspond to first axial modes, the following upper to second axial modes, then third.

For the case $\mathrm{n}=1$, the numerical analysis is particularly simple as an explicit expression for the velocity corresponding to buckling may be obtained. In the case of buckling, the roots $\lambda_{j}, j=1,2,3,4$, are simply

$$
\lambda_{1}=-\lambda_{2} ; \lambda_{3}=\lambda_{4}=0
$$

Therefore, the solution takes the limiting form

$$
w=A+B x+C \cos \lambda_{1} \cdot \frac{x}{a}+D \sin \lambda_{1} \frac{x}{a}
$$

Application of the boundary conditions

$$
w(0)=w(l)=0 \text { and } \frac{\partial w}{\partial x}(0)=\frac{\partial w}{\partial x}(l)=0
$$

leads to the equation

$\left(\sin \frac{\lambda_{1} l}{2 a}-\frac{\lambda_{1} l}{2 a} \cos \frac{\lambda_{1} l}{2 a}\right) \sin \frac{\lambda_{1} l}{2 a}=0$ 
the solution of which if formed by the two sets of roots

$$
\frac{\lambda_{1} l}{2 a}=p \pi ; p=1,2, \ldots \text { and } \frac{\lambda_{1} l}{2 a}=4.4934, \ldots
$$

The value $\frac{\lambda_{1} l}{2 \bar{a}}=\pi$ corresponds to the first axial mode, the value $\lambda_{1} l=4.4934$ corresponds to the second axial mode. The explicit expression for the velocity is then obtained from the characteristic equation

$$
\begin{aligned}
& \lambda^{2}+\frac{1-\nu}{2}(1+k) \quad \frac{1+\nu}{2} \lambda \quad \lambda\left[\nu+k\left(\lambda^{2}-\frac{1-\nu}{2}\right)\right] \\
& \frac{1+\nu}{2} \lambda \quad 1+\frac{1-2}{2} \lambda^{2}(1+3 k) \quad 1+\frac{3-2}{2} \lambda^{2} k \\
& \lambda\left[\nu+k\left(\lambda^{2}-\frac{1-\nu}{2}\right)\right] \quad 1+\frac{3-\nu}{2} \lambda^{2} k \quad 1+k\left[\left(\lambda^{2}+1\right)^{2}-1\right]-\frac{\varepsilon}{1+\lambda \frac{I_{2}(\lambda)}{I_{1}(\lambda)} \nu^{2} \lambda^{2}} \\
& \text { where the fact that } \Omega=0 \text { and } n=1 \text { has been taken into } \\
& \text { account. Returning now to the examination of figure } 16 \text {, } \\
& \text { the results show that for a short cylinder, the first } \\
& \text { instability occurs in the second circumferential mode, } \\
& \text { whereas above a certain length the first instability occurs } \\
& \text { in the first circumferential mode. These results will } \\
& \text { be compared with experimental ones. Finally, another } \\
& \text { point of interest is the variation of modal shapes with } \\
& \text { flow velocity. The method of calculation and associated } \\
& \text { analysis are presented in Appendix D. The results are } \\
& \text { presented in figures } 17 \mathrm{a}, \mathrm{b}, \mathrm{c}, \mathrm{d}, \mathrm{e} \text { and } 18 \mathrm{a}, \mathrm{b}, \mathrm{c}, \mathrm{d} \text {, e } \\
& \text { and correspond to } \mathrm{m}=1 \text { and } 2 \text {, and } \mathrm{n}=2 \text { throughout, for } \\
& \text { different velocities ranging from zero to buckling velocity; }
\end{aligned}
$$


each figure shows the time-dependence over half a period of the modal shapes in terms of $u(x, \theta, t), v(x, \theta, t)$ and $w(x, \theta, t)$. It is noted that the modes change their form with increasing velocity, the first mode at zero velocity exhibits a second mode shape just before buckling and the second mode at zero velocity exhibits a third mode shape just before buckling.

(b) Clamped-Free Cylinders

For this particular set of boundary conditions a single approach was used. The solution was assumed to be an eight-term series, coherent with the eight classical boundary conditions,

$$
\begin{aligned}
& \mathrm{u}=\mathrm{v}=\mathrm{w}=0, \frac{\partial \mathrm{w}}{\partial \mathrm{x}}=0 \text { at one end } \mathrm{x}=0 \\
& \mathrm{~N}_{\mathrm{X}}=\mathrm{S}_{\mathrm{X}}=\mathrm{T}_{\mathrm{X}}=0, \mathrm{M}_{\mathrm{x}}=0 \text { at the other end } \mathrm{x}=\ell .
\end{aligned}
$$

The influence of fluid inside and outside the shell was taken into account, using the full expressions for the Bessel functions $I$ and $k$. No simplification was introduced in the characteristic and frequency equations.

The geometrical and physical characteristics of the cylindrical shell used in the calculations were the same as before. Calculations were done for the first two axial modes and for circumferential modes equal to 1, 2 and 3. The results are shown in figure 19; they present the variations of the dimensionless frequency in the frequency-plane (Argand diagram) with the parameter 
$\bar{U}$, i.e. the dimensionless flow velocity.

It is noted that the effect of flow for small

values of $\bar{U}$ is to damp the system in all modes; the. frequencies become complex with positive imaginary parts. For higher values of $\bar{U}$, however, some of the modes (the second axial modes) become less damped and the corresponding frequency curves eventually cross the real axis, at points where the real parts are finite, proving the existence of unstable vibrations. Some other modes (the first axial modes), on the contrary, become more damped with flow and the corresponding frequency curves reach the imaginary axis, at points where the imaginary parts are finite and positive, so that motion in these modes then becomes completely non-oscillatory. The second axial modes become unstable before the first axial modes are damped without oscillations. The instability associated with the second axial modes correspond to flexural (beamtype) oscillations for $\mathrm{n}=1$ and to flutter for $\mathrm{n}=2$ and $\mathrm{n}=3$.

It is noted that these results are qualitatively similar to those presented by R.W. Gregory and M.P. Paidoussis in the simple case of flexural oscillations $(n=1)$.

Next, the influence of the length of the cylinder on the onset of instability was considered. Results presented in figure 20 show the variations of the critical velocities with the length/radius ratio, for the second axial modes corresponding to $\mathrm{B}=1,2$ and 3 . The system 
first becomes unstable in its second mode, $n=2$, for short cylinders; for long cylinders, it first becomes unstable in its first mode, $\mathrm{n}=1$, as would be expected.

(c) Physical Interpretation of the Theoretical Results

At this point in the analysis, it is interesting to question the results just obtained and try to give a physical interpretation of the phenomenon. In particular, is there a simple argument to anticipate the theoretical results just derived and explain the differences between a system with clamped-clamped tube and a system with clamped-free tube?

The basic difference between these two systems is that the first one, with symmetrical clamped end conditions, is conservative whereas the second, with asymmetrical clamped-free end conditions is not. By conservative we mean that during a continuous vibration the transfer of energy between fluid and tube must have a zero average, or in other words, that the average supply of energy at inlet is the same as the average loss at outlet.

It is interesting to define the energy accumulated or rejected by the overall system, i.e. the pipe and the fluid enclosed in it. Benjamin (2) considered this mechanism of energy transfer in the problem of articulated pipes conveying fluid; in a motion over a time 0 to $t_{1}$ which concludes with the system in its original state, if 
the upstream end is clamped or simply supported, the energy gained by the pipes is

$\Delta W=-\int_{0}^{t_{1}} M U\left(\dot{R}^{2}+U \vec{\tau} \cdot \overrightarrow{\dot{R}}\right) d t \quad ; \quad \vec{R}=\frac{d \vec{R}}{d t}$

where $M$ is the mass per unit length of the contained fluid and $\vec{C}$ and $\vec{R}$ are the tangential and position vectors at the end of the last pipe. Let us recall the hypotheses which justify this formula:

(i) The system considered has a finite number of degrees of freedom.

(ii) A set of Lagrangian equations is found in terms of the energies of the finite part of the overall system, i.e. the part comprising the pipes and the enclosed volume of fluid, and condensed into an appropriate statement of Hamilton's principle which remains correct in the case of infinite freedom.

(iii) The kinetic energy of the assembly of particles momentarily filling the pipes is expressed as:

$$
T^{\prime}=T 1+T 2
$$

where $\mathrm{Tl}$ is the kinetic energy contained at any time within the space enclosed by the pipes and the second term $\mathrm{T} 2$ is the time-dependent kinetic energy, correct to first order of $\left(t-t_{0}\right)$.

It is important to note that the energy transfer cannot be related to the time rate of change of some 'total energy' of the finite system, owing to the fact 
that the time variable $t$ is explicitly involved. Physically, the energy $\Delta W$ is the rate of work by the non-conservative part of the hydrodynamic forces; the first term MU: ${ }^{2}$ represents the average work done by the pipes against Coriolis reactions of the fluid, and the second term $\mathrm{MU}^{2} \vec{\tau} \cdot \overrightarrow{\mathrm{R}} \quad$ represents the average work done by the pipes against the 'equivalent force' of the relative nomentum flux $M U^{2}$ out of the last pipe.

So far we have assumed that the upstream end is clamped or simply supported, but we have not introduced the boundary condition at the downstream end. If this end is also clamped or simply supported, we have $\Delta W=0$. This means that vibrations can neither be damped nor amplified by the action of the flow; the hydrodynamic forces are then of purely conservative type. In terms of the complex frequency diagrams, the frequency of all the modes remains real with increasing velocity and follows the $[\operatorname{Re}(\Omega)]$ - axis toward the origin. Thus, in the absence of frictional forces, the only possible form of instability is buckling. At the origin, the frequency locus bifurcates and the two branches continue along the positive and negative $[\operatorname{Im}(\Omega)]$ - axis. Thus, once buckling occurs, the centrifugal force causes amplification of the deformation. Beyond that point, the theory ceases being applicable, as additional non-linear. forces will come into effect. However, we may hope that the qualitative prediction of this theory is still right after 
buckling. With a further increase of velocity, the first and second axial mode loci coalesce at symmetric points, and the frequencies become complex, indicating a coupledmode flutter; in practical terms the interval of flow velocity between static instability (buckling) and flutter is very small and the theory may be considered to be in qualitative agreement with the observed behaviour. If the downstream end is free, the hydrodynamic forces are of non-conservative type. When the velocity $U$ is small, vibrations are damped since the first term in the integrand predominates over the second and makes $\Delta W$ negative. For sufficiently high velocity U, amplified vibrations are possible $(\Delta W>0)$ provided the scalar product $\vec{\tau} \cdot \vec{R}$ has a negative average value; this means that for the greater part of a cycle the downstream end of the pipe must slope backwards to the direction of motion of its free end and perform a 'dragging' sort of motion. This 'dragging' motion is obtained with the second and fourth axial modes and indeed the theoretical results show that, at a sufficiently high velocity, the amplitude of vibration of the second axial mode is amplified whereas those of the first and third axial modes are damped, whatever the circumferential mode may be. In this case, oscillatory instabilities are possible, independently of frictional forces.

These general remarks provide a clear physical interpretation of the effects of the fluid upon the pipe. 
Two different forms of instability are in evidence; one is termed 'buckling' and the other is self-excited vibrations. 


\section{CHAPTER V}

\section{EXPERIMENTS}

\subsection{Introduction.}

The experimental work described here was supplementary to the theoretical study. The aim was twofold: first, to study the dynamical behaviour of flexible cylinders conveying fluid and confirm some of the leading ideas explained in the theoretical part; secondly, to measure the limits of stability in a number of cases, and compare them with the theoretical values. The experiments were conducted with circular cylinders of mean radius $a$, thickness $h$, length $\ell$, density $\rho_{S}$ and flexural rigidity EI, in a fluid of density $\rho$ flowing with velocity $U$ parallel to the rest position of the cylinder axis. One end of the cylinder was clamped, i.e. tied to a rigid tube, the other was either clamped or free. The cylinder was hanging down. No tension was applied externally. For comparison of the experimental with the theoretical results, the following dimensionless parameters were used:

$$
\begin{aligned}
& \text { dimensionless frequency } \Omega=\frac{\omega}{\omega_{0}} \text { with } \omega_{0}=\frac{1}{a}\left[\frac{E}{\rho_{S}\left(1-v^{L}\right)}\right]^{1 / 2} \\
& \text { dimensionless flow velocity } \bar{U}=\frac{U}{U_{0}} \text { with } U_{0}=\left[\frac{E}{\rho_{S}\left(1-v^{2}\right)}\right]^{1 / 2}
\end{aligned}
$$

\subsection{Apparatus}


made of "silastic" or rubber. "Silastic" is a silicone rubber with physical characteristics defined by a Young's modulus, $E \simeq 215$ psi, a Poisson's ratio, $v \simeq 0.47$, and a density, $\rho_{S} \simeq 3.72 \times 10^{-2} \mathrm{lb} / \mathrm{in}^{3}$; these cylinders were cast in specially prepared moulds. The rubber tubes were made of latex rubber colostomy tubing; this material is characterized by $\mathrm{E} \simeq 130 \mathrm{psi}, \nu \simeq 0.50$ and $\rho_{\mathrm{S}} \simeq 3.08 \mathrm{x}$ $10^{-2} \mathrm{lb} / \mathrm{in}^{3}$. Typical dimensions of the tubes are given by the following data:

$$
\begin{aligned}
& \text { Silastic tubes } \begin{cases}\text { mean radius, } a=0.28 \text { in } \\
\text { thickness }, \mathrm{h}=0.06 \text { in } \\
\text { length }, \ell=2 \text { in to } 10 \text { in }\end{cases} \\
& \text { Rubber tubes } \begin{cases}\text { mean radius, } a=0.309 \text { in } \\
\text { thickness., }, \quad=0.007 \text { in } \\
\text { length }, \ell=1 \text { in to } 10 \text { in }\end{cases}
\end{aligned}
$$

For almost all the experiments air was used as the fluid; some observations were made when the fluid was water. The apparatus consisted mainly of an air-compressor, flow-rators to measure the rate of air-flow, valves, and connexions to fix the flexible tubes. For observation of the vibrating tubes and measurement of frequencies a stroboscope was used; it could be synchronized by any external periodic electrical signal; in the experiments this signal was given either by a variable frequency generator or transmitted by a microphone recording the sound produced by the vibrating tubes. This set-up allowed 
"freezing" or slow-motion observation of the tubes. A schematic diagram of the experimental apparatus is presented in figure 21 .

5.3 General Observations

We first consider the clamped-free cylinders, because in this case, the downstream end of the cylinder is free and the deformations of its cross-section are directly observable from above, thus providing a simple way of determining the circumferential mode of vibration. In the experimental procedure the air-flow velocity is increased in small steps, starting from zero.

For a velocity below the flutter threshold, the first vibrations to appear correspond to the second circumferential mode. The amplitude of these vibrations is small, the end cross-section takes a quasi-elliptical shape and deforms alternately between two extreme positions. With increasing velocity the amplitude of these vibrations increases slightly until at a certain threshold the vibrations change and correspond then to a different circumferential mode. This mode corresponds to $n=4$ for a rubber tube; for a silastic'tube, it is possible to observe vibrations corresponding to $\mathrm{n}=3$. It should be noted that the sound of these vibrations is low and cannot be easily recorded.

Increasing the velocity further, the flutter threshold is reached. Vibrations of large amplitude appear 
in the region near the free end and are of diminished amplitude toward the clamped end. The shape of the free-end cross-section is irregular and becomes wobbly with increasing velocity: its lips twist alternately outward and inward and close the opening almost completely at the two extremes of the cycle. The cross-section of the free end exhibits a shape corresponding to the second circumferential mode of the shell with no flow (see photographs). Reducing the flow velocity, flutter persists below the critical flow.

The frequency of vibrations ranges between $100 \mathrm{~Hz}$ and $800 \mathrm{~Hz}$ and increases with increasing flow velocity. The noise level is high; typical principal frequencies of the emitted sound, recorded with a microphone, are presented below for a silastic tube. 


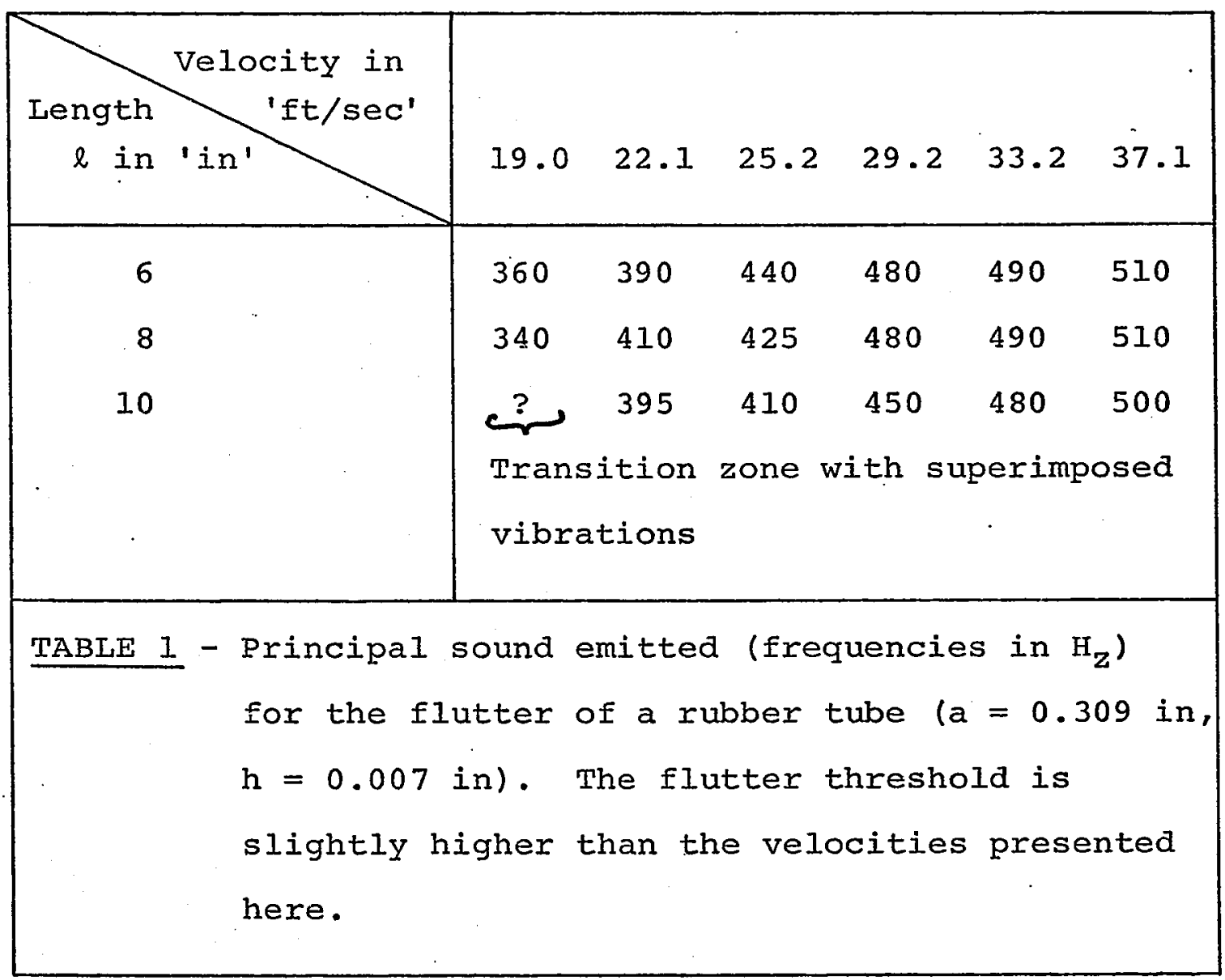


In the case of relatively thick shells (silastic tubes in particular) and at high velocities, internal stresses cause heating of the material and eventually rupture of the shell near the downstream end.

Flutter develops spontaneously but it may also be induced at velocities lower than the critical by pinching or disturbing the tube.

With a cylinder clamped at both ends, the characteristics of flutter are the same as for a clampedfree cylinder. For short cylinders, a flexural buckling ( $\mathrm{n}=1)$ appears before the flutter. The vibrations in this case also, appear in the region near the downstream end.

5.4 Measurements of the Flutter Thresholds

The quantitative tests comprise measuring the air flow-velocity at which flutter occurs, and comparing the theoretical and experimental values. 


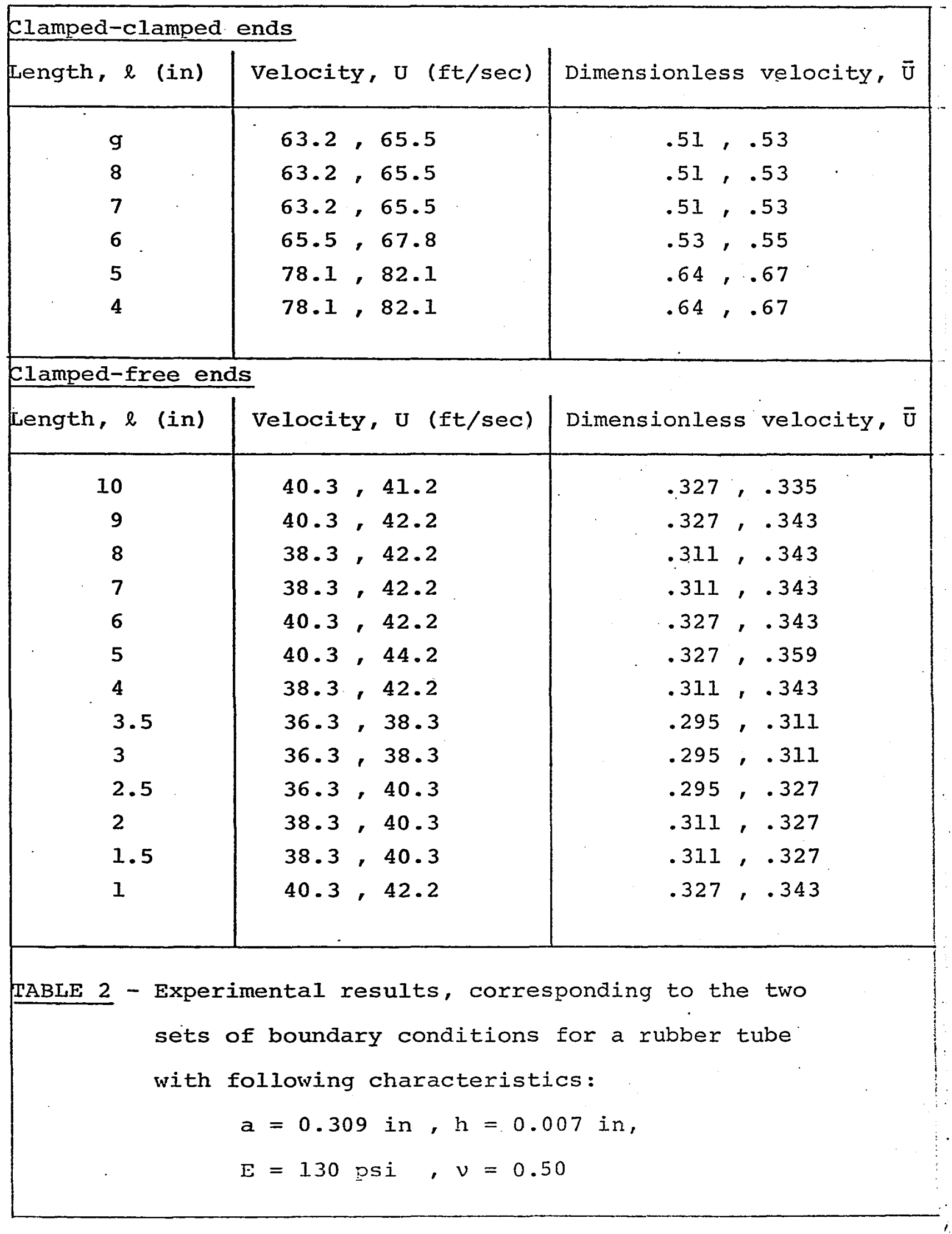


Comparison of these results with theoretical ones is presented in figures 16 and 20 .

In the clamped-clamped case, the theoretical results correspond to the threshold of buckling instabilities; this is admissible, however, as we have shown that buckling and flutter instabilities are practically coincident. The general agreement between theory and experiment is good. As expected, the experimental results lie below the theoretical ones; the margin of discrepancy varies between 0 and $16 \%$. The divergence may be due to secondary effects such as smallirregularities of the tube and the clamped ends.

In the clamped-free case, the theoretical results are the instabilities of the second axial modes as we have shown that they are the first to occur. The agreement between theory and experiment is rather good for long tubes ( $\ell$ larger than 5 in, i.e. $\ell / a>16$ ) where the margin is about $20 \%$. For shorter tubes however, $(l / a<16)$, the divergence is more important, it may be due again to irregularities of the tube and the clamped end; moreover, secondary flows at the outlet such as vortices and eddies may also cause or, at least, affect the onset of, instabilities. Experiments were carried out with smoke instead of air, but no specific pattern or motion of the smoke could be seen at the free end. rie do not here present any experimental results concerning the frequencies of vibration of the shell. Such experiments. vere, hovever, carried 
out with a rubber tube. Before flutter occurs, the tube vibrates in the fourth circumferential mode $(n=4)$ with very small amplitude, whereas in the present study, theoretical results are given only for $n=1,2,3$. The frequencies are in the range $(100 \mathrm{~Hz}, 120 \mathrm{~Hz})$ and should correspond to the theoretical values for $n=4$. After flutter occurs, it is difficult to measure the frequencies of vibrations, simply because several frequency components are present - except at very low velocities where the second circumferential mode is predominant.

Although the apparatus was of the simplest kind, the experiments appeared to confirm the essential features of the dynamical problem predicted by the theory. On the whole, the comparison between theory and experiment is reasonably favourable. 


\section{CHAPTER VI}

\section{CONCLUSION}

The existence of unstable vibrations of circular cylindrical shells conveying fluid has been established and the conditions of stability have been determined for some possible physical systems.

Discussion of the stability of equilibrium has illuminated the remarkable and basic difference between a system with clamped-clamped ends and one with clamped-free ends; it lies in the fact that the hydrodynamic forces on the tube are conservative when both ends are clamped, whereas they are non-conservative when one end is free. This important property explains in particular the two different forms of possible instability. In the case of a tube with clamped-clamped ends, buckling instability appears first, as the system is of conservative type; beyond buckling, coupling between the first two axial modes of vibration is predicted, giving rise to a coupledmode flutter. In the case of a tube with clamped-free ends, instability consists of self-excited vibrations, as the system is then of non-conservative type; in agreement with the conciusions drawn from the study of the stability of equilibrium, the system has been shown to become unstable first in its second axial mode, the tube performing then a "dragging" sort of motion.

The conditions of stability have been letermined for different values of the laneth/racius ratio, as this is 
the most significant physical parameter. For short tubes, and whatever the end conditions may be, the system becomes unstable first in its second circumferential mode, either by buckling for clamped-clamped end conditions or self-excited vibrations for clamped-free end conditions; for longer tubes, it first becomes unstable in its first circumferential mode, i.e. flexural or beam-type buckling or vibrations; this behaviour is to be expected. The system is also more easily destabilized with increasing length/radius ratio.

Another interesting property of the system under consideration is the exchange of stability loci between axial modes with varying length/radius ratio, in the case of clamped-clamped tubes. At certain points different curves corresponding to buckling in different axial modes (figure 16) converge and cross each other; this means that buckling and coupling between modes are then coincident and flutter is expected to happen with buckling.

In connection with the phenomenon of flow-induced buckling, a complementary, simple method of approach was used in the case of beam-type buckling $(n=1)$ where the question of stability was conceived as a statical problem (page 42), the effects of the flow being then considered equivalent to the centrifugal force of the moving fluid. This simple approach gives imnediate insight into the physical mechanism of buctiling and shous directy 
the forces acting on the system. However, this approach cannot be extended to the general case, where a dynamic theory must be used. Moreover, it should be noted that a statical theory cannot predict the form of the equivalent mass of fluid acting on the walls of the pipe, which in our case is deduced from potential flow theory.

The more interesting results of the general theory are those which illustrate the role of the fluid as a source or sink of energy, just as the more interesting experimental observations are concerned with this aspect. The experiments, although conducted with apparatus of a simple kind, appeared to confirm the essential features of the dynamical problem predicted by the theory. strictly speaking, it cannot be expected that the behaviour of the system with increasing flow velocity can be predicted by the linear analysis beyond the point where instability first occurs; the amplitude of motion then grows to the extent that forces not considered in the analysis come into play: It was particularly interesting, therefore, that the predicted coupled-mode flutter instability for clamped-clamped tube materialized as the flow velocity increased beyond the point where buckling occurred. In this case, and for relatively short tubes, buckling and flutter instabilities in the second circumferential mode were practically coincident; for longer tubes, the first instability to occur was buckling in the first circumferential mode, i.e. bean-type buckling, followed with 
increasing flow velocity, by flutter instability, either with $\mathrm{n}=1$ or $\mathrm{n}=2$ or even both of them. In the case of clamped-free tubes, self-excited flutter appeared; . depending on the length of the tube, the modes $n=1$ or $\mathrm{n}=2$ would appear separately, the mode $\mathrm{n}=3$ could also be excited with particular initial conditions.

As mentioned in the introduction, these particular vibrations of an elastic tube had never been reported or studied hitherto. The present paper provides a description and theoretical investigation of this problem. Although the proposed theory is involved and deals with cumbersome equations, the key to understanding the underlying physical mechanism remains the simple consideration of the stability of equilibrium whereby the basic difference between systems with clamped-clamped and clamped-free end conditions is introduced; this allows distinction between conservative and non-conservative systems and proves to be greatly enlightening as regards the physical side to the problem.

Undoubtedly, this problem is worth supplementary investigations. The influence of the thickness/radius ratio could first be studied; this should be straightforward, as most of the results can be predicted. Similarly, the influence of the nature of the fluid could be studied; in particular, with water the nature of instabilities is different and it would be interesting to examine the reasons for this behaviour. Also, and though it is perhaps a slight digres- 
sion from the main problem, it would be interesting to study the flow patterns and possible vortex generation at the free end of a tube and see its perturbing influence on the stability of a clamped-free tube. Finally, in connection with possible applications of this problem, none is anticipated so far, but no doubt some will show up in the future. This study was aroused merely by scientific curiosity and its aim was simply to obtain a deeper understanding and knowledge in the mechanics of an intriguing physical phenomenon. 


\section{REFERENCES}

(I) NIORDSON, F.I.N.

'Vibrations of a Cylindrical Tube Containing Flowing Fluid'

K. Tek. Hogsk. Handl., 1953, No. 73.

(2) BENJAMIN, T.B.

'Dynamics of Articulated Pipes Conveying Fluid'

Proc. ROY. Soc., 1961, A, Voi. 261.

(3) a- PAIDOUSSIS, M.P.

'Dynamics of Tubulax Cantilevers Conveying Fluid'

J. Mech. Eng. Science, 1970, Vol. 12.

b- GREGORY, R.W. and PAIDOUSSIS, M.P.

'Unstable Oscillation of Tubular Cantilevers

Conveying Fluid - I. Theory'

Proc. Roy. Soc., 1966, A, Vol. 293.

(4) BOLOTIN, V.V.

'Non-Conservative Problems of the Theory of

Elastic Stability'

1963, MacMillan Company: New York.

(5) MILES, J.W.

'Supersonic Flutter of a Cylindrical Shell'

J. Aero. Science, 1957, Vol. 24.

(6) a- DOWELL, E.H. and WIDNALI, S.E.

'Generalized Aerodynamic Forces on an Oscillating Cylindrical Shell'

Quart. of Appl. Math., 1966, Vol. 24, No. 1.

b- DOWELL, E.H. and WIDNALL, S.E.

Generalized Aerodynamic Forces on an Oscillating Cylindrical shell: Subsonic and Supersonic Flow'

AIAA Journal, 1966, Vol. 4, No. 4.

(7) KUDRYAVTSEV, E.P.

'On the Flutter of Elastic Cylindrical Coaxial

Shells Between Which Flows a Compressible Fluid'

Theory of Shells and Plates. Academy of Sciences

of the Armenian S.S.R., Israel Program for Scientific

Translations, 1966.

(8) TIMOSHENKO, $\mathrm{S}$.

'Theory of Plates and Shells'

First Edition 1940, McGraw-Hill: New York.

(9) LOVE, A.E.H.

'The Mathematical Theory of Elasticity'

Fourth Edition 1927, Cambridge University Press. 
(10) FLÜGGE, W.

'Statik und Dynamik der Schalen'

Second Edition 1957, Springer: Berlin.

(11) KRAUS, H.

'Thin Elastic Shells'

1967, John Wiley \& Sons.

(12) SCHLICHTING, $H$.

'Boundary Layer Theory'

Fourth Edition 1960, MCGraw-Hill.

(13) a- SANDERS, J.I., JR.

'An Improved First-Approximation Theory for Thin Shells'

NASA Technical Report R-24, 1959.

b- LAKIS, A.A.

'Free Vibration and Response to Random Pressure

Field of Non-Uniform Cylindrical Shells'

Ph.D. Thesis, March 1971, Dept. of Mech. Eng.,

McGill Univ., Montreal.

(14) KOVAL, L.R. and CRANCH, E.T.

'On the Free Vibrations of Thin Cylinarical Shells.

Subjected to an Initial Static Torque'

Proc. 4th U.S. Nat. Congr. Appl. Mech., 1962.

(15) SEWALI, J.L. and NAUMANN, E.C.

'An Experimental and Analytical Vibration Study of Thin Cylindrical Shells With and without

Iongitudinal Stiffeners'

NASA TN D $-4705,1968$.

(16) FORSBERG, K.

'Axisymmetric and Beam-Type Vibrations of Thin

Cylindrical Shells'

AIAA Journal, 1969, Vol. 7, No. 2 .

(17) WARBURTON, G.B. and HIGGS, J.

'Natural Frequencies of Thin Cantilever Cylindrical Shells'

J. Sound Vib., 1970, Vol. 11 (3).

(18) WATSON, G.N.

'A Treatise on the Theory of Bessel Functions'

Second Edition 1966, Cambridge University Press. 
OTHER REFERENCES

WARBURTON, G.B.

'Vibration of Thin Cylindrical Shells'

J. Mech. Eng., 1965, Vol. 7, No. 4 .

WARBURTON, G.B. and AI-NAJAFI, A.M.

'Free Vibration of Thin Cylindrical shells with

a Discontinuity in the Thickness'

J. Sound Vib., 1969, Vol. 9 (3).

FORSBERG, K.

'Influence of Boundary Conditions on the Modal

Characteristics of Thin Cylindrical Shells'

AIAA Journal, 1964, Vol. 2, No. 12 .

FORSBERG, $\mathrm{K}$. ,

'A Review of Analytical Methods Used to determine the Modal Characteristics of Cylindrical Shells'

NASA, 1966 , CR. 613.

PAIDOUSSIS, M.P.

'Dynamics of Flexible Slender Cylinders in Axial Flow'

J. Fluid Mech., 1966, Vol. 26, Part 4.

NAGHDI, P.M. and BERRY, J.G.

'On The Equations of Motion of Cylindrical Shells'

J. Appl. Mech., 1954, Vol. 21, No. 2 .

KORNECKI, A.

'A Note on Beam-Type Vibrations of Circular

Cylindrical shells'

J. Sound Vib., 1971, Vol. 14 (1).

YI-YUAN . YU

'Free Vibrations of Thin Cylindrical shells Having

Finite Lengths With Freely supported and Clamped

Edges'

J. App1. Mech., 1955, Vol. 22, No. 4 .

NAGULESWARAN, S. and WILLIAMS, C.J.H.

'Lateral Vibration of a Pipe Conveying a Fluid'

J. Mech. Eng., 1968, Vol. 10, No. 3. 
APPENDIX A

SMALL RIGID-BODY MOTIONS

We wish to show that the strains resulting from rigid-body motions vanish for kluge's theory.

An arbitrary point in the space occupied by a thin shell is defined by the vector $\vec{R}\left(\alpha_{1}, \alpha_{2}, \rho\right)=\vec{r}\left(\alpha_{1}, \alpha_{2}\right)+\rho \vec{n}\left(\alpha_{1}, \alpha_{2}\right)$ where $\vec{r}$ is the position vector of a corresponding point on the reference surface, $\vec{n}$ is the unit normal vector from the reference surface and $\mathcal{J}$ denotes the distance of the point from the corresponding point on the reference surface along $\vec{\eta} \cdot \alpha_{1}$ and $\alpha_{2}$ are the parametric lines of the reference surface. We now define a displacement vector $\vec{U}\left(\alpha_{1}, \alpha_{2}, \rho\right)=U_{1}\left(\alpha_{1}, \alpha_{2}, \rho\right) \vec{t}_{1}+U_{2}\left(\alpha_{1}, \alpha_{2}, \rho\right) \vec{t}_{2}+W\left(\alpha_{1}, \alpha_{2}, \rho\right) \vec{n}$ where $\vec{t}_{1}, \vec{t}_{2}$ are unit vectors along the lines $\alpha_{1}$ and $\alpha_{2}$.

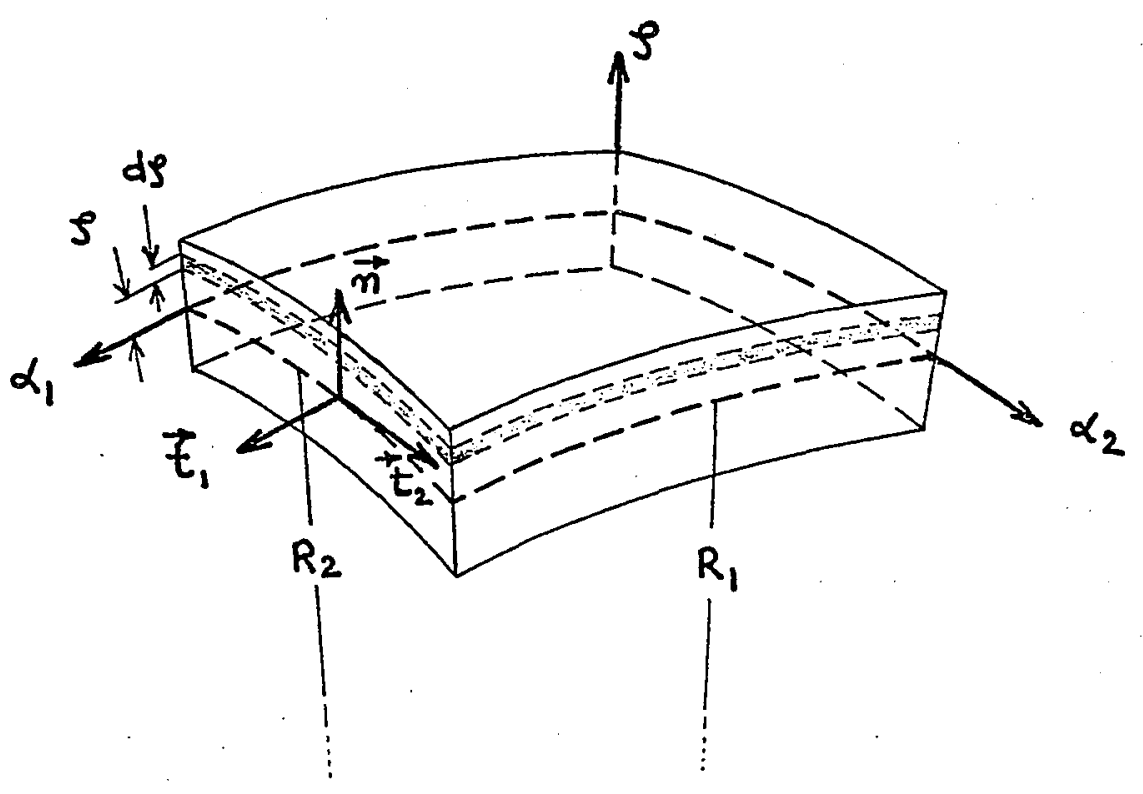


The assumption regarding the preservation of the normal element in a thin shell implies that the displacements are linearly distributed across the thickness of the shell:

$$
\begin{aligned}
& U_{1}\left(\alpha_{1}, \alpha_{2}, \rho\right)=u_{1}\left(\alpha_{1}, \alpha_{2}\right)+\rho \beta_{1}\left(\alpha_{1}, \alpha_{2}, \rho=0\right) \\
& U_{2}\left(\alpha_{1}, \alpha_{2}, \rho\right)=u_{2}\left(\alpha_{1}, \alpha_{2}\right)+\rho \beta_{2}\left(\alpha_{1}, \alpha_{2}, \rho=0\right) \\
& W\left(\alpha_{1}, \alpha_{2}, \rho\right)=\omega\left(\alpha_{1}, \alpha_{2}\right)
\end{aligned}
$$

The rotations $\beta_{1}$ and $\beta_{2}$ of tangents to the reference surface can be determined from the assumption that all strains in the direction of the normal to the reference surface vanish $\left(e_{n}=\nu_{1 n}=\nu_{2 n}=0\right)$ :

$$
\beta_{1}=\frac{u_{1}}{R_{1}}-\frac{1}{A_{1}} \frac{\partial \omega}{\partial \alpha_{1}} \quad ; \quad \beta_{2}=\frac{u_{2}}{R_{2}}-\frac{1}{A_{2}} \frac{\partial \omega}{\partial \alpha_{2}} \text {. }
$$

where $R_{1}, R_{2}$ are the principal radii of curvature and $A_{1}$, $\mathrm{A}_{2}$ are the coefficients in metric form of the reference surface. Using these notations and without neglecting $\rho / R_{i}$ with respect to unity as is done in the classical theory, the strains are given by the following equations:

$$
\begin{aligned}
& e_{n}=\gamma_{1 n}=\gamma_{2 n}=0 \\
& e_{1}=\frac{1}{1+\rho / R_{1}}\left(e_{1}^{0}+\rho k_{1}\right) ; \quad e_{2}=\frac{1}{1+\rho / R_{2}}\left(e_{2}^{0}+\rho k_{2}\right) \\
& \gamma_{12}=\frac{1}{\left(1+\rho / R_{1}\right)\left(1+\rho / R_{2}\right)}\left\{\gamma_{12}^{0}\left(1-\rho^{2} / R_{1} R_{2}\right)+2 \rho k_{12}^{0}\left[1+\frac{\rho}{2}\left(\frac{1}{R_{1}}+\frac{1}{R_{2}}\right)\right]\right\}
\end{aligned}
$$


- AB -

where:

$$
\begin{aligned}
& e_{1}^{0}=\frac{1}{A_{1}} \frac{\partial u_{1}}{\partial \alpha_{1}}+\frac{u_{2}}{A_{1} A_{2}} \frac{\partial A_{1}}{\partial \alpha_{2}}+\frac{w}{R_{1}} ; e_{2}^{0}=\frac{1}{A_{2}} \frac{\partial u_{2}}{\partial \alpha_{2}}+\frac{u_{1}}{A_{1} A_{2}} \frac{\partial A_{2}}{\partial \alpha_{1}}+\frac{w}{R_{2}} \\
& \gamma_{12}^{0}=\frac{A_{2}}{A_{1}} \frac{\partial}{\partial \alpha_{1}}\left(\frac{u_{2}}{A_{2}}\right)+\frac{A_{1}}{A_{2}} \frac{\partial}{\partial \alpha_{2}}\left(\frac{u_{1}}{A_{1}}\right)=\beta_{1}^{0}+\beta_{2}^{0} \\
& K_{1}=\frac{1}{A_{1}} \frac{\partial \beta_{1}}{\partial \alpha_{1}}+\frac{\beta_{2}}{A_{1} A_{2}} \frac{\partial A_{1}}{\partial \alpha_{2}} ; K_{2}=\frac{1}{A_{2}} \frac{\partial \beta_{2}}{\partial \alpha_{2}}+\frac{\beta_{1}}{A_{1} A_{2}} \frac{\partial A_{2}}{\partial \alpha_{1}} \\
& \tau=\frac{A_{2}}{A_{1}} \frac{\partial}{\partial \alpha_{1}}\left(\frac{\beta_{2}}{A_{2}}\right)+\frac{A_{1}}{A_{2}} \frac{\partial}{\partial \alpha_{2}}\left(\frac{\beta_{1}}{A_{1}}\right) \\
& 2 K_{12}^{0}=\tau+\frac{\beta_{2}^{0}}{R_{1}}+\frac{\beta_{1}^{0}}{R_{2}} \frac{u_{1}}{A_{1} A_{2}} \frac{\partial A_{1}}{\partial \alpha_{2}} ; \beta_{2}^{0}=\frac{1}{A_{2}} \frac{\partial u_{1}}{\partial \alpha_{2}}-\frac{u_{2}}{A_{1} A_{2}} \frac{\partial A_{2}}{\partial \alpha_{1}} \\
& B_{1}^{0}=\frac{1}{A_{1}} \frac{\partial u_{2}}{\partial \alpha_{1}}-\frac{u_{1}}{A_{1}}
\end{aligned}
$$

Let us now introduce small, constant, rigid-body motions; . they are defined by the vectors

$$
\begin{aligned}
& \vec{\Delta}=\delta_{1} \vec{t}_{1}+\delta_{2} \vec{t}_{2}+\delta_{n} \vec{n} \\
& \vec{\Omega}=-\omega_{2} \vec{t}_{1}+\omega_{1} \vec{t}_{2}+\omega_{n} \vec{n}
\end{aligned}
$$

For such motions, the displacement vector of points on the reference surface of the shell is given by

$$
\vec{u}=\vec{\Delta}+\vec{\Omega} \times \vec{r}
$$

where

$$
\vec{r}=p_{1} \vec{t}_{1}+p_{2} \vec{t}_{2}+\rho_{n} \vec{n}
$$

Using the equations $\frac{\partial \vec{\Delta}}{\partial \alpha_{1}}=\frac{\partial \vec{\Delta}}{\partial \alpha_{2}}=0$ and $\frac{\partial \vec{\Omega}}{\partial \alpha_{1}}=\frac{\partial \vec{\Omega}}{\partial \alpha_{2}}=0$ stating that the motions are constant, and the classical relations for the derivatives of $\vec{t}_{1}, \vec{t}_{2}, \vec{n}$ and $\vec{r}$ with respect to $\alpha_{1}, \alpha_{2}$, the following relations may be derived: 
$-\mathrm{A} 4$ -

$$
\begin{aligned}
& \begin{cases}\frac{\partial P_{1}}{\partial \alpha_{1}}=A_{1}-\frac{P_{2}}{A_{2}} \frac{\partial A_{1}}{\partial \alpha_{2}}-\frac{A_{1} P_{n}}{R_{1}} & ; \frac{\partial P_{1}}{\partial \alpha_{2}}=\frac{P_{2}}{A_{1}} \frac{\partial A_{2}}{\partial \alpha_{1}} \\
\frac{\partial P_{2}}{\partial \alpha_{1}}=\frac{P_{1}}{A_{2}} \frac{\partial A_{1}}{\partial \alpha_{2}} & ; \frac{\partial P_{2}}{\partial \alpha_{2}}=A_{2}-\frac{P_{1}}{A_{1}} \frac{\partial A_{2}}{\partial \alpha_{1}}-\frac{A_{2} P_{n}}{R_{2}} \\
\frac{\partial P_{n}}{\partial \alpha_{1}}=\frac{A_{1} P_{1}}{R_{1}} & ; \frac{\partial P_{n}}{\partial \alpha_{2}}=\frac{A_{2} P_{2}}{R_{2}}\end{cases} \\
& \begin{cases}\frac{\partial \delta_{1}}{\partial \alpha_{1}}=-\frac{\delta_{2}}{A_{2}} \frac{\partial A_{1}}{\partial \alpha_{2}}-\frac{A_{1} \delta_{n}}{R_{1}} & ; \frac{\partial \delta_{1}}{\partial \alpha_{2}}=\frac{\delta_{2}}{A_{1}} \frac{\partial A_{2}}{\partial \alpha_{1}} \\
\frac{\partial \delta_{2}}{\partial \alpha_{1}}=\frac{\delta_{1}}{A_{2}} \frac{\partial A_{1}}{\partial \alpha_{2}} & ; \frac{\partial \delta_{2}}{\partial \alpha_{2}}=-\frac{\delta_{1}}{A_{1}} \frac{\partial A_{2}}{\partial \alpha_{1}}-\frac{A_{2} \delta_{n}}{R_{2}} \\
\frac{\partial \delta_{n}}{\partial \alpha_{1}}=\frac{A_{1} \delta_{1}}{R_{1}} & ; \frac{\partial \delta_{n}}{\partial \alpha_{2}}=\frac{A_{2} \delta_{2}}{R_{2}}\end{cases} \\
& \begin{cases}\frac{\partial \omega_{1}}{\partial \alpha_{1}}=-\frac{\omega_{2}}{A_{2}} \frac{\partial A_{1}}{\partial \alpha_{2}} & ; \frac{\partial \omega_{1}}{\partial \alpha_{2}}=\frac{\omega_{2}}{A_{1}} \frac{\partial A_{2}}{\partial \alpha_{1}}-\frac{A_{2} \omega_{n}}{R_{2}} \\
\frac{\partial \omega_{2}}{\partial \alpha_{1}}=\frac{\omega_{1}}{A_{2}} \frac{\partial A_{1}}{\partial \alpha_{2}}+\frac{A_{1} \omega_{n}}{R_{1}} ; \frac{\partial \omega_{2}}{\partial \alpha_{2}}=-\frac{\omega_{1}}{A_{1}} \frac{\partial A_{2}}{\partial \alpha_{1}} \\
\frac{\partial \omega_{n}}{\partial \alpha_{1}}=-\frac{A_{1} \omega_{2}}{R_{1}} & ; \frac{\partial \omega_{n}}{\partial \alpha_{2}}=\frac{A_{2} \omega_{1}}{R_{2}}\end{cases}
\end{aligned}
$$

With the use of these relations it can be shown that the strains vanish when rigid-body displacements and rotations are imposed. For example, let us consider $\gamma_{12}^{0}$ and $K_{12}^{0}$.

$$
\begin{aligned}
\beta_{1}^{0}= & \frac{1}{A_{1}} \frac{\partial u_{2}}{\partial \alpha_{1}}-\frac{u_{1}}{A_{1} A_{2}} \frac{\partial A_{1}}{\partial \alpha_{2}}=\frac{1}{A_{1}} \frac{\partial}{\partial \alpha_{1}}\left(\delta_{2}+\rho_{n} \omega_{2}+\rho_{1} \omega_{n}\right)-\frac{\delta_{1}+P_{n} \omega_{1}-\rho_{2} \omega_{n}}{A_{1} A_{2}} \frac{\partial A}{\partial \alpha} \\
= & \frac{1}{A_{1}}\left[\frac{\delta_{1}}{A_{2}} \frac{\partial A_{1}}{\partial \alpha_{2}}+\rho_{n}\left(\frac{\omega_{1}}{A_{2}} \frac{\partial A_{1}}{\partial \alpha_{2}}+\frac{A_{1} \omega_{n}}{R_{1}}\right)+\omega_{2}\left(\frac{A_{1} P_{1}}{R_{1}}\right)+\rho_{1}\left(-\frac{A_{1} \omega_{2}}{R_{1}}\right)\right. \\
& \left.+\omega_{n}\left(A_{1}-\frac{\rho_{2}}{A_{2}} \frac{\partial A_{1}}{\partial \alpha_{2}}-\frac{A_{1} \rho_{n}}{R_{1}}\right)\right]-\frac{\delta_{1}+\rho_{n} \omega_{1}-\rho_{2} \omega_{n}}{A_{1} A_{2}} \frac{\partial A_{1}}{\partial \alpha_{2}} \\
\beta_{1}^{0}= & \omega_{n}
\end{aligned}
$$


- AS -

To obtain $\beta_{2}^{0}$, we change the suffices 1 to 2,2 to 1 and $\omega_{\mathrm{n}}$ to ${ }^{-\omega_{\mathrm{n}}}$ so that:

$$
\beta_{2}^{0}=-\omega_{n}
$$

We therefore have $\quad \boldsymbol{\gamma}_{12}^{0}=\beta_{1}^{0}+\beta_{2}^{0}=\omega_{n}-\omega_{n}=0$

$$
\tau=\frac{1}{A_{1}} \frac{\partial \beta_{2}}{\partial \alpha_{1}}-\frac{\beta_{2}}{A_{1} A_{2}} \frac{\partial A_{2}}{\partial \alpha_{1}}+\frac{1}{A_{2}} \frac{\partial \beta_{1}}{\partial \alpha_{2}}-\frac{\beta_{1}}{A_{1} A_{2}} \frac{\partial A_{1}}{\partial \alpha_{2}}
$$

We replace the rotations $\beta_{1}, \beta_{2}$ by $\omega_{1}$ and $\omega_{2}$

$$
\begin{aligned}
& \tau=\frac{1}{A_{1}}\left(\frac{\omega_{1}}{A_{2}} \frac{\partial A_{1}}{\partial \alpha_{2}}+\frac{A_{1} \omega_{n}}{R_{1}}\right)-\frac{\omega_{2}}{A_{1} A_{2}} \frac{\partial A_{2}}{\partial \alpha_{1}}+\frac{1}{A_{2}}\left(\frac{\omega_{2}}{A_{1}} \frac{\partial A_{2}}{\partial \alpha_{1}}-\frac{A_{2} \omega_{n}}{R_{2}}\right)-\frac{\omega_{1}}{A_{1} A_{2}} \frac{\partial A_{1}}{\partial \alpha_{2}} \\
& \tau=\left(\frac{1}{R_{1}}-\frac{1}{R_{2}}\right) \omega_{n}
\end{aligned}
$$

We therefore have

$$
\begin{aligned}
2 K_{12}^{0} & =\tau+\frac{\beta_{2}^{0}}{R_{1}}+\frac{\beta_{1}^{0}}{R_{2}}=\left(\frac{1}{R_{1}}-\frac{1}{R_{2}}\right) \omega_{n}-\frac{\omega_{n}}{R_{1}}+\frac{\omega_{n}}{R_{2}}=0 \\
K_{12}^{0} & =0
\end{aligned}
$$

Similarly we can show that $K_{1}=K_{2}=0$ and $e_{1}^{0}=e_{2}^{0}=0$ The evanescence of the coefficients $e_{1}^{0}, e_{2}^{0}, K_{1}, K_{2}, \gamma_{12}^{0}$ and $K_{12}^{0}$ shows that the strains vanish for small rigid-body motions. 


\section{APPENDIX B \\ NATURAL FREQUENCIES OF THIN CIRCULAR \\ CYIINDRICAL SHELIS IN VACUO}

The boundary value problem consists of an eighth-order homogeneous system of three partial differential equations

$u^{\prime \prime}+\frac{1-\nu}{2} u^{\prime \prime}+\frac{1+\nu}{2} v^{\prime \prime}+\nu w^{\prime}+k\left[\frac{1-\nu}{2} u^{\prime \prime}-w^{\prime \prime \prime}+\frac{1-\nu}{2} w^{\prime} \cdot 0\right]=0$ $\frac{1+2}{2} u^{\prime \prime}+v^{\prime \prime}+\frac{1-2}{2} v^{\prime \prime}+w^{\prime}+k\left[\frac{3}{2}(1-2) v^{\prime \prime}-\frac{3-2}{2} w^{\prime \prime} \cdot\right]=0$ $v u^{\prime}+v^{\prime}+w+k\left[\frac{1-2)}{2} u^{\prime \cdots}-u^{\prime \prime \prime}-\frac{3-\nu}{2} v^{\prime \prime}+\nabla^{4} w+2 w^{\prime}+w\right]=0$ and associated boundary conditions. [The symbols $(-)$ ' and $(-)$ are used for a $\frac{\partial(-)}{\partial x}$ and $\frac{\partial(-)}{\partial \theta}$ respectively; $\left.k=h^{2} / 12 a^{2} \cdot\right]$

On a clamped edge, these boundary conditions are $u=v=w=0$, $\partial w / \partial x=0$, whereas on a free edge, the four essential forces and moments are zero: $\mathrm{N}_{\mathrm{X}}=\mathrm{S}_{\mathrm{X}}=\mathrm{T}_{\mathrm{X}}=0, \mathrm{M}_{\mathrm{X}}=0$. Introducing the displacements, these latter conditions give:
$N_{x}=0 \Rightarrow$
$u^{\prime}+2 v^{\circ}+2 w-k w^{\prime \prime}$
$=0$
$M_{x}=0 \Rightarrow$
$w^{\prime \prime}+\nu w^{-*}-2 v-u^{\prime}$
$=0$
$S_{x}=0 \Rightarrow$
$w^{\prime \prime}+(2-v) w^{\prime \prime}-\frac{3-v}{2} v^{\prime \prime}+$
$u^{\prime}+v^{\prime}+k\left(3 v^{\prime}-3 w^{\prime \cdot}\right)$
$=0$
$T_{x}=0 \Rightarrow$
$=0$ 
$-\mathrm{B} 2-$

A general solution of the form

$$
\begin{aligned}
& u=\sum_{j=1}^{8} A_{j} \exp \left[i\left(\lambda_{j} \frac{x}{a}+n \theta+\omega t\right)\right] \\
& v=\sum_{j=1}^{8} B_{j} \exp \left[i\left(\lambda_{j} \frac{x}{a}+n \theta+\omega t\right)\right] \\
& \omega=\sum_{j=1}^{8} C_{j} \exp \left[i\left(\lambda_{j} \frac{x}{a}+n \theta+\omega t\right)\right]
\end{aligned}
$$

is assumed.

Substitution of these expressions in the

differential system leads to three linear, homogeneous equations in the unknowns $A_{j}, B_{j}$ and $C_{j}$.

$$
\begin{array}{ll}
A_{j}\left[\lambda^{2}+\frac{1-\nu}{2} n^{2}(1+k)-\Omega^{2}\right]+B_{j}\left[\frac{1+\nu}{2} \lambda n\right]-i \lambda C_{j}\left[\nu+k\left(\lambda^{2}-\frac{1-\nu}{2} n^{2}\right)\right] & =0 \\
A_{j}\left[\frac{1+\nu}{2} \lambda n\right]+B_{j}\left[n^{2}+\frac{1-\nu}{2} \lambda^{2}(1+3 k)-\Omega^{2}\right]-i C_{j}\left[n+\frac{3-\nu}{2} \lambda^{2} n k\right] & =0 \\
A_{j} \lambda\left[\nu+k\left(\lambda^{2}-\frac{1-\nu}{2} n^{2}\right)\right]+B_{j}\left[n+\frac{3-\nu}{2} \lambda^{2} n k\right]-i C_{j}\left[1+k\left\{\left(\lambda^{2}+n^{2}\right)^{2}-2 n^{2}+1\right\}-\Omega^{2}\right]=0
\end{array}
$$

where $\Omega^{2}=\gamma \omega^{2}=a^{2} P_{s} \frac{1-\nu^{2}}{E} \omega^{2}$.

For nontrivial solutions, the associated determinant

$$
\begin{aligned}
& \text { must be zero, } \\
& \lambda^{2}+\frac{1-2}{2} n^{2}(1+k)-\Omega^{2} \quad \frac{1+\nu}{2} \lambda n \quad \lambda\left[v+k\left(\lambda^{2}-\frac{1-\nu}{2} n^{2}\right)\right] \\
& \frac{1+\nu}{2} \lambda_{n} \quad n^{2}+\frac{1-v}{2} \lambda^{2}(1+3 k)-\Omega^{2} \quad n+\frac{3-2}{2} \lambda^{2} n k \\
& \lambda\left[\nu+k\left(\lambda^{2}-\frac{1-\nu}{2} n^{2}\right)\right] \quad n+\frac{3-\nu}{2} \lambda^{2} n k \quad 1+k\left[\left(\lambda^{2}+n^{2}\right)^{2}-2 n^{2}+1\right]-\Omega^{2} \\
& A_{j}=\alpha_{j} C_{j} ; B_{j}=\beta_{j} C_{j}
\end{aligned}
$$

where

$$
\alpha_{j}=(-i) \frac{\frac{1+\nu}{2} \lambda n\left(n+\frac{3-\nu}{2} \lambda^{2} n k\right)-\lambda\left[n^{2}+\frac{1-\nu}{2} \lambda^{2}(1+3 k)-\Omega^{2}\right]\left[2+k\left(\lambda^{2}-\frac{1-\nu}{2} n^{2}\right)\right]}{\left[\lambda^{2}+\frac{1-\nu}{2} n^{2}(1+k)-\Omega^{2}\right]\left[n^{2}+\frac{1-\nu}{2}(1+3 k)-\Omega^{2}\right]-\left(\frac{1+\nu}{2} \lambda\right)^{2}}
$$


and $\beta_{j}=(-i) \frac{\left[\nu+k\left(\lambda^{2}-\frac{1-\nu}{2} n^{2}\right)\right] \frac{1+\nu}{2} \lambda^{2} n-\left[\lambda^{2}+\frac{1-\nu}{2} n^{2}(1+k)-\Omega^{2}\right]\left(n+\frac{3-\nu}{2} \lambda^{2} n k\right)}{\left[\lambda^{2}+\frac{1-\nu}{2} n^{2}(1+k)-\Omega^{2}\right]\left[n^{2}+\frac{1-\nu}{2}(1+3 k)-\Omega^{2}\right]-\left(\frac{1+2}{2} \lambda n\right)^{2}}$.

The boundary conditions must now be satisfied. Let us take, for example, the case of a shell clamped at both ends; this means

$$
u=v=w=0 ; \quad \partial w / \partial x=0 \text {, at both } x=0 \text { and } x=\ell
$$

These conditions lead to a system of eight linear homogeneous equations in the unknowns $c_{j}(j=1,2, \ldots, 8)$
At $x=0$

$$
\left\{\begin{array}{c}
u=0 \\
v=0 \\
w=0 \\
\partial w / \partial x=0
\end{array}\right.
$$$$
\sum_{j=1}^{8} \alpha_{j} c_{j}
$$$$
=0
$$$$
\sum \beta_{j} C_{j}
$$$$
\Sigma C_{j}
$$
At $x=l \quad\left\{\begin{aligned} u & =0 \\ v & =0 \\ w & =0 \\ \partial w / \partial x & =0\end{aligned}\right.$$$
\sum \lambda_{j} C_{j}
$$$$
=0
$$$$
=0
$$$$
=0
$$$$
\sum \alpha_{i} C_{j} \exp \left(i \lambda_{j} l / a\right)
$$$$
=0
$$$$
\sum \beta_{j} C_{j} \exp \left(i \lambda_{j} l / a\right)
$$$$
=0
$$$$
\sum C_{j} \exp \left(i \lambda_{j} l / a\right)
$$$$
=0
$$$$
\sum \lambda_{j} C_{j} \exp \left(i \lambda_{j} l / a\right)
$$$$
=0
$$

Again for non-trivial solutions the associated determinant must be zero,

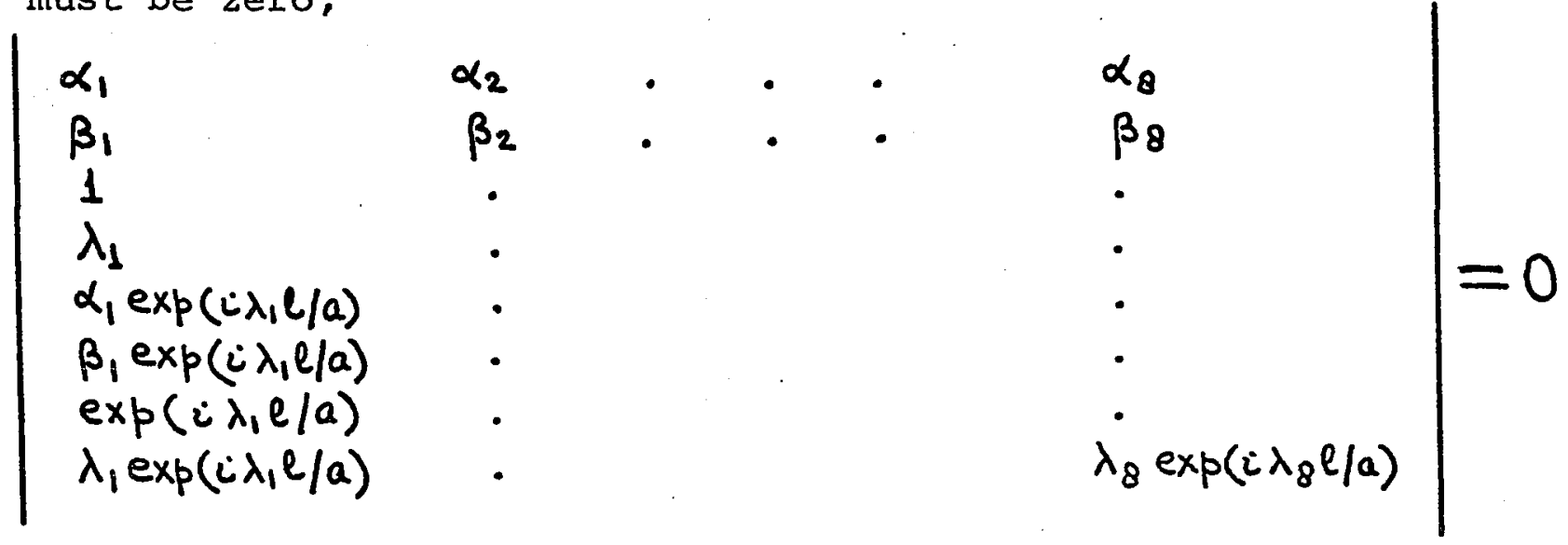


this is the frequency equation.

If the frequency $\omega$ is a natural frequency, the frequency equation will be satisfied. The solution of. the equations with unknowns $c_{j}$ will allow the complete determination of the functions $u(x, \theta, t), v(x, \theta, t)$, $w(x, \theta, t)$ and, hence, the determination of the modal shapes.

Summarizing, the determination of the natural frequencies of thin circular cylindrical shells involves the simultaneous solution of the characteristic and frequency equations, writțen in determinantal form. There are several ways to tackle the numerical aspect of the problem. We chose a direct trial and error approach; this method requires the use of a digital computer. Copies of programs are given in Appendix E. 


\section{APPENDIX C \\ VIBRATIONS OF THE PIPE WITH FLOW}

\section{C.1 Pressurization and Curvature Effects}

In the derivation of the equations the change of curvature of the element was not considered; if we take it into consideration, the equations of equilibrium may be written

$a \frac{\partial N_{x}}{\partial x}+\frac{\partial N_{\theta x}}{\partial \theta}+a Q_{x} \frac{\partial^{2} \omega}{\partial x^{2}}-a N_{x \theta} \frac{\partial^{2} v}{\partial x^{2}}-Q_{\theta}\left(\frac{\partial v}{\partial x}-\frac{\partial^{2} w}{\partial x^{\partial \theta}}\right)-N_{\theta}\left(\frac{\partial^{2} v}{\partial x \partial \theta}+\frac{\partial w}{\partial x}\right)+a q_{x}=0$

$a \frac{\partial N_{x \theta}}{\partial x}+\frac{\partial N_{\theta}}{\partial \theta}-Q_{\theta}+a N_{x} \frac{\partial^{2} v}{\partial x^{2}}-Q_{x}\left(\frac{\partial v}{\partial x}-\frac{\partial^{2} w}{\partial x \partial \theta}\right)+N_{\theta x}\left(\frac{\partial^{2} v}{\partial x \partial \theta}+\frac{\partial w}{\partial x}\right)-Q_{\theta}\left(\frac{1}{a} \frac{\partial v}{\partial \theta}-\frac{1}{a} \frac{\partial^{2} w}{\partial \theta^{2}}\right)=0$

$a \frac{\partial Q_{x}}{\partial x}+\frac{\partial Q_{\theta}}{\partial \theta}+N_{\theta}+N_{x \theta}\left(\frac{\partial v}{\partial x}-\frac{\partial^{2} w}{\partial x \partial \theta}\right)-a N_{x} \frac{\partial^{2} w}{\partial x^{2}}+N_{\theta}\left(\frac{1}{a} \frac{\partial v}{\partial \theta}-\frac{1}{a} \frac{\partial^{2} w}{\partial \theta^{2}}\right)+N_{\theta x}\left(\frac{\partial v}{\partial x}-\frac{\partial^{2} w}{\partial x \partial \theta}\right)-a p=0$

where the normal and transverse shearing forces $Q_{x}$ and $Q_{\theta}$ are governed by the equations:

$$
\begin{aligned}
& a \frac{\partial M_{x}}{\partial x}+\frac{\partial M_{\theta x}}{\partial \theta}-a Q_{x}+a M_{x \theta} \frac{\partial^{2} v}{\partial x^{2}}-M_{\theta}\left(\frac{\partial^{2} v}{\partial x \partial \theta}+\frac{\partial w}{\partial x}\right)=0 \\
& a \frac{\partial M_{x \theta}}{\partial x}-\frac{\partial M_{\theta}}{\partial \theta}+a Q_{\theta}-a M_{x} \frac{\partial^{2} v}{\partial x^{2}}-M_{\theta x}\left(\frac{\partial^{2} v}{\partial x \partial \theta}+\frac{\partial w}{\partial x}\right)=0
\end{aligned}
$$

Note - The notation here is that used by Timoshenko ${ }^{[8]}$; in particular, $w$ is changed to $-w$.

$$
\text { If the forces and moments } \mathrm{N}_{\mathbf{X}}, \mathrm{N}_{\theta} \text {, are small }
$$

in comparison with their critical values at which lateral buckling of the shell may occur, their effect on bending is negligible, and we can omit all terms containing products 
of the resultant forces or resultant moments with derivatives of the small displacements $u, v$ and $w$.

In our case, the shell is very thin and collapses in the absence of internal pressure; therefore, it is prudent to examine the influence of these particular terms.

We assume that under the action of uniform internal pressure the circular cylindrical shell remains circular and we consider'small deflections from this uniformly compressed form of equilibrium. The stress resultants and couples are composed of steady components due to pressurization and components due to deformation; but all. resultant forces except $\mathrm{N}_{\mathrm{X}}$ and $\mathrm{N}_{\theta}$ and all resultant couples are very small, and we can neglect the products of these with derivatives of the displacements $u, v$ and $w$, obtaining

$$
\begin{aligned}
& u=u_{1} ; v=v_{1} ; w=w_{0}+w_{1} \text { since } u_{0}=v_{0}=0 \\
& p=p_{0}+p_{1} \\
& N_{x}=N_{x_{0}}+N_{x_{1}} ; N_{\theta}=N_{\theta 0}+N_{\theta 1} \\
& N_{x \theta}=N_{x \theta 1} \quad ; \quad M_{x}=M_{x 1} \\
& \text { - } \\
& \text { Since } N_{x \theta 0}=0 ; M_{x 0}=0 ; \cdots
\end{aligned}
$$

A further refinement may be introduced by taking into consideration the stretching of the reference surface which amounts to replacing $N_{\theta}$ by $N_{\theta}\left(1+e_{x}\right)$ and $p$ by $p\left(1+e_{x}\right)\left(1+e_{\theta}\right)$, where the strains $e_{x}$ and $e_{\theta}$ are given by the relations 


$$
e_{x}=\frac{\partial u}{\partial x} ; e_{\theta}=\frac{1}{a} \frac{\partial v}{\partial \theta}+\frac{w}{a}
$$

Thus, we introduce the following supplementary terms in the equations:

1. First Equation: $\quad-\frac{1-v^{2}}{E h}\left(N_{\theta 0}+N_{\theta 1}\right)\left(\frac{\partial^{2} v_{1}}{\partial x \partial \theta}+\frac{\partial w_{0}}{\partial x}+\frac{\partial w_{1}}{\partial x}\right)$

2. Second Equation: $\frac{1-\nu^{2}}{E h}\left(N_{x_{0}}+N_{x 1}\right) \frac{\partial^{2} v_{1}}{\partial x^{2}}$

3. Third Equation: $\quad \frac{1-v^{2}}{E h}\left(N_{\theta 0}+N_{\theta_{1}}\right)\left(1+\frac{1}{a} \frac{\partial^{2} v_{1}}{\partial x^{2}}-\frac{1}{a} \frac{\partial^{2} w_{1}}{\partial \theta^{2}}\right)\left(1+\frac{\partial u_{1}}{\partial x}\right)$

$$
-a \frac{1-v^{2}}{E h}\left(N_{x 0}+N_{x 1}\right)\left(\frac{\partial^{2} \omega_{0}}{\partial x^{2}}+\frac{\partial^{2} w_{1}}{\partial x^{2}}\right)
$$

Practically, the shell remains cylindrical under steady pressurization, so that the term $\partial \omega_{0} / \partial x$ is equal to zero. The zeroth-order equations are then

$$
\begin{array}{ll}
\frac{\partial N_{x_{0}}}{\partial x}+9 x & =0 \\
\frac{\partial N_{\theta_{0}}}{\partial \theta} & =0 \\
N_{\theta 0}-a p & =0
\end{array}
$$

and the first order equations of motion are

$$
\begin{aligned}
u_{1}^{\prime \prime}+\frac{1-\nu}{2} u_{i}+\ldots+k\left[\frac{1-v}{2} u_{i}-w_{1}^{\prime \prime}+\frac{1-v}{2} w_{1}^{\prime \cdot}\right]-\sigma_{\theta}\left(v_{1}^{\prime}+w_{1}^{\prime}\right) & =\gamma \frac{\partial^{2} u_{1}}{\partial t^{2}} \\
\left.\frac{1+v}{2} u_{1}^{\prime}+\cdots+k+\frac{3}{2}(1-v) v_{1}^{\prime \prime}-\frac{3-\nu}{2} w_{1}^{\prime \prime}\right]+\sigma_{x} v_{1}^{\prime \prime} & =\gamma \frac{\partial^{2} v_{1}}{\partial t^{2}} \\
v u_{1}^{\prime}+v_{i}^{\prime}+w_{1}+k\left[\cdots+\nabla^{4} w_{1}+2 w_{i}^{\prime}+w_{1}\right]-\sigma_{\theta}\left(w_{i}^{\prime \prime}+w_{1}\right) & \\
-\sigma_{x} w_{1}^{\prime \prime}-a \mathbb{P} & =-\gamma \frac{\partial^{2} w_{1}}{\partial t^{2}}
\end{aligned}
$$


$-\mathrm{C} 4-$

Where $\quad \sigma_{\theta}=\frac{1-\nu^{2}}{E h} N_{\theta 0} ; \sigma_{x}=\frac{1-\nu^{2}}{E h} N_{x_{0}}$

$$
\mathbb{P}=\frac{1-v^{2}}{E h} a p_{1} ; \gamma=a^{2} P_{s} \frac{1-\nu^{2}}{E h}
$$

The normal and transverse forces $N_{x o}$ and Neo are defined by the steady-state equations 'which, upon integration, give:

$$
\begin{aligned}
& N_{x 0}=q_{x}(l-x) \\
& N_{\theta 0}=a p_{0}(x)
\end{aligned}
$$

The pressure distribution $p_{0}(x)$ is found by considering the equilibrium of a small cylinder of fluid, of length $d x$ and radius $a, i . e$.

$$
\begin{gathered}
\left(p_{0}+\frac{\partial p_{0}}{\partial x} d x\right) \pi a^{2}-p_{0} \pi a^{2}+q_{x} 2 \pi a d x=0 \\
\frac{\partial p_{0}}{\partial x}+\frac{2 q_{x}}{a}=0
\end{gathered}
$$

Since the outlet pressure is zero, the integration leads to

$$
p_{0}(x)=\frac{29 x}{a}(l-x)
$$

Eventually $\bar{\zeta}_{x}$ and $\bar{\zeta}_{\theta}$ are defined as

$$
\begin{aligned}
& \sigma_{x}=\frac{1-y^{2}}{E h} q_{x}(l-x) \\
& \sigma_{\theta}=2 \frac{1-y^{2}}{E h} q_{x}(l-x)=2 \sigma_{x}
\end{aligned}
$$

where $q_{x}$ is determined experimentally. 
$-\mathrm{C5}-$

C. 2 Equations of Fluid Flow

In cylindrical polar coordinates, the equation defining the flow perturbation potential $\phi$ is

$$
\frac{1}{r} \frac{\partial}{\partial r}\left(r \frac{\partial \phi}{\partial r}\right)+\frac{1}{r^{2}} \frac{\partial^{2} \phi}{\partial \theta^{2}}+\frac{\partial^{2} \phi}{\partial x^{2}}=0
$$

The boundary conditions on the wall are

$$
\left.\begin{array}{l}
V_{r}=\frac{\partial \omega}{\partial t}+U \frac{\partial w}{\partial x}=\frac{\partial \phi}{\partial r} \\
V_{\theta}=\frac{1}{r} \frac{\partial \phi}{\partial \theta} \\
V_{x}=U+\frac{\partial \phi}{\partial x}
\end{array}\right\} \text { at } r=a
$$

We recall here that the boundary conditions are taken at $r=a$, which means that we assume the displacements of the wall to be small and the shell to be thin. The pressure on the wall is given by Bernoulli's equation

$$
\frac{\partial \phi}{\partial t}+\frac{v^{2}}{2}+\left.\frac{p}{p}\right|_{r=a}=0
$$

The square of the velocity may be written as follows:

$$
\begin{aligned}
& V^{2}=\left(\frac{\partial \phi}{\partial x}+U\right)^{2}+\left(\frac{1}{r} \frac{\partial \phi}{\partial \theta}\right)^{2}+\left(\frac{\partial \phi}{\partial r}\right)^{2} \\
& \text { or } V^{2}=U^{2} \text { (zeroth order term) }+2 U \frac{\partial \phi}{\partial x} \text { (first-order term) } \\
& +\left[\left(\frac{\partial \phi}{\partial x}\right)^{2}+\left(\frac{1}{r} \frac{\partial \phi}{\partial \theta}\right)^{2}+\left(\frac{\partial \phi}{\partial r}\right)^{2}\right] \text { (second-order terms) }
\end{aligned}
$$


$-\mathrm{c6}-$

Retaining only first-order terms we obtain

$$
\frac{\partial \phi}{\partial t}+U \frac{\partial \phi}{\partial x}+\left.\frac{p_{1}}{P}\right|_{r=a}=0
$$

or

$$
P_{1}=-P\left[\frac{\partial \phi}{\partial t}+U \frac{\partial \phi}{\partial x}\right]_{r=a}
$$

We assume a separable solution of the form

$$
\phi(x, \theta, r, t)=R(r) S(x, \theta, t)
$$

The derivative of $\phi$ with respect to $\Omega$ taken at the wall, . is then

$$
\left.\frac{\partial \phi}{\partial r}\right|_{r=a}=R^{\prime}(a) \cdot S(x, \theta, t)=\left[\frac{\partial w}{\partial t}+U \frac{\partial w}{\partial x}\right]_{r=a}
$$

where the boundary conditions on the wall were utilized. The function $\phi(x, \theta, r, t)$ may then be expressed as

$$
\phi(x, \theta, r, t)=\frac{R(r)}{R^{\prime}(a)}\left[\frac{\partial \omega}{\partial t}+U \frac{\partial \omega}{\partial x}\right]_{r=a}
$$

and similarly the pressure $p_{1}$ on the wall is

$$
P_{1}(x, \theta, t)=-\left.\rho \frac{R(a)}{R^{\prime}(a)}\left[\frac{\partial}{\partial t}+U \frac{\partial}{\partial x}\right]^{2} \omega\right|_{r=a}
$$

To obtain a solution of Laplace's equation $\nabla^{2} \phi=0$, we 
$-C 7-$

assume the function $s(x, \theta, t)$ to be of the form

$$
S(x, \theta, t)=S_{0} \exp \left[i\left(\lambda \frac{x}{a}+n \theta+\omega t\right)\right]
$$

This assumption seems logical since we have already assumed a similar form for the displacement $w$. Upon substitution into Laplace's equation, we obtain

$$
\begin{array}{ll}
\frac{1}{r} \frac{\partial}{\partial r}\left(r R^{\prime}\right)-\frac{n^{2}}{r^{2}} R S-\frac{\lambda^{2}}{a^{2}} R S=0 \\
R^{\prime \prime}+\frac{R^{\prime}}{r}-\left(\frac{n^{2}}{r^{2}}+\frac{\lambda^{2}}{a^{2}}\right) R=0
\end{array}
$$

Using the change of variables $y=\frac{\lambda}{a} r$, this equation becomes

$$
\frac{\partial^{2} R}{\partial y^{2}}+\frac{1}{y} \frac{\partial R}{\partial y}-\left(1-\frac{n^{2}}{y^{2}}\right) R=0 ;
$$

its solution may be expressed in terms of Bessel functions of the first and second kind, of order $n$,

$$
R(r)=B_{1}^{\prime} J_{n}\left(\frac{i \lambda}{a} r\right)+B_{2}^{\prime} Y_{n}\left(\frac{i \lambda}{a} r\right)
$$

or in terms of the modified Bessel functions of the first and second kind, of order $n$ (cf. Watson (18),

$$
R(r)=B_{1} I_{n}\left(\frac{\lambda}{a} r\right)+B_{2} K_{n}\left(\frac{\lambda}{a} r\right)
$$

This solution must be finite on the axis of the cylinder $(r=0)$; tills means we have to set $B_{2}$ equal to zero, and 
hence

$$
R(r)=B_{1} \operatorname{In}\left(\frac{\lambda}{a} r\right)
$$

Now if we want to take into account the inertia of the fluid (of density Pext) outside the shell, the previous derivation is perfectly valid. We simply have to set $U$ equal to zero, as this fluid is at rest under steady conditions of the shell, and to impose that the solution be finite at infinity $(r \rightarrow \infty)$, which means we have to take $B_{1}$ equal to zero. In this case the solution is then

$$
R(r)=B_{2} K_{n}\left(\frac{\lambda}{a} r\right)
$$

Eventually, the pressure acting on the shell walls is defined as

$$
P_{1}=-\rho \frac{I_{n}\left(\frac{\lambda}{a} r\right)}{\frac{\partial}{\partial r} I_{n}\left(\frac{\lambda}{a} r\right)}\left[\frac{\partial}{\partial t}+U \frac{\partial}{\partial x}\right]^{2} \omega+\left.P_{\text {ext. }} \frac{K_{n}\left(\frac{\lambda}{a} r\right)}{\frac{\partial}{\partial r} K_{n}\left(\frac{\lambda}{a} r\right)} \frac{\partial^{2} w}{\partial t^{2}}\right|_{r=a}
$$

Some further manipulation allows us to transform this expression to

$$
p_{1}=-\rho \frac{a}{n+\lambda \frac{I_{n+1}(\lambda)}{I_{n}(\lambda)}}\left[\frac{\partial}{\partial t}+U \frac{\partial}{\partial x}\right]^{2} w+\rho_{\text {ext. }} \frac{a}{n-\lambda \frac{K_{n+1}(\lambda)}{K_{n}(\lambda)}} \frac{\partial^{2} w}{\partial t^{2}}
$$

this last form will be used in the differential equations of motion.

At this stage, it is of interest to study the 
case of long waves corresponding to small values of $\lambda$ with respect to unity, and the limiting case of $\lambda$ equal to zero. The functions $\operatorname{In}(\lambda)$ and $K_{n}(\lambda)$ are defined by the following series:

$$
\begin{aligned}
I_{n}(\lambda)= & (i)^{-n} J_{n}\left(\lambda_{i}\right) ; J_{n}(\lambda)= \\
K_{n}(\lambda)= & \frac{1}{2} \sum_{m=0}^{n-1} \frac{(-1)^{m}(n-m) \frac{(\lambda / 2)^{n+2 r}}{r !(n+r) !}}{(\lambda ! r)^{n-2 m}}+(-1)^{n+1} \sum_{m=0}^{\infty} \frac{(\lambda / 2)^{n+2 m}}{m !(n+m) !}\left[\ln \left(\frac{\lambda}{2}\right)\right. \\
& \left.-\frac{1}{2} \psi(n+m+1)-\frac{1}{2} \psi(m+1)\right]
\end{aligned}
$$

where

$$
\begin{aligned}
& \Psi(1)=-\gamma \\
& \Psi(m+1)=\frac{1}{1}+\frac{1}{2}+\cdots+\frac{1}{m}-\gamma
\end{aligned}
$$

straight forward manipulation leads to the following relations

$$
\begin{aligned}
& \frac{I_{n+1}(\lambda)}{I_{n}(\lambda)}=\frac{\lambda}{2(n+1)}-\frac{\lambda^{3}}{8(n+1)^{2}(n+2)}+O\left(\lambda^{5}\right) \\
& \frac{K_{n+1}(\lambda)}{K_{n}(\lambda)} \simeq \frac{2 n}{\lambda}
\end{aligned}
$$

In the limiting case of infinite wavelength $(\lambda \rightarrow 0)$, the pressure term is then

$$
p_{1}=-p \frac{a}{n}\left[\frac{\partial}{\partial t}+U \frac{\partial}{\partial x}\right]^{2} w-P_{\text {ext. }} \frac{a}{n} \frac{\partial^{2} w}{\partial t^{2}}
$$

and for a circumferential wave number, $n$, equal to unity, corresponding to lateral (i.e. beam-type) oscillations of 
$-\mathrm{C} 10-$

the shell, the pressure term is

$$
p_{1}=-\rho a\left[\frac{\partial}{\partial t}+U \frac{\partial}{\partial x}\right]^{2} w-\rho_{\text {ext }} a \frac{\partial^{2} w}{\partial t^{2}}
$$




\section{APPENDIX D}

\section{EIGENFUNCTIONS IN THE PRESENCE OF FLOW}

The general solution in complex form

$$
w(x, \theta, t)=W(x) e^{i \Omega t} e^{i n \theta}
$$

may be written as

$w(x, \theta, t)=\left[W_{R}(x)+i W_{I}(x)\right][\cos (\Omega t+n \theta+\Psi)+i \sin (\Omega t+n \theta+\Psi)]$

The complete solution. is the sum of six terms, such as the one above. These terms correspond to the six frequencies $\Omega_{11}, \Omega_{12}, \Omega_{21}, \Omega_{22}, \Omega_{31}$, and $\Omega_{32}$, solutions of the characteristic equation. These frequencies are symmetrical, in pairs, with respect to the frequency imaginary axis, which means that if $\Omega_{j 1}, j=1,2,3$ is of the form $\Omega_{x}+i \Omega_{y}$, then $\Omega_{j 2}, j=1,2,3$ is of the form $-\Omega_{x}+i \Omega_{y}$. Actually, we are only interested in the first pair $\left(\Omega_{11}, \Omega_{12}\right)$. Once we have the solution corresponding to $\Omega_{11}$, it is easy to obtain the solution corresponding to $\Omega_{12}$. Changing $\Omega_{I 1}$ to $\Omega_{12}$ amounts to change

$$
\begin{aligned}
& \Omega=\Omega_{\mathrm{x}}+i \Omega_{\mathrm{y}} \quad \text { to }-\bar{\Omega}=-\Omega_{\mathrm{x}}+i \Omega_{\mathrm{y}} \text {, } \\
& \lambda=\lambda_{x}+i \lambda_{y} \text { to }-\bar{\lambda}=-\lambda_{x}+i \lambda_{y} \text {, } \\
& W=W_{R}(x)+i W_{I}(x) \text { to } \bar{W}=W_{R}-i W_{I} \text {, } \\
& \mathrm{U}=\mathrm{U}_{\mathrm{R}}(\mathrm{x})+\mathrm{i} \mathrm{U}_{\mathrm{I}}(\mathrm{x}) \text { to } \overline{\mathrm{U}}=\mathrm{U}_{\mathrm{R}}-i \mathrm{U}_{\mathrm{I}^{\prime}} \text {, } \\
& \mathrm{V}=\mathrm{V}_{\mathrm{R}}(\mathrm{x})+i \mathrm{~V}_{\mathrm{I}}(\mathrm{x}) \text { to }-\overline{\mathrm{V}}=-\mathrm{V}_{\mathrm{R}}+\mathrm{V}_{\mathrm{I}} \text {. }
\end{aligned}
$$

The solution is then in real form (real part of the complex solution) 


$$
\begin{aligned}
w(x, \theta, t)= & \cdot w_{R} \cos \left(\Omega t+n \theta+\Psi_{I}\right)-W_{I} \sin \left(\Omega t+n \theta+\Psi_{I}\right) \\
& +w_{R} \cos \left(-\Omega t+n \theta+\Psi_{2}\right)+W_{I} \sin \left(-\Omega t+n \theta+\Psi_{2}\right) \\
u(x, \theta, t)= & U_{R} \cos \left(\Omega t+n \theta+\Psi_{I}\right)-U_{I} \sin \left(\Omega t+n \theta+\Psi_{I}\right) \\
& +U_{R} \cos \left(-\Omega t+n \theta+\Psi_{2}\right)+U_{I} \sin \left(-\Omega t+n \theta+\Psi_{2}\right) \\
v(x, \theta, t)= & v_{R} \cos \left(\Omega t+n \theta+\Psi_{I}\right)-V_{I} \sin \left(\Omega t+n \theta+\Psi_{I}\right) \\
& -v_{R} \cos \left(-\Omega t+n \theta+\Psi_{2}\right)-V_{I} \sin \left(-\Omega t+n \theta+\Psi_{2}\right)
\end{aligned}
$$

To eliminate the phase angles $\Psi_{1}$ and $\Psi_{2}$, we use the following change of variables:

$$
\Omega T=\Omega t+\frac{\Psi_{1}-\Psi_{2}}{2} \text { and } n \Theta=n \theta+\frac{\Psi_{1}+\Psi_{2}}{2}
$$

we then have

$$
\begin{aligned}
& w(x, \theta, t)=2\left(w_{R} \cos \Omega T-W_{I} \sin \Omega T\right) \cos n \Theta, \\
& u(x, \theta, t)=2\left(U_{R} \cos \Omega T-U_{I} \sin \Omega T\right) \cos n \Theta, \\
& v(x, \theta, t)=-2\left(v_{R} \sin \Omega T-V_{I} \cos \Omega T\right) \sin n \Theta,
\end{aligned}
$$

If we take the imaginary part rather than the real part, we simply change $[\omega T+n \Theta]$ to $\left[\omega T+n \Theta-\frac{\pi}{2}\right]$. The solution of the boundary-condition equations provides the functions $W(x)=w_{R}+i w_{Y}, U(x)=U_{R}+i U_{Y}$ and $V(x)=V_{R}+i V_{Y}$ and the preceding relations define the modal shapes. The displacements $u, v$ and $w$ are plotted versus the length of the cylinder, over half a period; namely, at times $t_{1}$, $t_{1}+\frac{T}{12}, t_{1}+\frac{T}{6}, \ldots . ., t_{1}+\frac{T}{2} \cdot$ They are normalized with respect to $x, \theta$ and $t$, according to 
- DB -

$$
\int_{0}^{l} \int_{0}^{2 \pi} \int_{0}^{T}\left[\frac{w(x, \theta, t)}{K}\right]^{2} d x d \theta d t=1
$$

where the coefficient $\mathrm{K}$ appears to be defined as

$$
k^{2}=2 \pi \int_{0}^{l}\left[w_{R}^{2}(x)+w_{I}^{2}(x)\right] d x
$$




\section{APPENDIX E \\ NUMERICAL METHOD - PROGRAM SAMPLE}

The computation was carried out with the aid of the 360/75 IBM Computer of the MCGill Computing Centre.

As an example, we briefly describe a computer program which has been written for the calculation of the frequencies of a clamped-Iree shell conveying fluid; the numerical results are presented in figure 19. The program consists of the MAIN with the following subprograms:

$\begin{array}{ll}\text { function } & \text { CFREQ, } \\ \text { function } & \text { CDET, } \\ \text { function } & \text { CDLA, } \\ \text { subroutine } & \text { BESSEL. }\end{array}$

All calculations are carried out with double precision. The MAIN program reads the data and predicts the points on the curve $\Omega(\bar{U})$ using a three points prediction formula.

The function subprogram CFREQ calculates the frequency in the range of two initial frequencies (CX, CY) and corresponding to a particular velocity UBAR. For a given frequency, COM, the eight characteristic roots, $\operatorname{CLAM}(I), I=1,2, \ldots, 8$, are determined, using a classical secant method, and classified. Then the corres- 
ponding parameters, CALPHA(I) and CBETA(I) are calculated and the boundary conditions are applied to define the determinant of the frequency equation. As this determinant introduces exponential terms of large positive argument and also of large negative argument, great care was taken to rearrange it and obtain meaningful results.

The function subprogram CDET calculates the determinant of the frequency equation. The method used is basically the Gauss-Jordan reduction where the largest eligible term in the pivotal column is selected. The function subprogram CDIA defines and calcu- . lates the determinant of the characteristic equation; as this determinant is of order three, a direct expansion is used.

The subroutine BESSEL calculates the modified Bessel functions of first kind, CBESI, and second kind, CBESK, with complex argument CLA. These functions are defined by series where a sufficient number of terms is retained to ensure a good accuracy. 
IMPLICIT COMPLEX $10(C), R E A L * 8(A, B, D-H, D-Z)$

COMMDN CI, ANU, ANUM, ANUP, A, AL, AK, UBAR,EPSI, STEP, IN,L CUMMON/BODY/NC

C

OJMENSIUN CAM $(60)$, DEL $(60)$

\section{INPUT DATA}

c

KEAD $(5,1000) A, A L, H, E, A N U, R O S, N C$

1000 FURMAT $(6010,3$, I5)

WRITE(6,1100) A, AL,H,E, ANU, RUS, NC

1100 FURMAT $(6012.5,15 / 1)$

$\bar{A} K=H \neq H / 12,000 / A / A$

ANUM $=(1.000-A N U) / 2,000$

ANUP $=(1,000+$ ANU $) / 2,000$

$\bar{C} I=\{0,000,1,000\}$

EPS $1=6,0-02$

$L=1$

$U B A R=0,50$

STEP $=0,0500$

C

C

STARTING POINTS FUR THE CALCULATIIN UF FREQUENCJES

Cl= INITIAL VALUE UF FRFQUENCY

C $2=$ UIFFERENT INITIAL VALUE OF FREQUENCY

CAIN $(1)=C F K E Q(C, 1, C 2)$

UBAR $=U B A R+S T E P$

C $1=C A M(1)$

$C Z=C A H(1)+(0,00,0,2000-03)$

CAM(2) =CFKEQ(CI,C2)

UEL $(2)=S T E P$

$\cup B A R=U B A R+S T E P$

$C 1=C A H(2)$

$C 2=C A H(2)+(0,00,0.200 D-03)$

CAM (3) =CFREQ $\left(C_{1}, C_{2}\right)$

UEL $(3)=$ STEP

IP $=4$

$10 \cup B A R=U B A R+S T E P$

UEL $(I P)=S T E P$

$C I=C A H(I P-1)+C E L(I P) / D E L(I P-2) *((D E L(I P)+O E L(I P-1)+(J F L(I P-2))$

1/DEL(IP-I)* $(C A M(I P-1)-C A M(I P-2))+(D E L(I P)+D E L(I P-1))$

$2 i(D E L(I P-1)+U E L(I P-2)) *(C A H(I P-3)-C A H(I P-1)))$

$C 2=(1+(0,10-04,0,10-04)$

$C A M(I P)=C F R E O(C 1, C 2)$

IF(IN.EQ, 10.AND.IF,LT.4) GO TG jGO

IF (IN,EQ, IO) G.L TO 10

$1 P=1 P+1$

IF(IP.ER, 22) R, TL 360

iSL TO 10

$3603 T$ STP

END 
CUMPLEX FUNCTIDIN CFREQ* I6(CX,CY)

IMPLICIT COMPLEX* LO (C),REAL $\$ 3(A, B, D-H, D-Z)$

CUMMON CI, ANU, AN'JM, ANUP, A, AL, AK, IJBAR, EPSI, STEP, IN,L

COMMON/BOUY/NC̈

COMMON/ IHIJLD/CLAT $(20), C D E L T A, C L I 1, K J U V T$

DIMENSIUN CLA (40), CDEL (40), CMMEGA $(40), C V(40), C B A(32), C L A M(8)$,

ICO(9), CALPHA ( 8$), C B E T A(8), C A(8,8)$

CUMEGA $(1)=C X$

CUMEGA $(2)=C Y$

C

IF(L,NE,I) GI] TU 410

C STARTING POINTS FUR THE CALCULATIUH OF LAMBDAS

C.

$C B A(1)=(0.54000,0.72000)$

$C B A(2)=(0.55000,0.73000)$

$C B A(3)=(0.34000,-0.72000)$

$C B A(4)=(0.55000,-0.73000)$

$C B A(5)=(-0.54000,0.72000)$

$C B A(6)=(-0,55000,0,73000)$

$C B A(7)=(-0,54000,-0.72000)$

$C B A(8)=(-0,55000,-0,73000)$

CBA $(9)=(0,7900+01,0.8400+01)$

$C B A(10)=(0.8000+01,0.35011+01)$

$C B A(11)=\{0,7900+01,-0,8400+01\}$

$C B A(12)=(0,8000+01,-0,8500+01)$

$C B A(13)=(-0,7901)+01,0.8400+01)$

CBA $(14)=(-0,30001+01,0,8501)+01)$

$C B A(15)=(-0,790(1)+01,-0.3400+01)$

$C B A(16)=(-0.8000+01,-0.8500+01)$

C

$L=2$

CLMPUTATIUN DF LAMBDAS ( 8 ROUTSS)

$410 \quad I N=1$

400 CUM $=$ CDHEGA $(I N)$

CDELTA $=$ COM $\angle O M$

KUUNT $=1$

\& $\bar{K} L=2 * K U: J N T$

$C L A(1)=C B A(K L-1)$

$C L A(2)=C B A(K L)$

CUEL $(1)=C O L A(C L A(1))$

WRITE $(0,1004)$ CLAC 1$),$ CDEL (1)

CDEL $(2)=C O L A(C L A(2))$

WRIIE $(0,1004)$ CLA(2), CI)EL(2)

$\square 0=2$

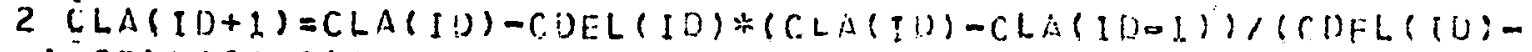

$1 \operatorname{CDE}((1 D-1))$

COEL $(1 D+1)=C D L A(C L A(I D+1))$

WRITE $(0,1004)$ CLA $(I 0+1)$, CI)EL(ID+1)

6

1004 FURHAT $(1 H, 62 X, 4015.8)$

C CHECK UF COMVEPGF:NCE

c

$X=C D E L(1 D+1)$ 


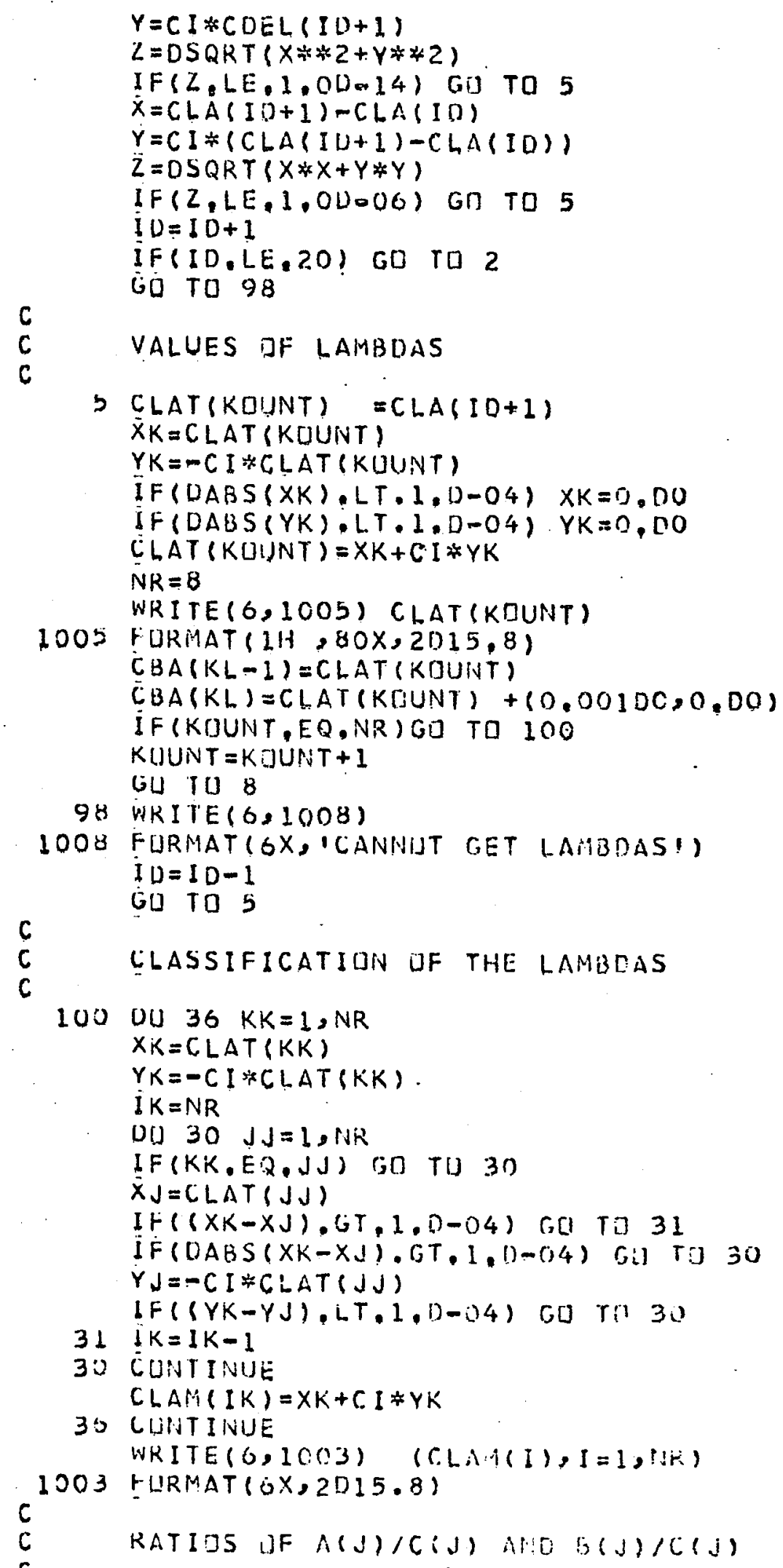




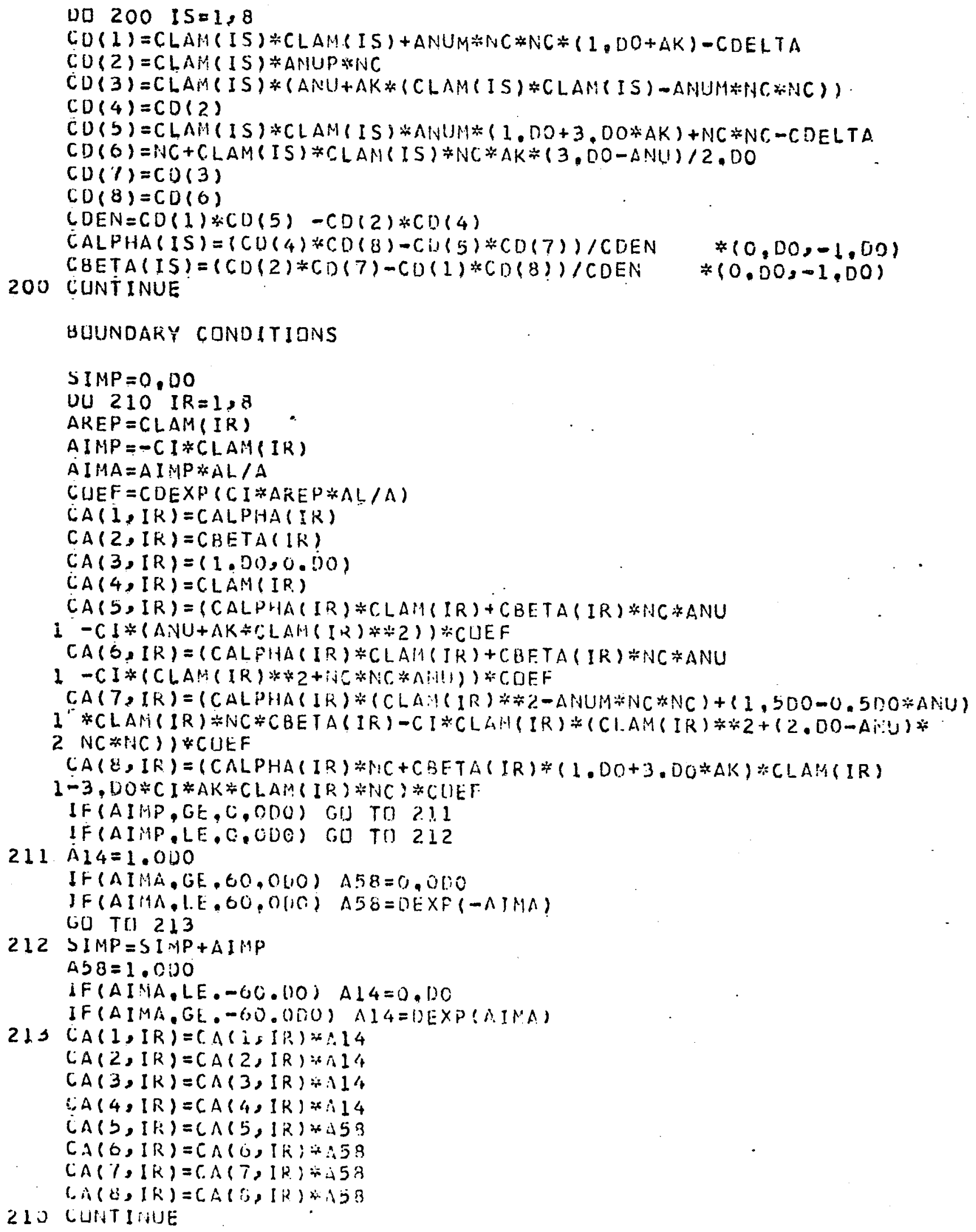


- E7 -

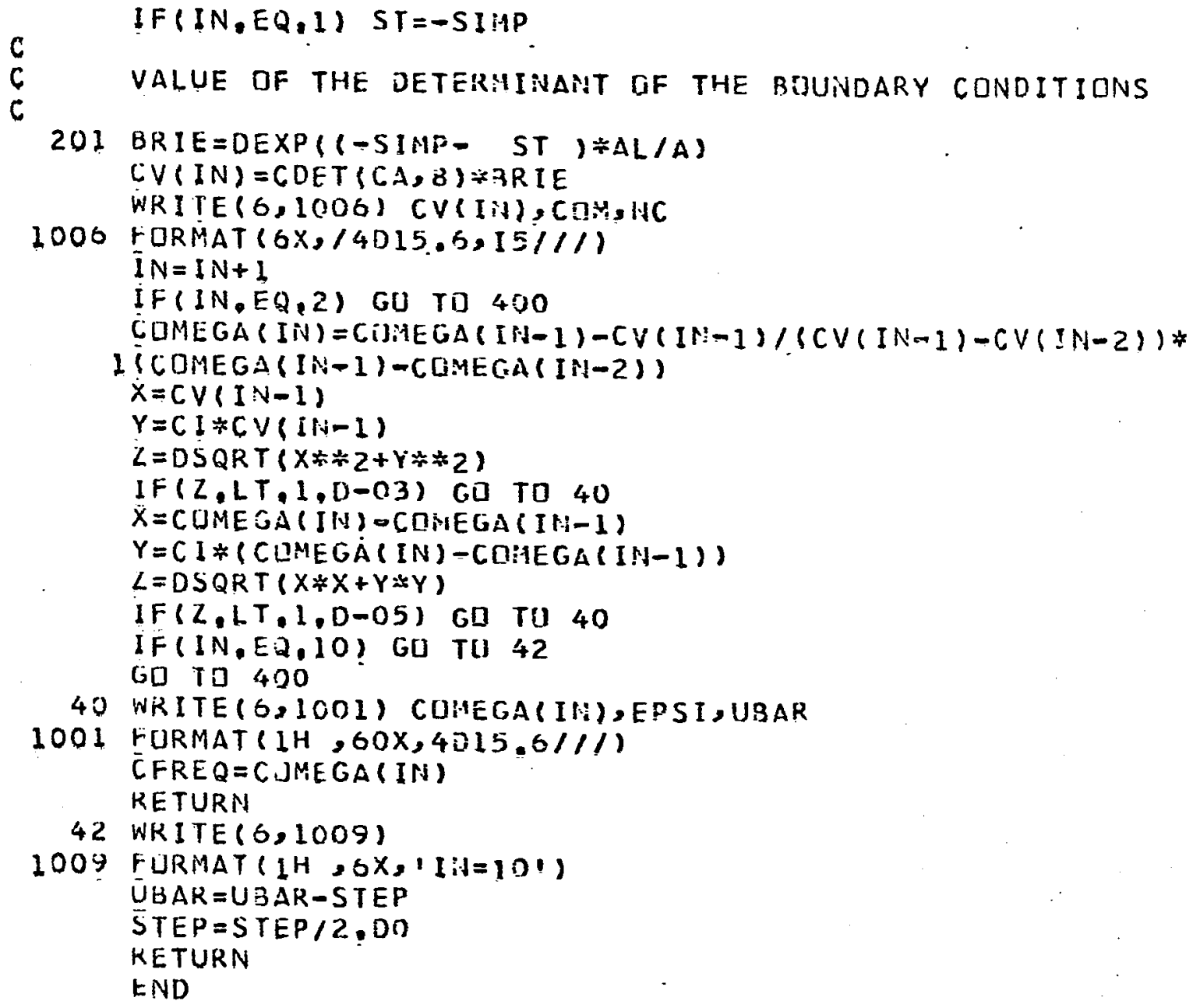




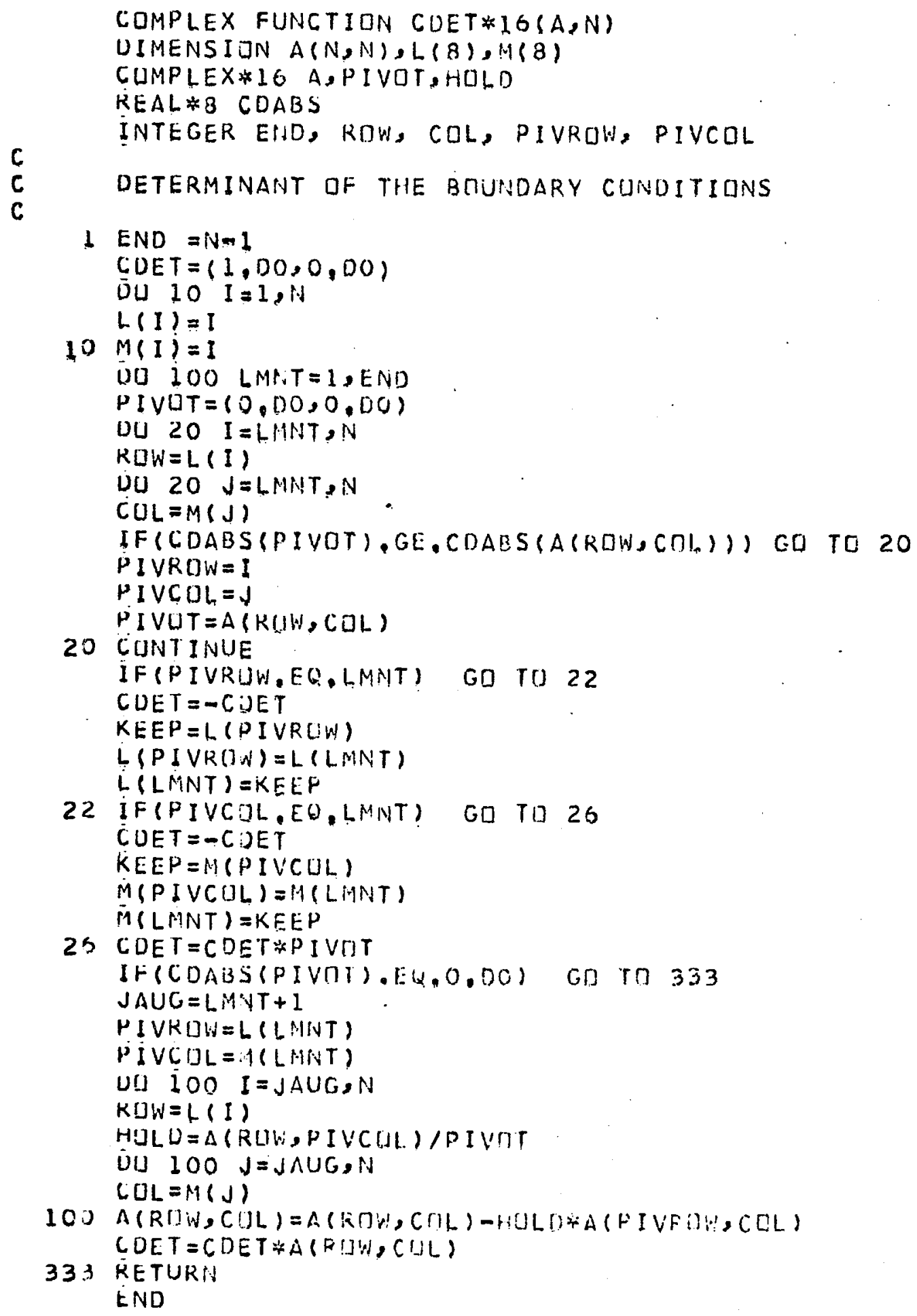


CDMPLEX FUNCTION COLA*16(CLA)

IMPLICIT CDMPLEX * $16(C), R E A L * 3(A, 6,0-H, D-Z)$

COMMON CI, ANU, NINUH, ANUP, A, AL, AK, LUBAR, EPSI, STEP, IN,L

CLMMON/BOOY/IC

COMMON/HOLD/CLAT $(20)$, CDELTA,CLM,KDUNT

DIMENS I UN CD(9)

DETERMINANT UF THE LAMBDAS

CALL BESSEL (CLA,CBESI, CBESK)

$C D(I)=C L A * C L A+A N U(A * N C * N C *(1,00+A K)-C D E L T A$

$C U(2)=C L A * A N U P * N C$

$C D(3)=C L A *(A N U+A K *(C L A * C L A-A N U N * N C * N C))$

C. $D(4)=C D(2)$

$C D(5)=C L A * C L A * A N U M *\{1, D O+3 . D O * A K)+N C * N C-C D E L T A$

$C O(G)=N C+C L A * C L A * C C * A K *(3, D O-A N C) / 2, D O$

$C D(7)=C D(3)$

$C 0(8)=C 0(6)$

$C D(9)=1, D O-C D E L T A+A K *((C L A * C(A+R C * N C) \neq 2-2, D \cap * N C * N C+1, D O)$

$1-E P S I *(C B E S I *(C U M+U B A R * C L A) * 2+C B E S K * C D E L T A)$

$C O L A=C D(1) * C D(5) * C D(9)+C D(2) * C D(6) * C D(7)+C D(3) * C D(4) * C D(8)$

$1-C D(1) * C D(6) * C D(8)-C D(2) * C 1)(4) * C D(9)-C D(3) * C D(5) * C D(7)$

IF (KOUNT,EQ,1) GO TO 2

$K M=K D \cup N T-1$

UU $4 \quad J=1, K M$

$4 C D L A=C D L A /(C L A-C L A T(J))$

2 RETURN

END 


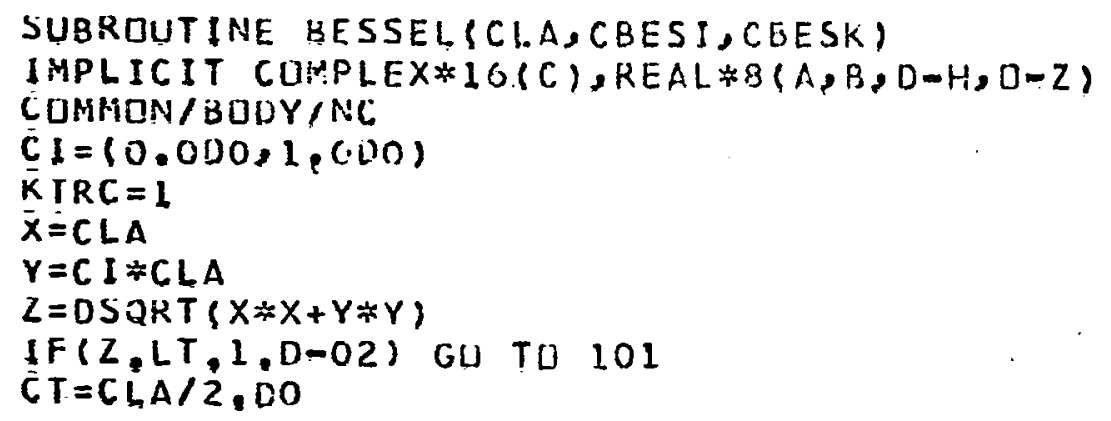

K BESSEL FUNCTIJH

$C Y Y=C D L U G(C T)+0.017215664901533$

$C Z Z=(1.000,0.000)$

IF(NiC,EQ, I) GU TO 100

UU 4 I $Y=2$, IiC

$C Y Y=C Y Y-0.500 / I Y$

$C Z Z=C Z Z / I Y$

4 CUNT INUE

100 CBESK $=C Y Y * C Z Z$

UU $5 \quad I Z=1,30$

$(Y Y=C Y Y-(1.00 / 1 Z+1,00 /(I Z+1, C)) / 2,00$

$C Z Z=C Z Z * C T \approx C T / I Z /(I Z+N C)$

$C Y Z=C Y Y * C Z Z$

$A=C Y Z$

$B=C I \neq C Y Z$

$Y L=U S A K T(A * A+B * B)$

IF(YZ.LE.1.OD-12) Gi] TO 20

CDESK $=$ C SESK + CYZ

$\checkmark$ CLIST I IJUE

20 CBESK $=C$ SESK $*(-1, r: 0) \div(M C+1) * C T \div C$

IF (1.C.E2, 1) CBESK=CBISK+1.00/2.UO/CT

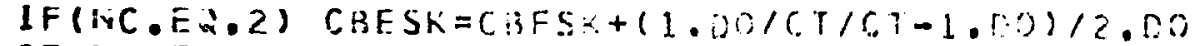

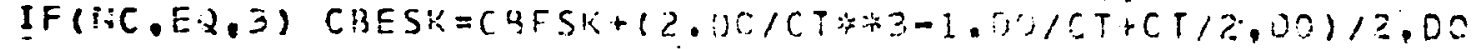


IF (KTRC,EQ,2) GD TD 6

$N C=N C+1$

$K T R C=K T R C+1$

$C B I=C B E S I$

$C B K=C B E S K$

Gid TO 10

6 $N C=N C-1$

CRATI $=N C+2, D O * C T * C B E S I / C B I$

CRATK $=I N C-2, D O * C T * C B E S K / C B K$

CBESI $=1,00 / C R A T I$

CBESK $=1$, DO/CRATK

RETURN

$101 \mathrm{CBESI}=1, \mathrm{DO} / \mathrm{NC}$

CBESK $=-1,00 / N C$

RETURN

END 
PHOTOGRAPHS AND FIGURES 

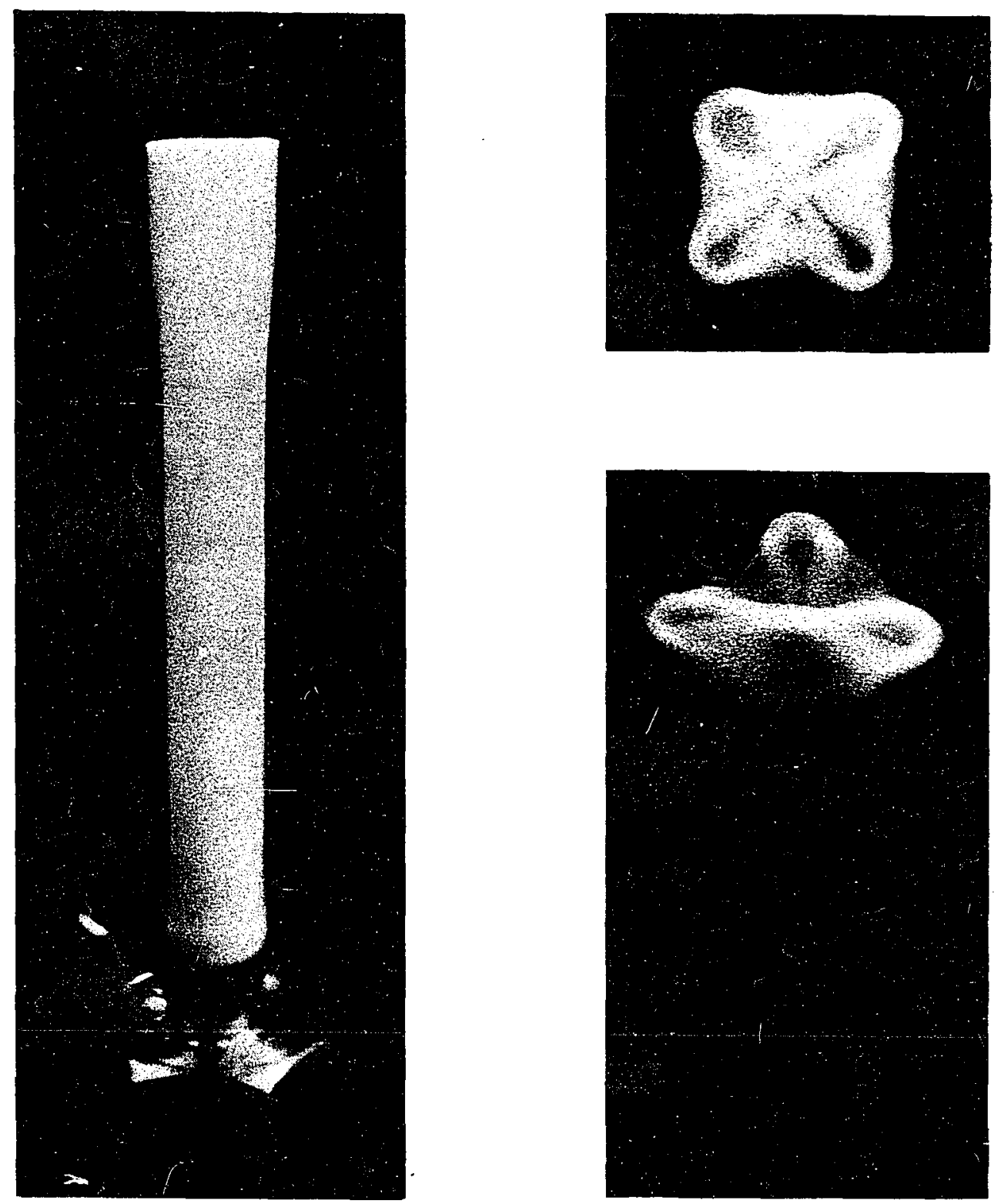

Photographs showing the flutter of a silastic clamped-free tube ( $a=0.28 \mathrm{in}, \mathrm{h}=0.06 \mathrm{in}$, shutter-speed $=1 / 500)$ 


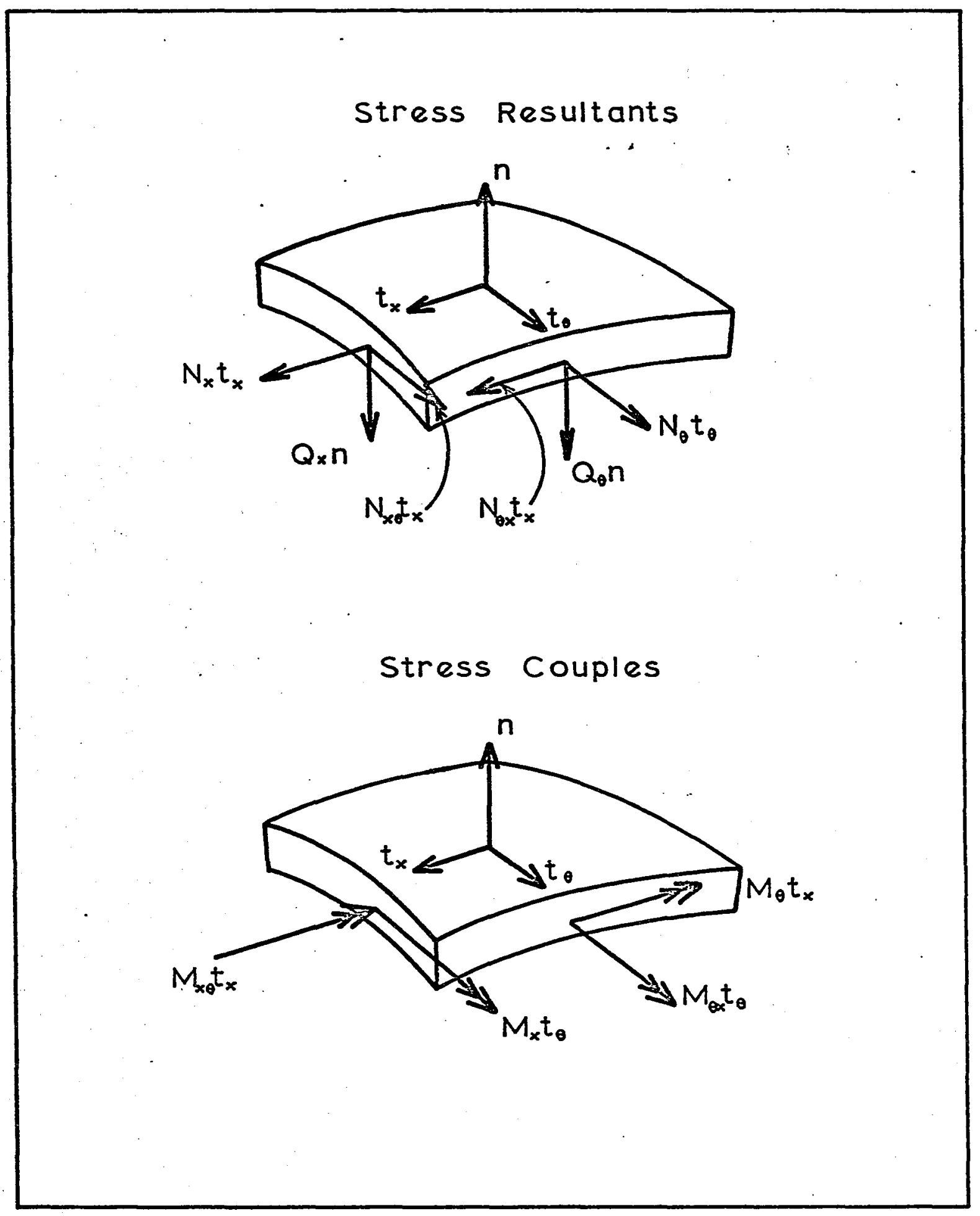

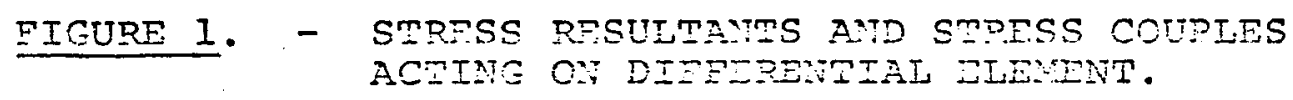




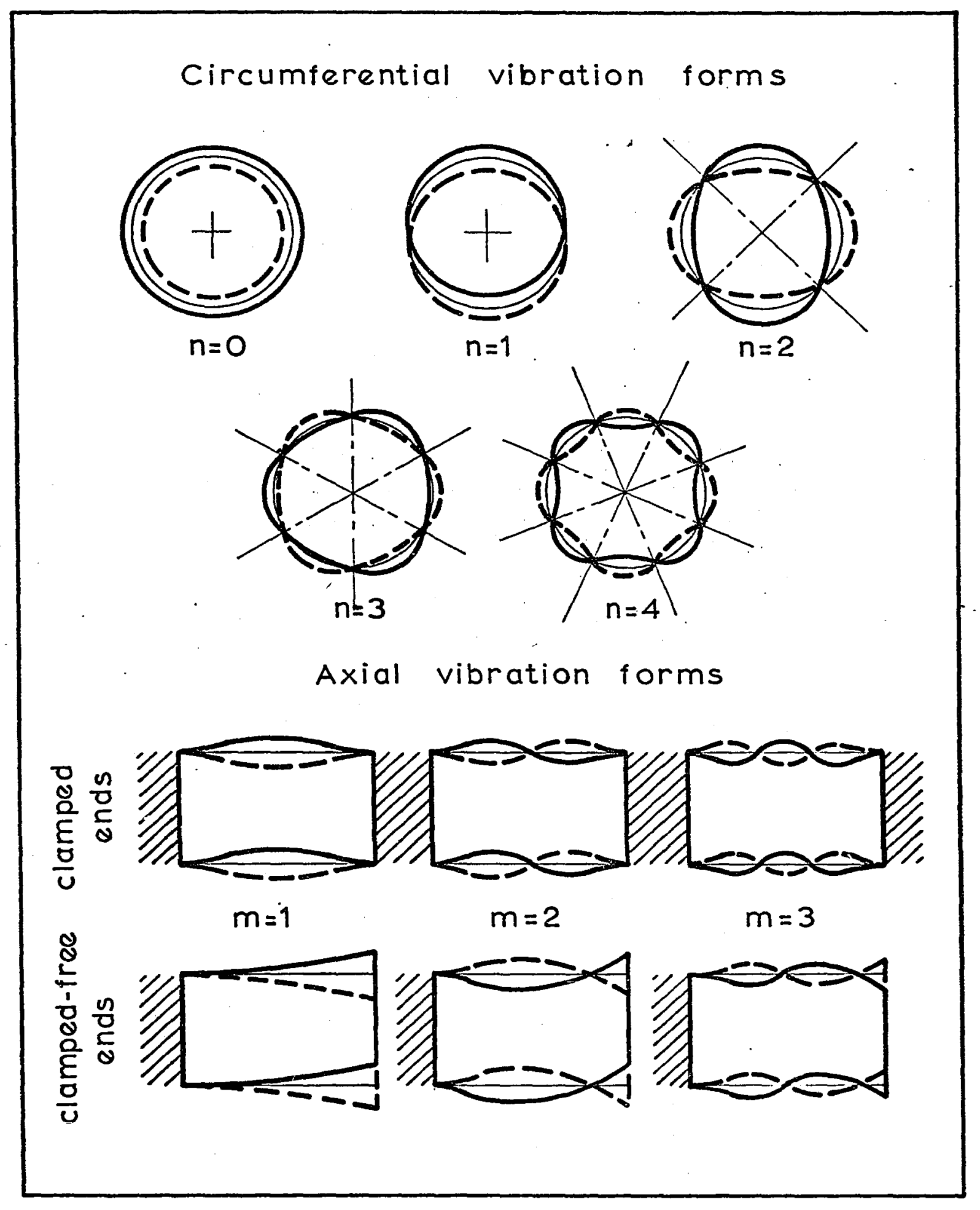

FIGURE 2. - VIBRATIOA FOPUS FOR CIRCUEAR CVIIXIPICAL SEELIS. 


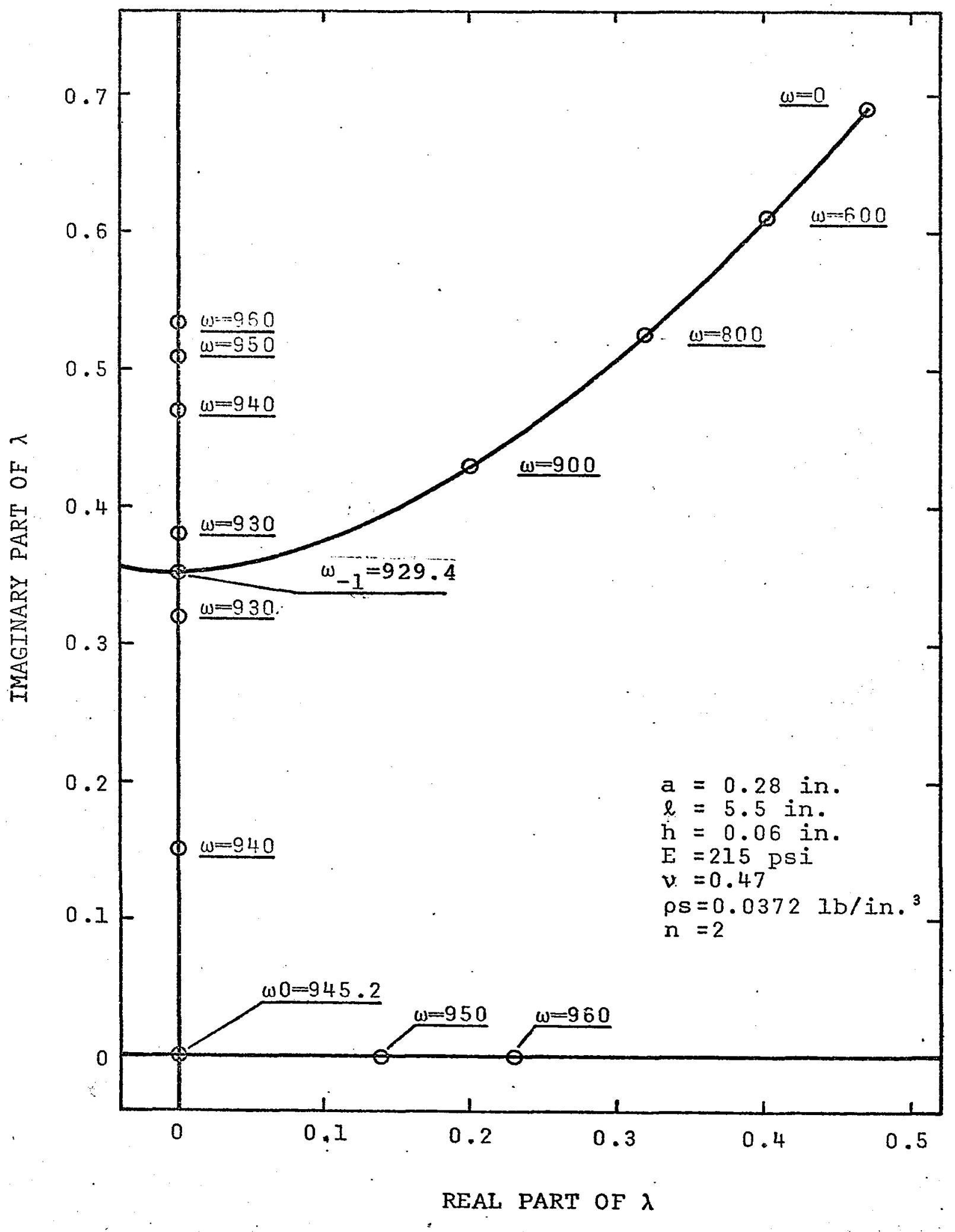

FIGURE 3. - TYPICAL ARGAND DIAGPAM OF WAVE. NUMBER, $\lambda$, AS FUNCTION OF FREQUENCY, FOR A SILASTIC TUBE WITH BOTH ENDS CLAMPED $(n=2)$. 


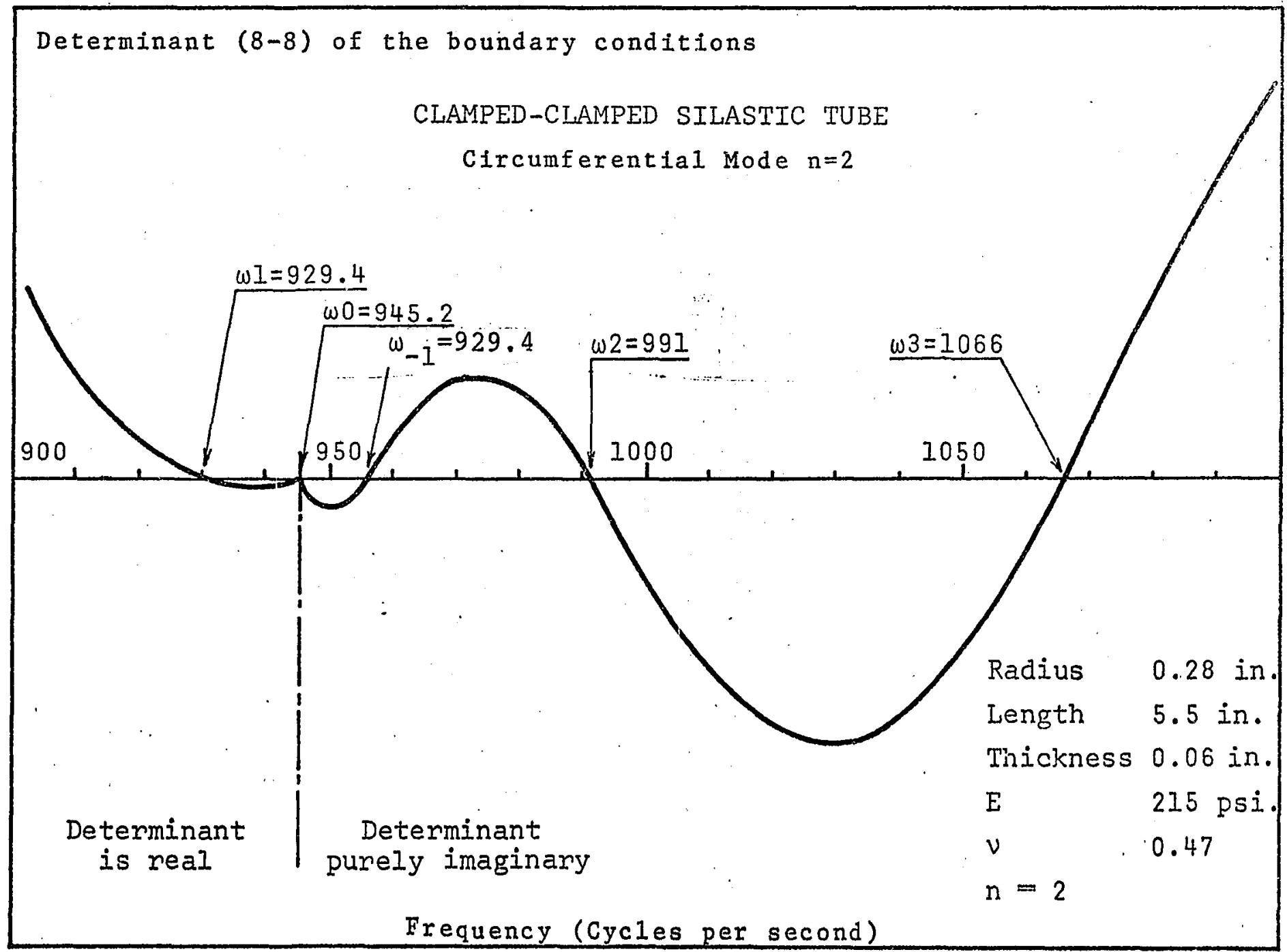

FIGURE 4, - VARIATION OE THE DETERMINANT OF THE BOUNDARY CONDITIONS, $\triangle$, VERSUS CIRCULAR FREQUENCY, FOR A SILASTIC TUBE WITH BOTH ENDS CLAMPED $(n=2)$. 


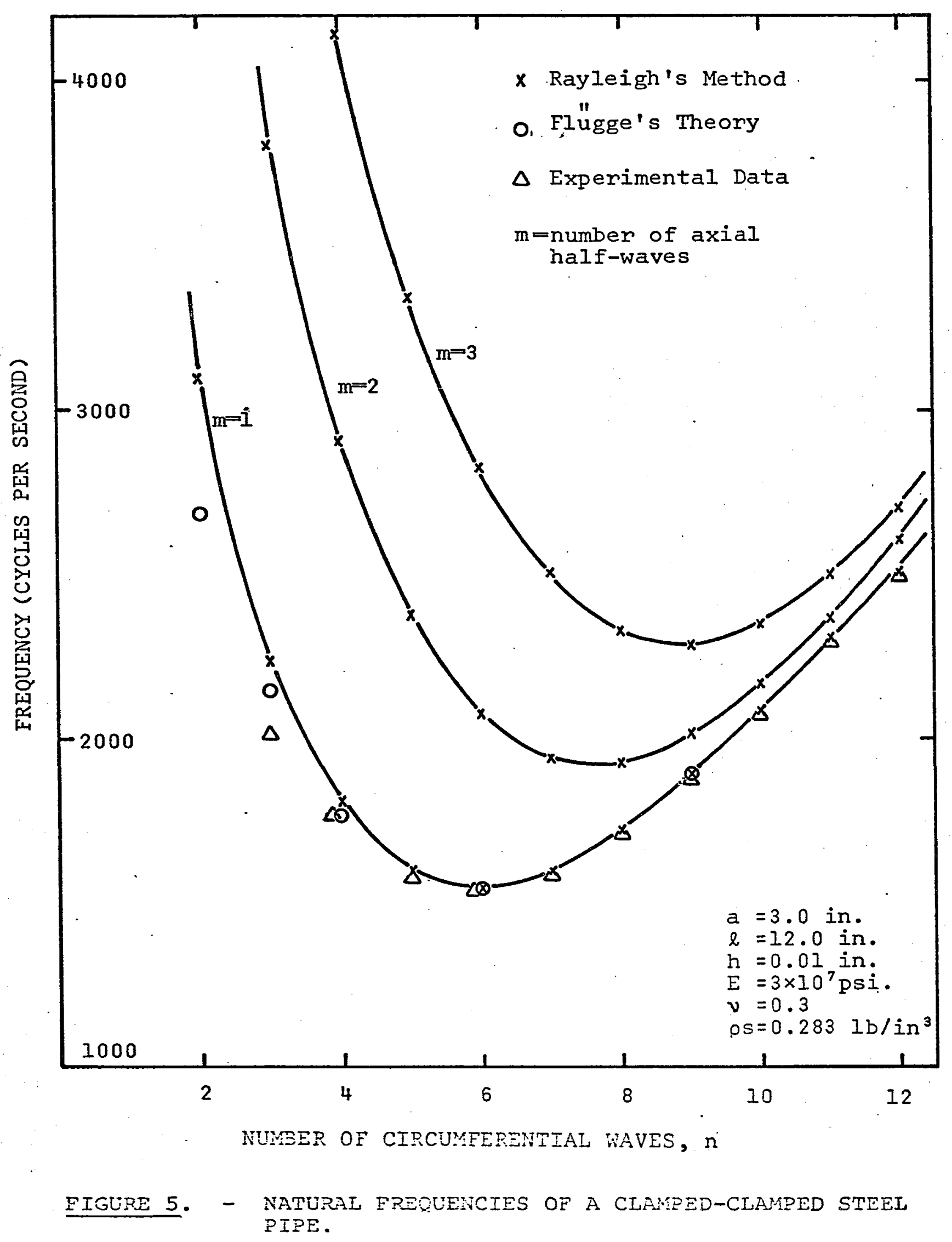




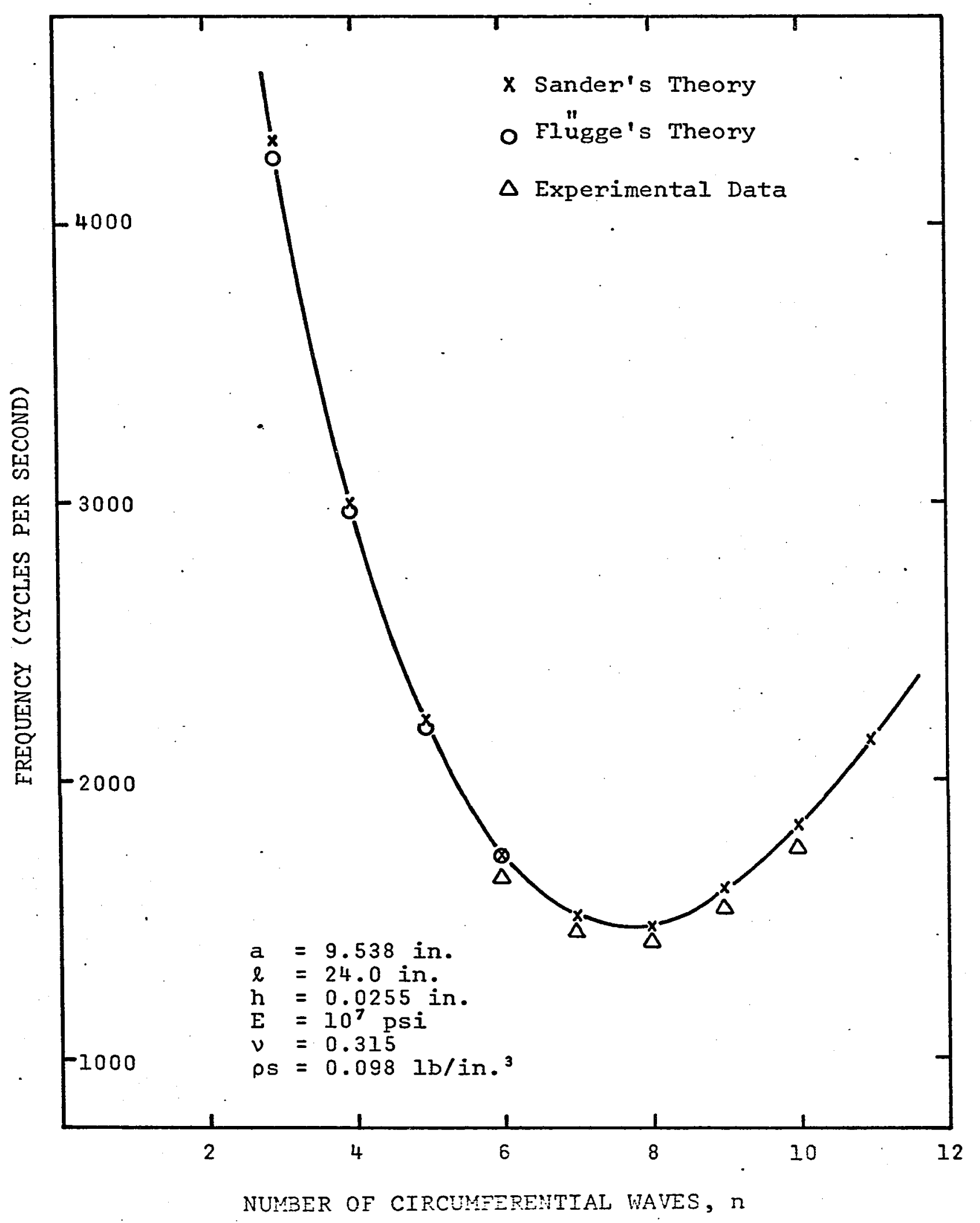

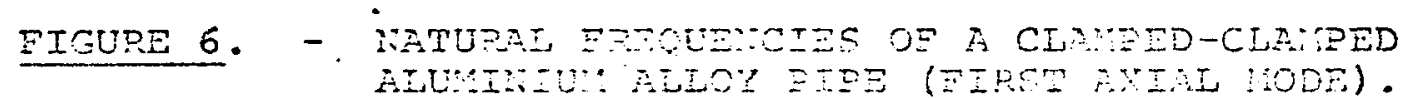


FIGURE 7 - Modal shapes for the first three axial modes of a clamped-clamped rubber tube.

$$
\begin{aligned}
& \mathrm{h} / \mathrm{a}=2.27 \times 10^{-2} \\
& \ell / a=25.9 \\
& \nu \quad=0.50 \\
& \mathrm{n}=2
\end{aligned}
$$



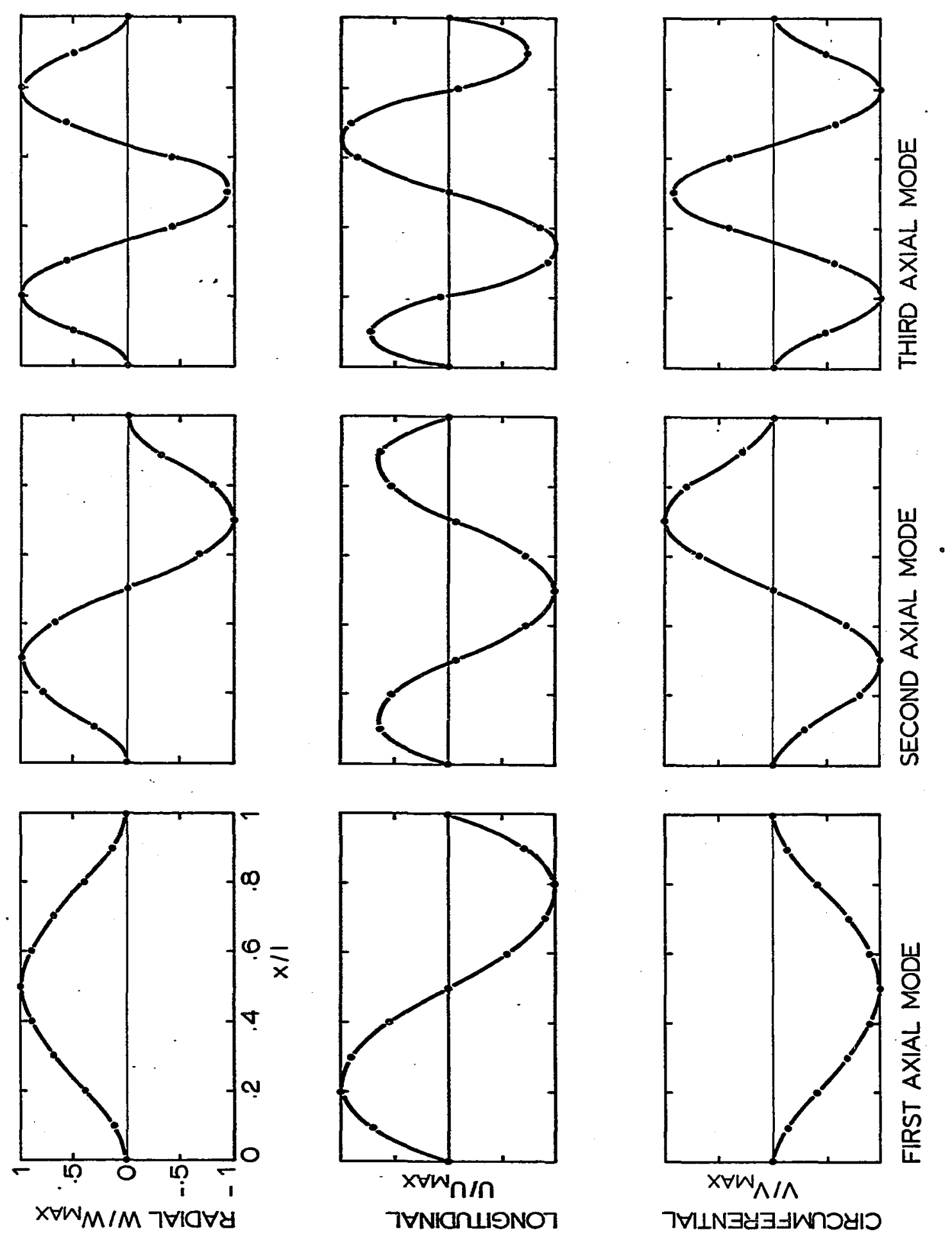


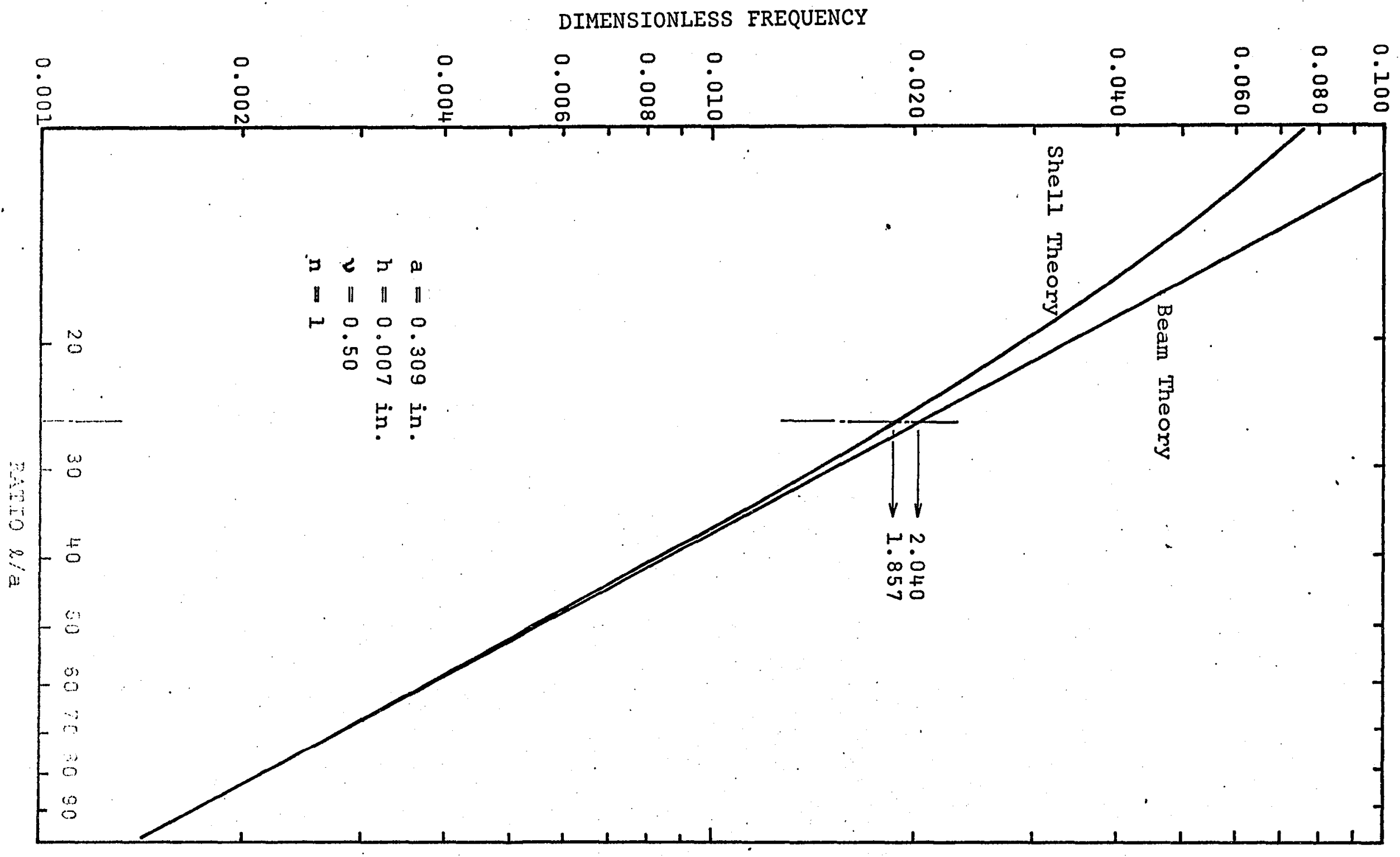

FIGURE 8. - COMPARISON OF SHTLI AND BEAM THEORIES: NATURAL FREQUENCIES OF A CLAMPED-CLAMPED TUBE $(n=1)$. 


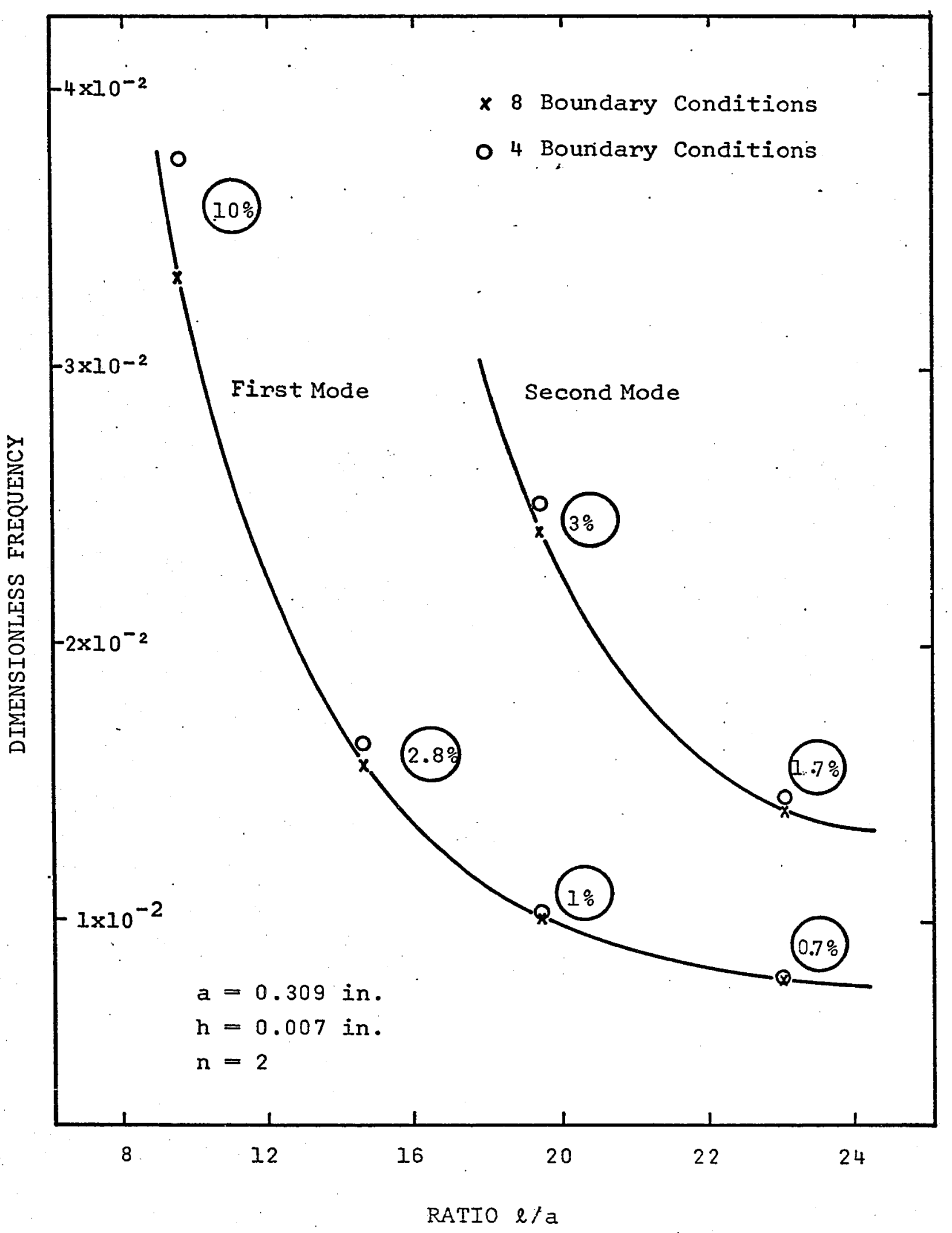

FIGURE 9.- INELUENCE OF EONNDAPY CONDITIONS USED IN THE
ANALYSIS ON THE NATUPAI FPIQUENCIES OE A CLAMPED-
CLAMPED TUBE $(\mathrm{n}=2)$. 


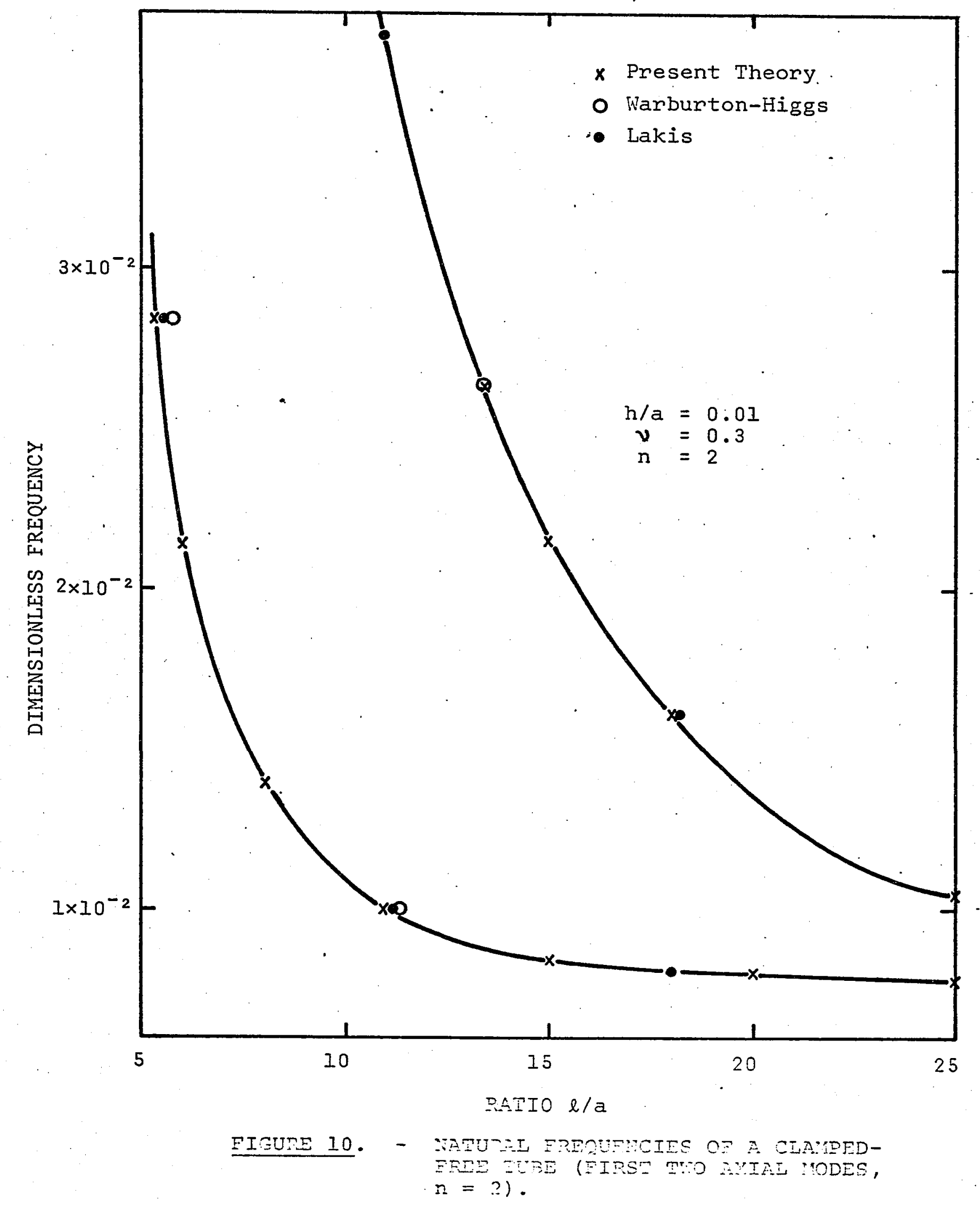




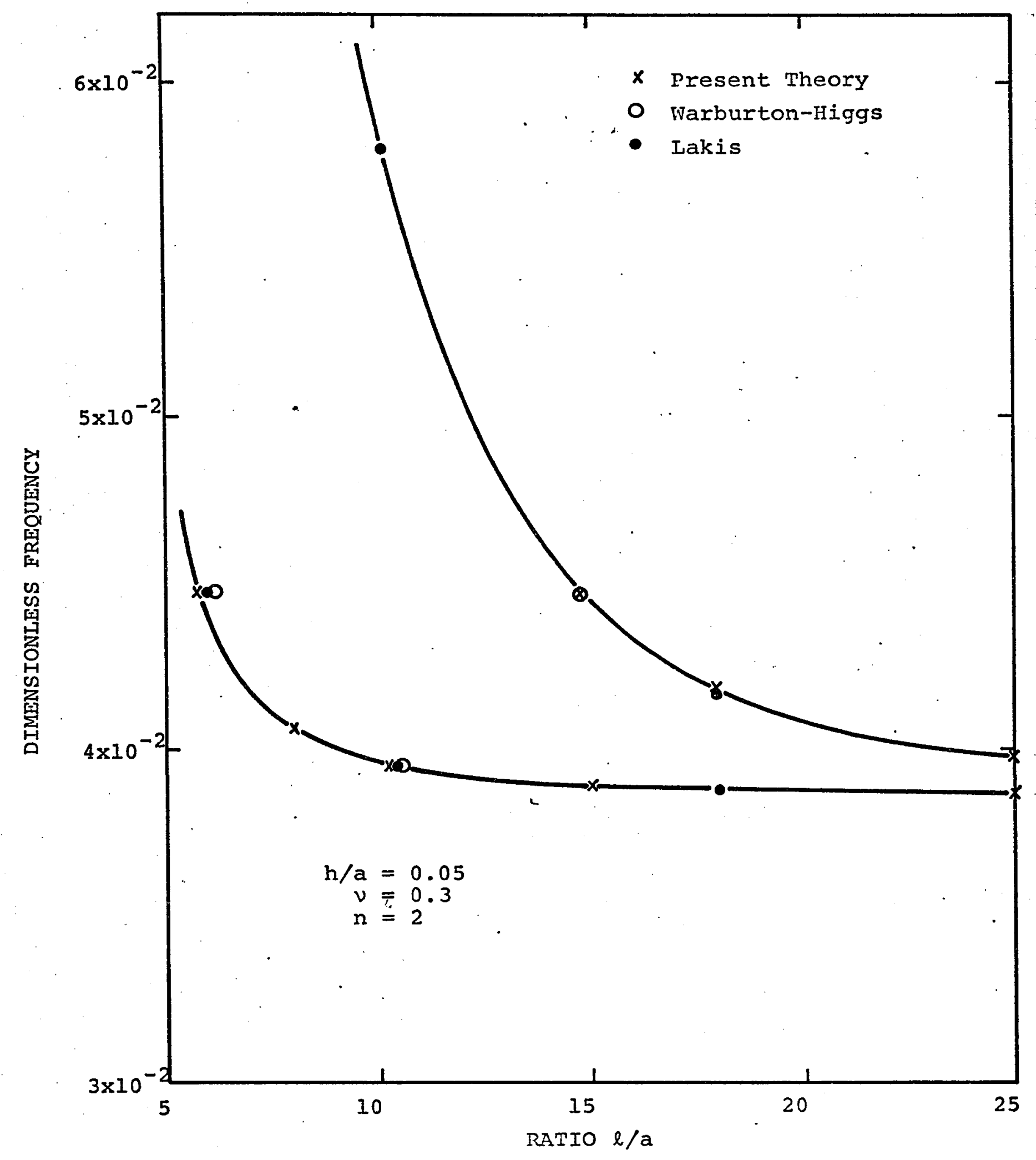

FIGURE 11. - MATURA EREOUDTCIES OP A CIAVDED-FREE TUBE (ETPSZ MYO RYAL MODES, $\mathrm{n}=2$ ) 
FIGURE 12 - Modal shapes for the first two axial modes of a clamped-free tube.

$$
\begin{aligned}
& \mathrm{h} / \mathrm{a}=5 \times 10^{-2} \\
& \ell / \mathrm{a}=10.0 \\
& \nu=0.30 \\
& \mathrm{n}=2
\end{aligned}
$$




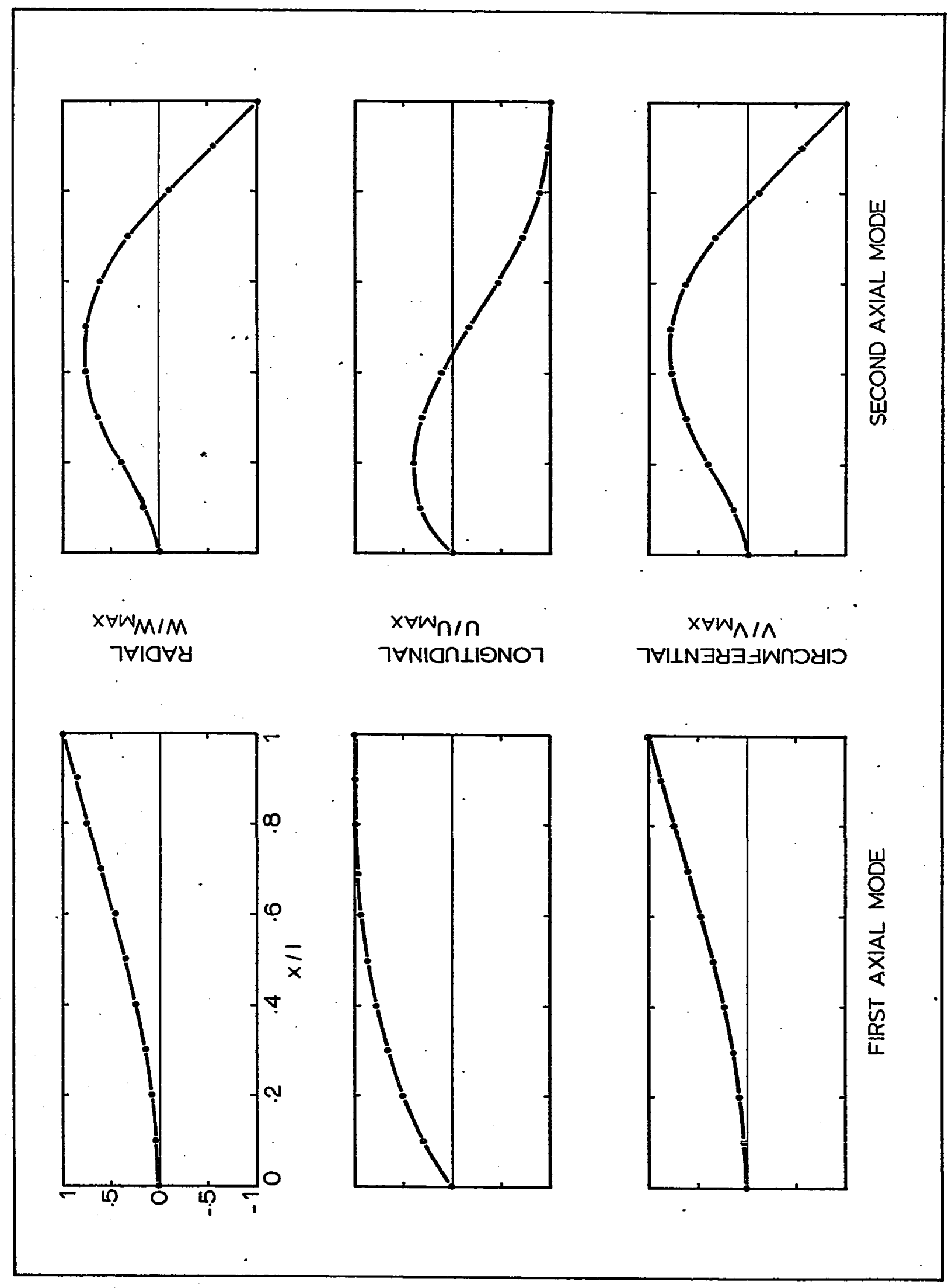


FIGURE 13 - Variation of dimensionless frequency, $\Omega$, with dimensionless velocity, $\bar{U}$, for the first three axial modes of a clamped-clamped rubber tube.

$$
\begin{aligned}
& \mathrm{h} / \mathrm{a}=2.27 \times 10^{-2} \\
& \ell / \mathrm{a}=25.9 \\
& \nu \quad=0.50 \\
& \mathrm{n}=2
\end{aligned}
$$




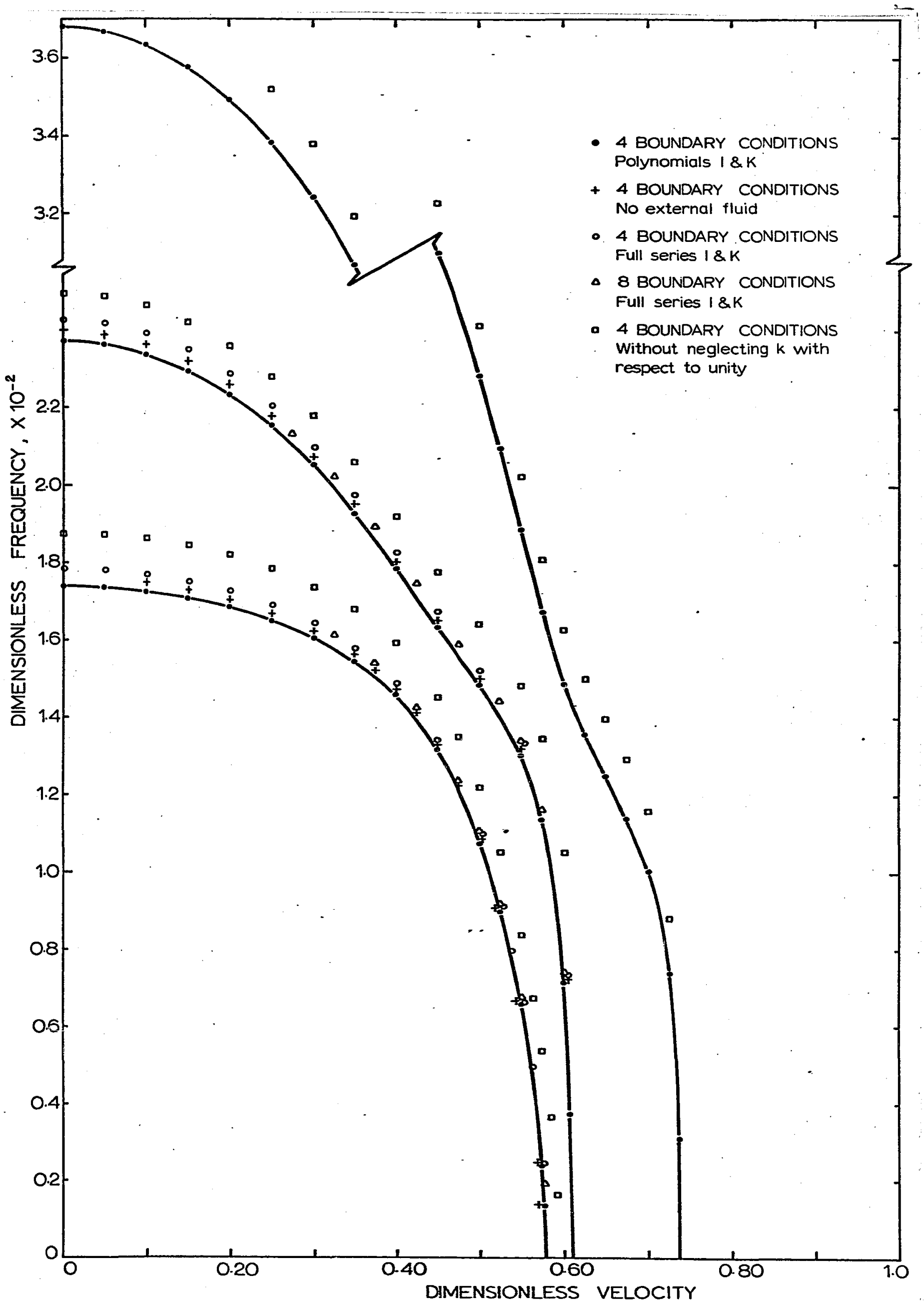




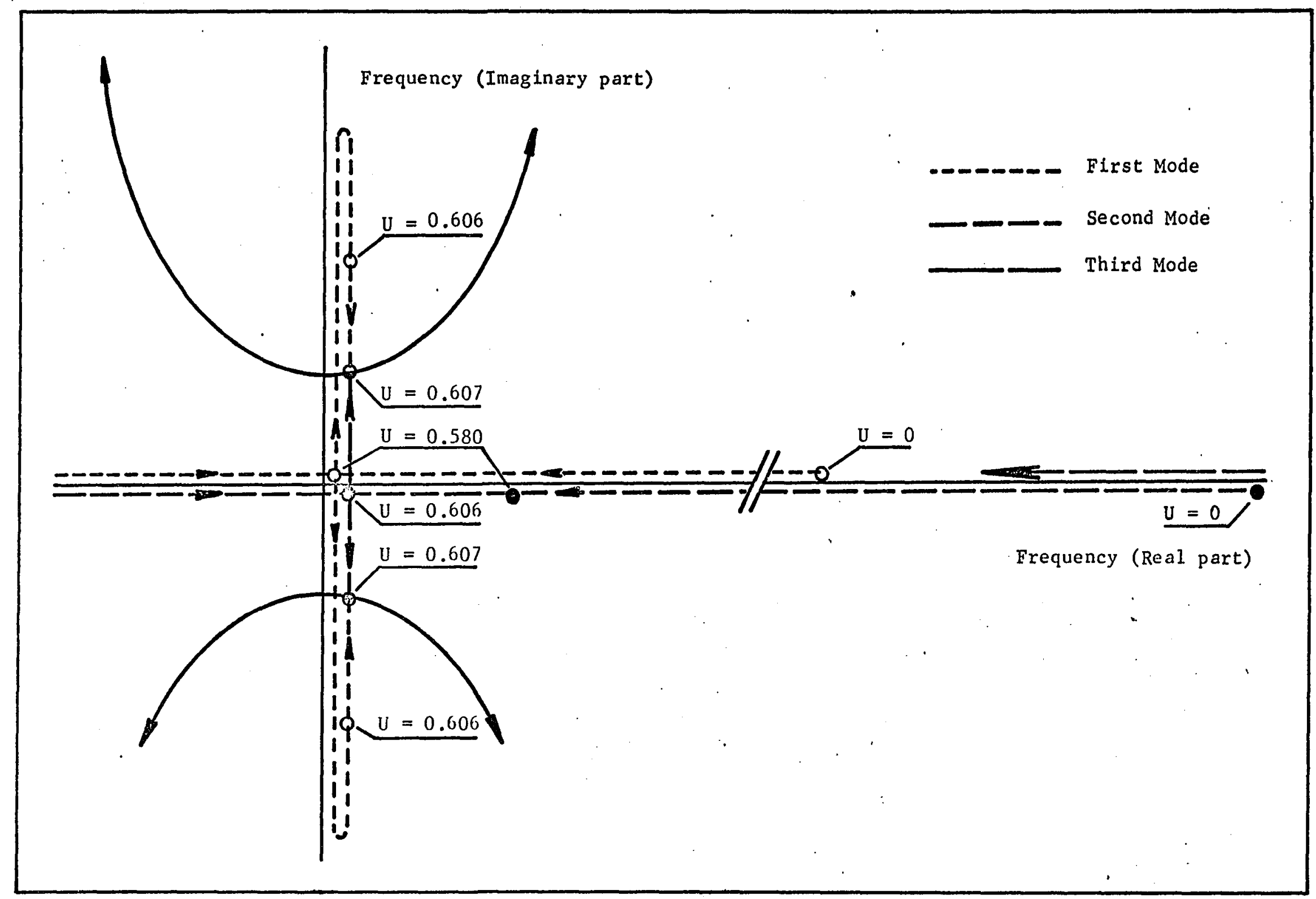

FIGURE 14. - TYPICAI ARGAND DIAGRAM OF DIMENSIONLESS FREQUENCY, $\Omega$, AS FUNCTION OF DIMENSIONLESS FLOW VELOCITY, $\bar{U}$, FOR A CLAMPED-CLAMPED TUBE $\left(\mathrm{h} / \mathrm{a}=2.3 \times 10^{-2}, \mathrm{l} / \mathrm{a}=26\right)$. THE LOCI ON THE REAL AND IMAGINARY AXES HAVE BEEN DRAWN OFF THE AXES BUT PARALLEL TO THEM, FOR CLARITY. 
FIGURE 15 - Variation of dimensionless frequency, $\Omega$, with dimensionless velocity, $\bar{U}$, for the first three circumferential modes of a clamped-clamped rubber tube (axial modes, $m=1,2$, also $m=3$ for $n=2$ ).

$$
\begin{aligned}
h / a & =2.27 \times 10^{-2} \\
\ell / a & =25.9 \\
\nu & =0.50 \\
n & =1,2 \text { and } 3 \\
m & =1,2 \text { and } 3
\end{aligned}
$$




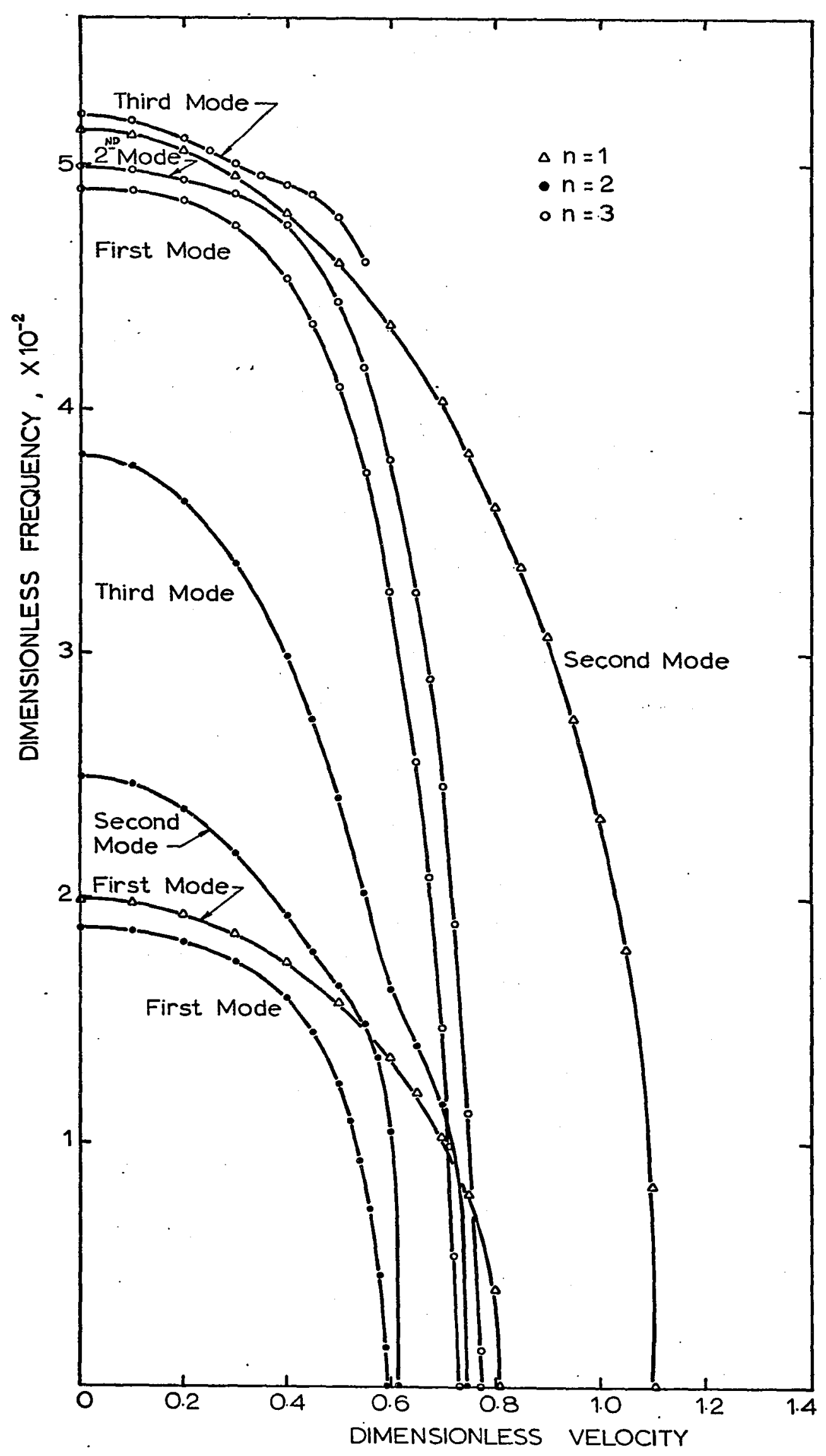


FIGURE 16 - Variation of dimensionless buckling velocity, $\overline{\mathrm{U}}_{\mathrm{B}}$, with length/radius ratio, for the first three circumferential modes of a clamped-clamped rubber tube (axial modes,

$$
\begin{aligned}
\mathrm{m}=1,2, \text { also } \mathrm{m}= & 3 \text { for } \mathrm{n}=2 \text { ). } \\
\mathrm{h} / \mathrm{a} & =2.2710^{-2} \\
\ell / a & =25.9 \\
v & =0.50 \\
\mathrm{n} & =1,2 \text { and } 3 \\
\mathrm{~m} & =1,2 \text { and } 3
\end{aligned}
$$




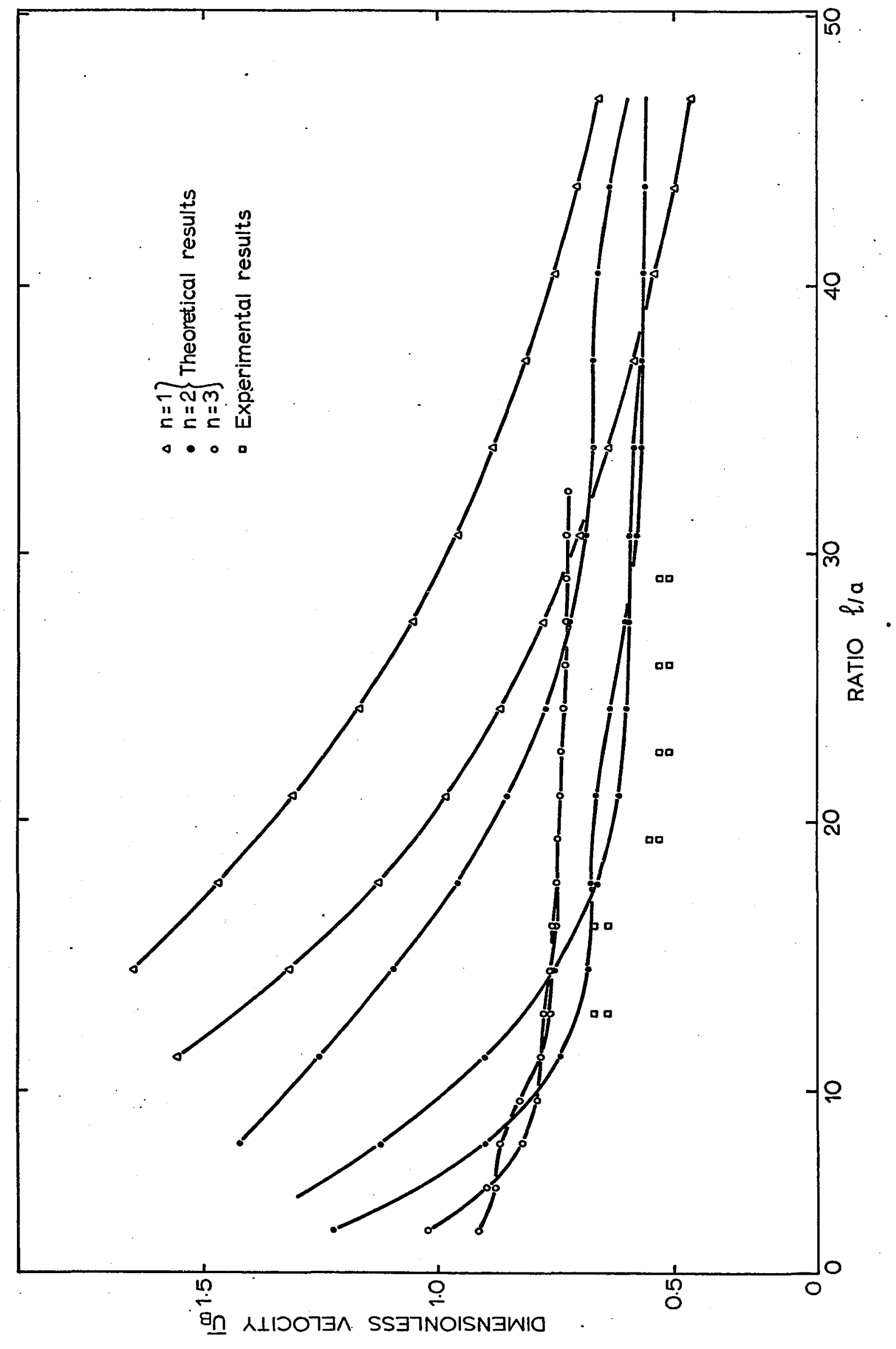


FIGURES $17 a, b, c, d, e$ - Time-dependence of modal shapes over half a period, as function of dimensionless velocity, $\bar{U}$. First axial mode at zero velocity of a clamped-clamped rubber tube.

$$
\begin{aligned}
& \mathrm{h} / \mathrm{a}=2.27 \times 10^{-2} \\
& \ell / \mathrm{a}=25.9 \\
& \nu=0.50 \\
& \mathrm{n}=2
\end{aligned}
$$


CIRCUMFERENT IAL WAVE NUMBER $\mathrm{N}=2$

NUMBER OF AXIAL HALF WAVES $M=1$

DIMENSIONLESS VELOCITY $\bar{U}=0.0$

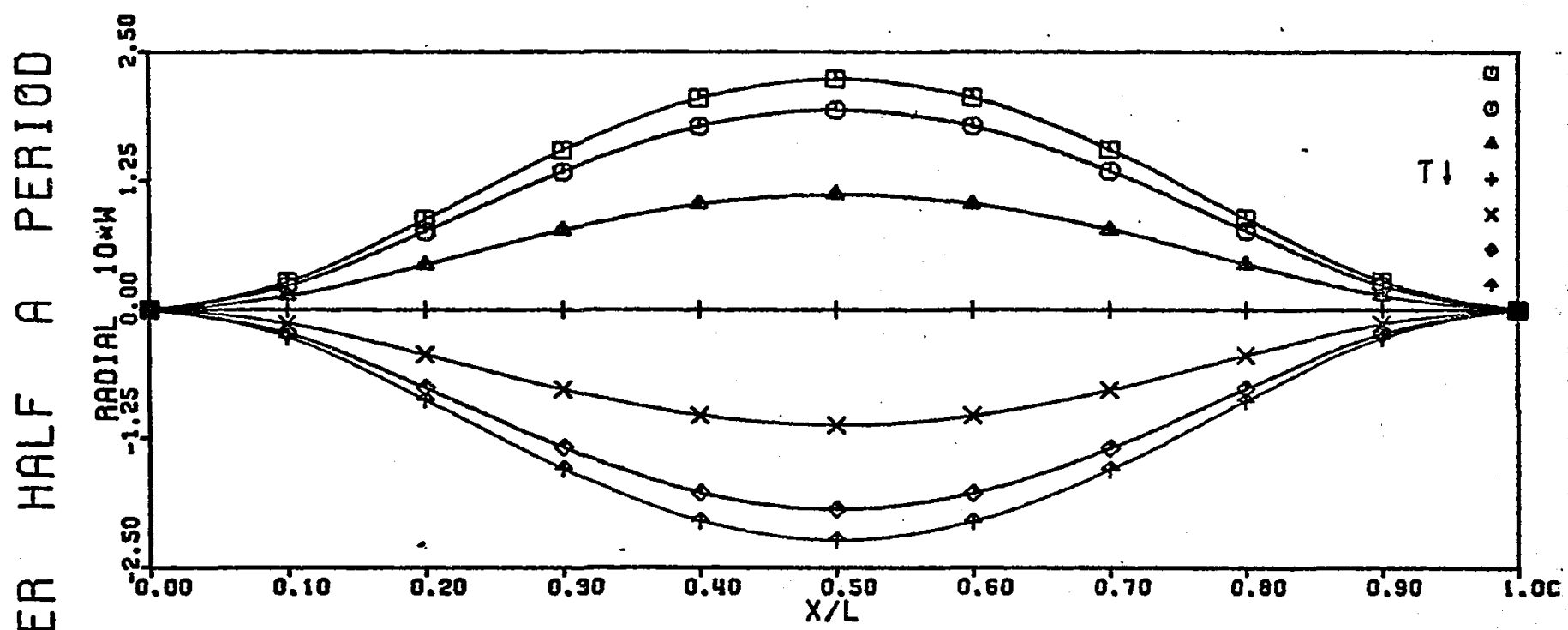

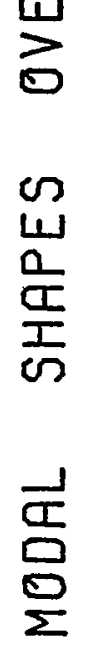

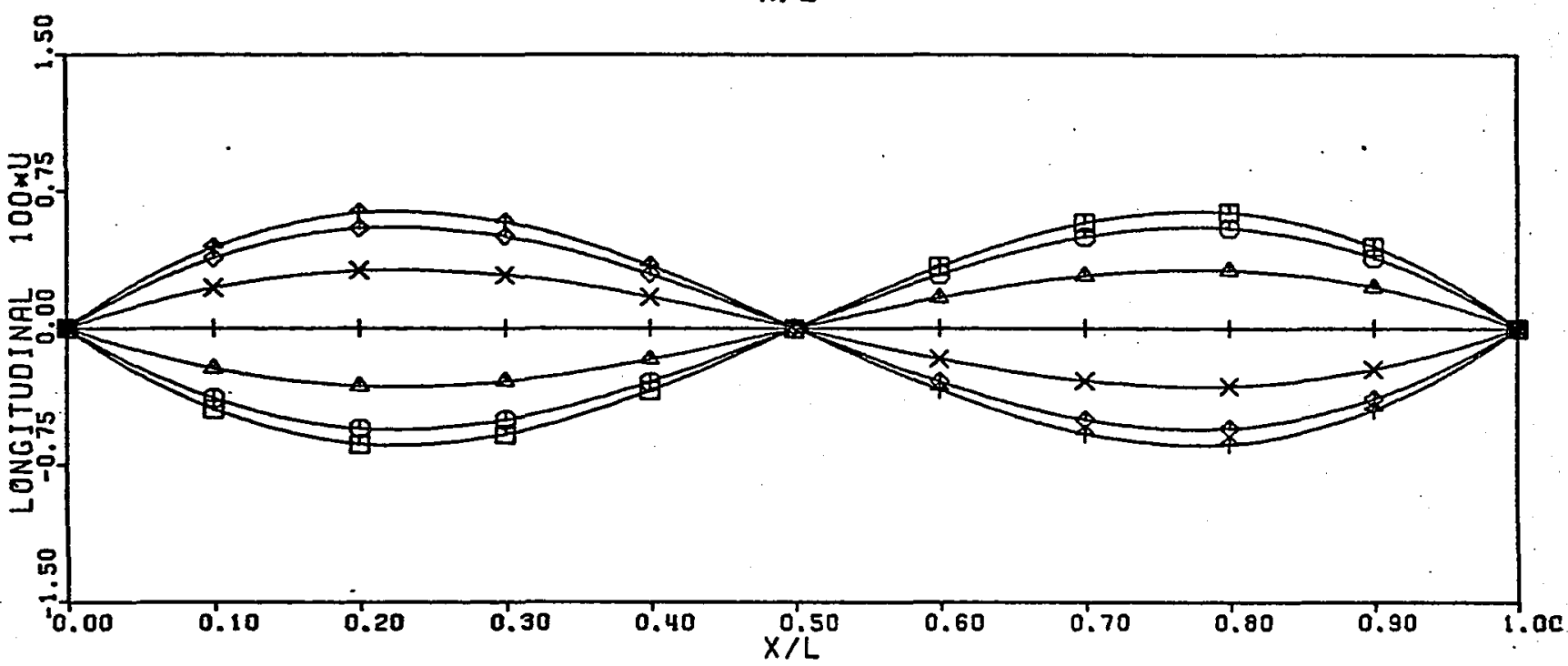

๒

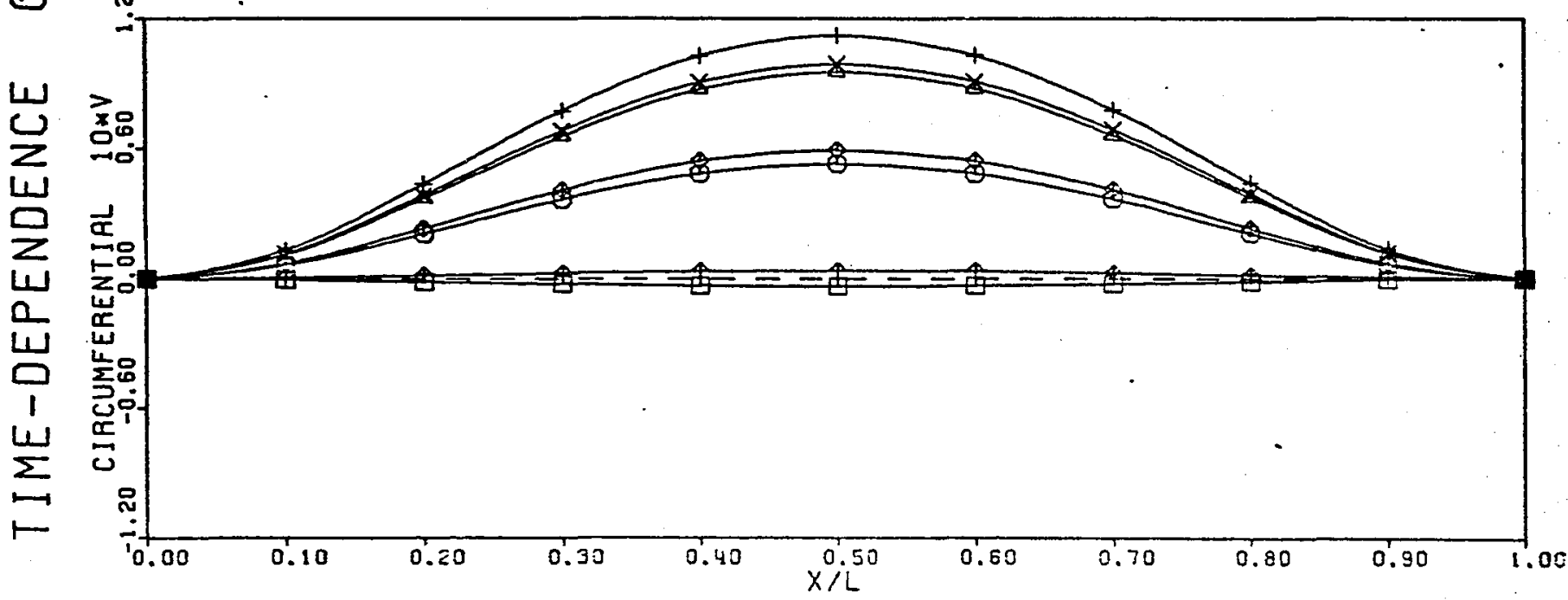


CIRCUMFERENTIAL WAVE NUMBER $\mathrm{N}=2$

NUMBER OF AXIAL HALF WAVES $M=1$

DIMENSIONLESS VELOCITY $\bar{U}=0.2$
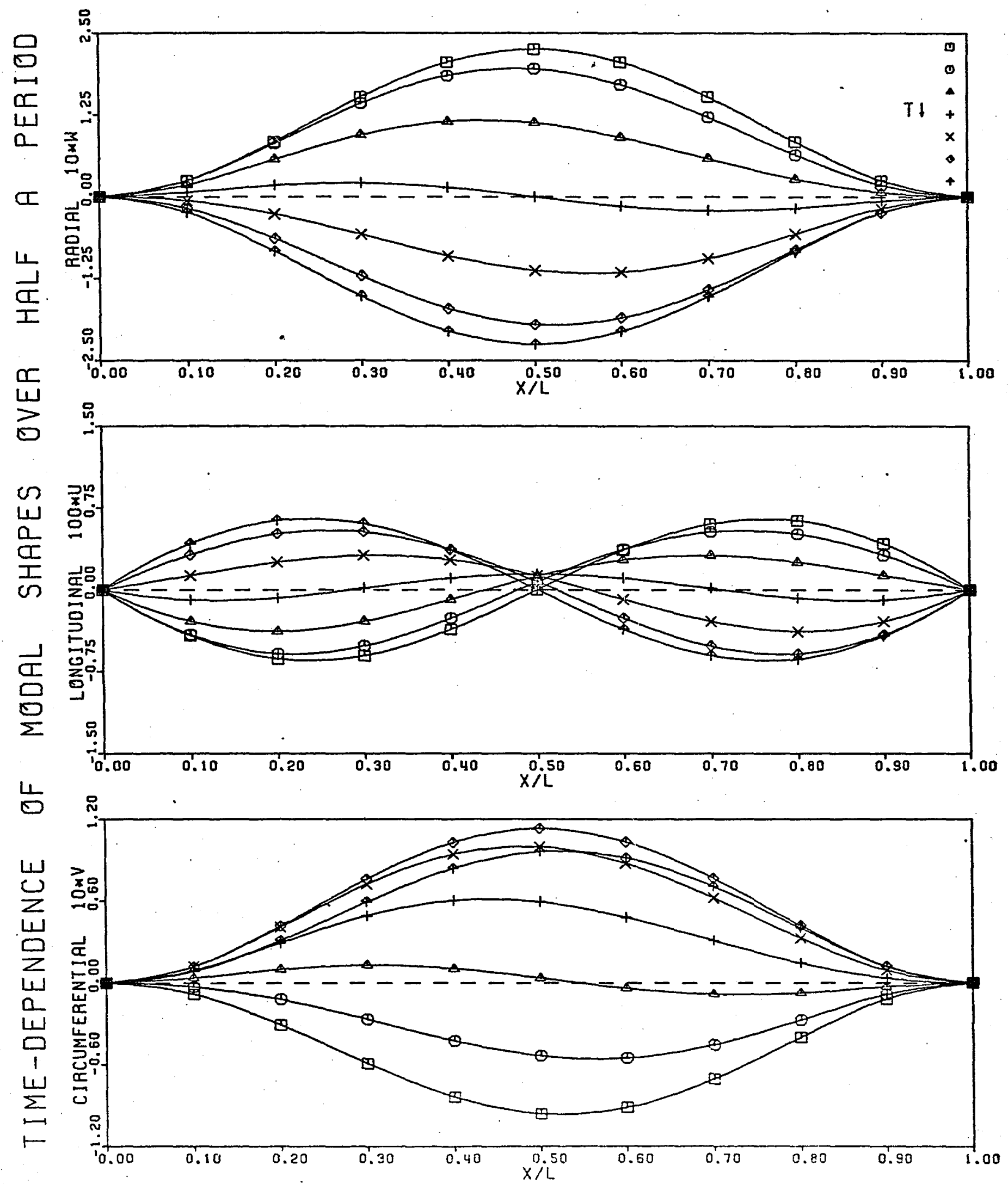
CIRCUMFERENTIAL WAVE NUMBER $\mathrm{N}=2$

NUMBER OF AXIAL HALF WAVES $M=1$

DIMENSIONLESS VELOCITY $\bar{U}=0.4$
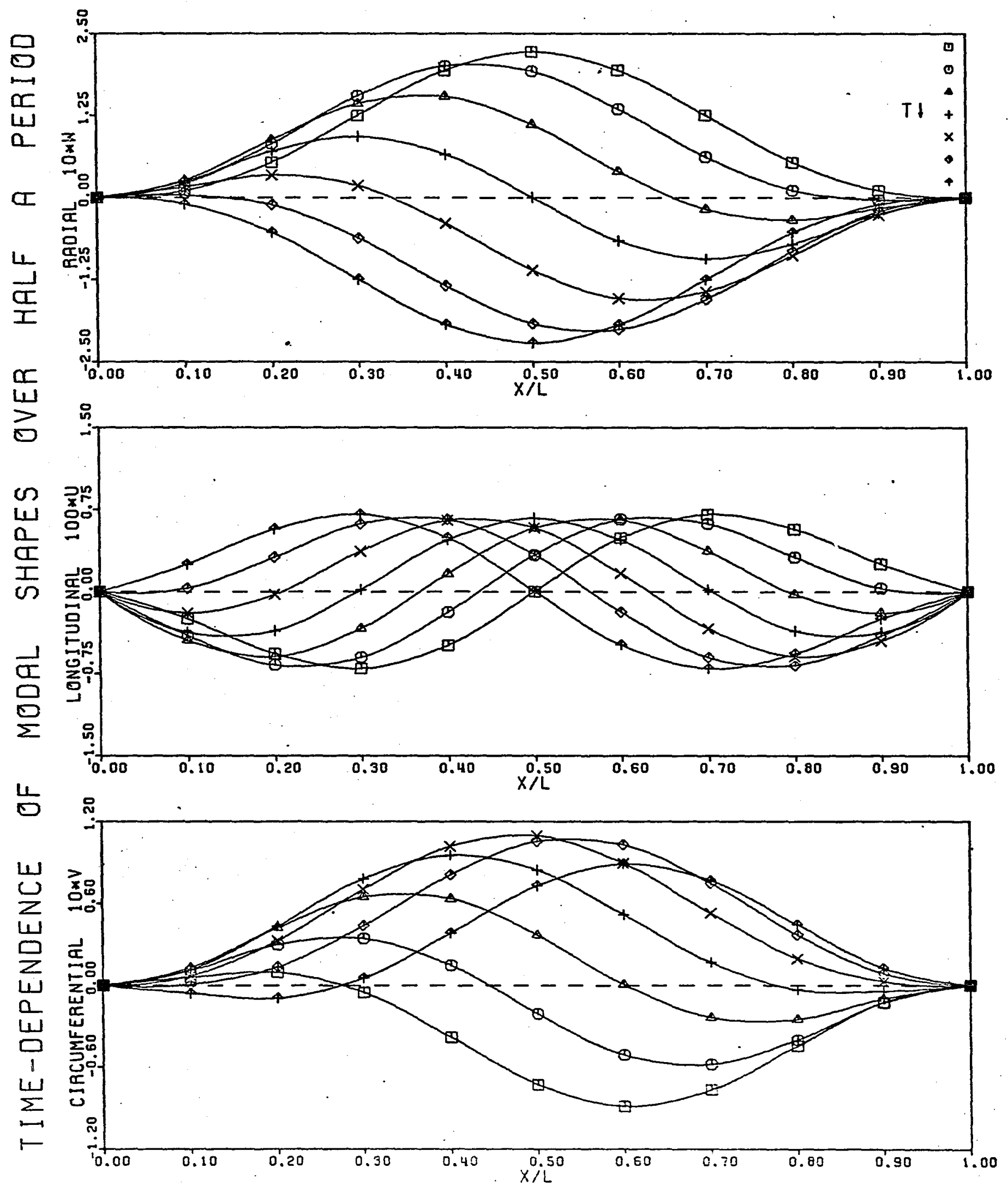
CIRCUMFERENTIAL WAVE NUMBER $N=2$

NUMBER OF AXIAL HALF WAVES $M=1$

DIMENSIONLESS VELOCITY $\bar{U}=0.5$

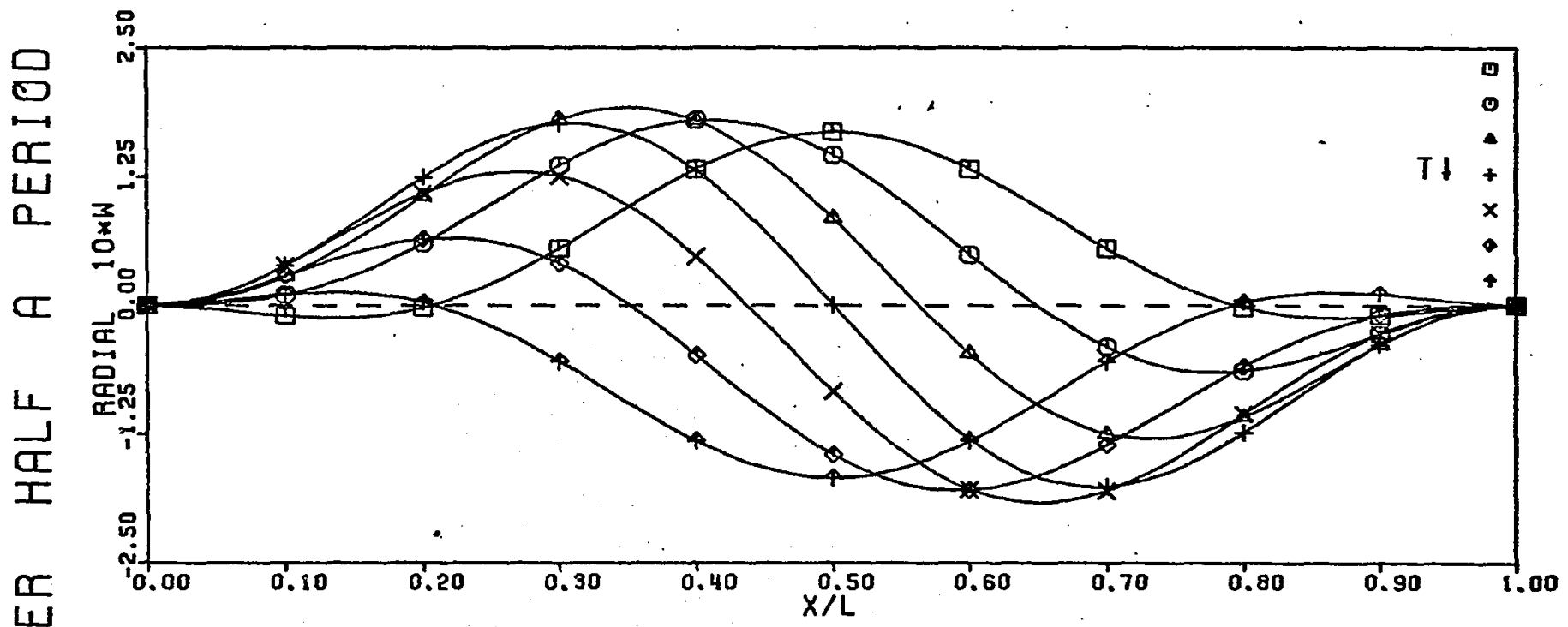

岂

w

in

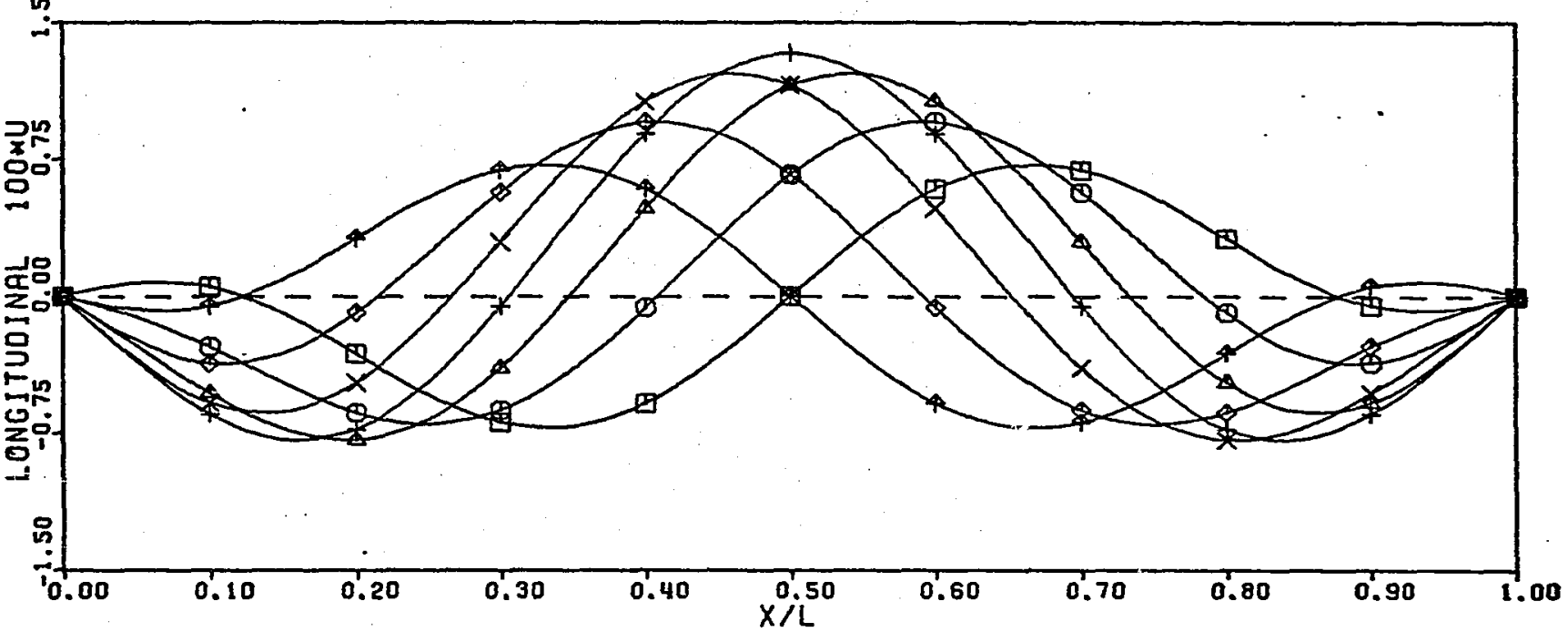

ธ

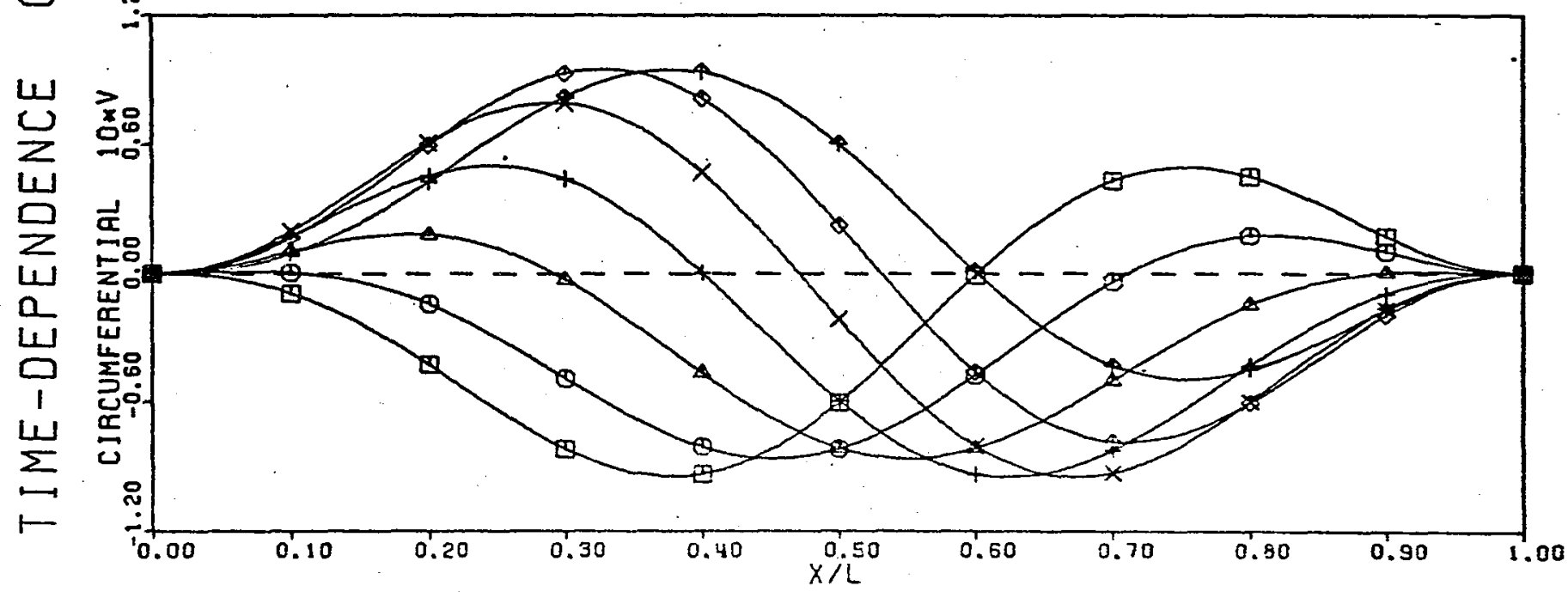


CIRCUMFERENT I AL WAVE NUMBER $N=2$ NUMBER OF AXIAL HALF WAVES $M=1$ DIMENSIONLESS VELOCITY $\bar{U}=\bar{U}_{B(-)}$

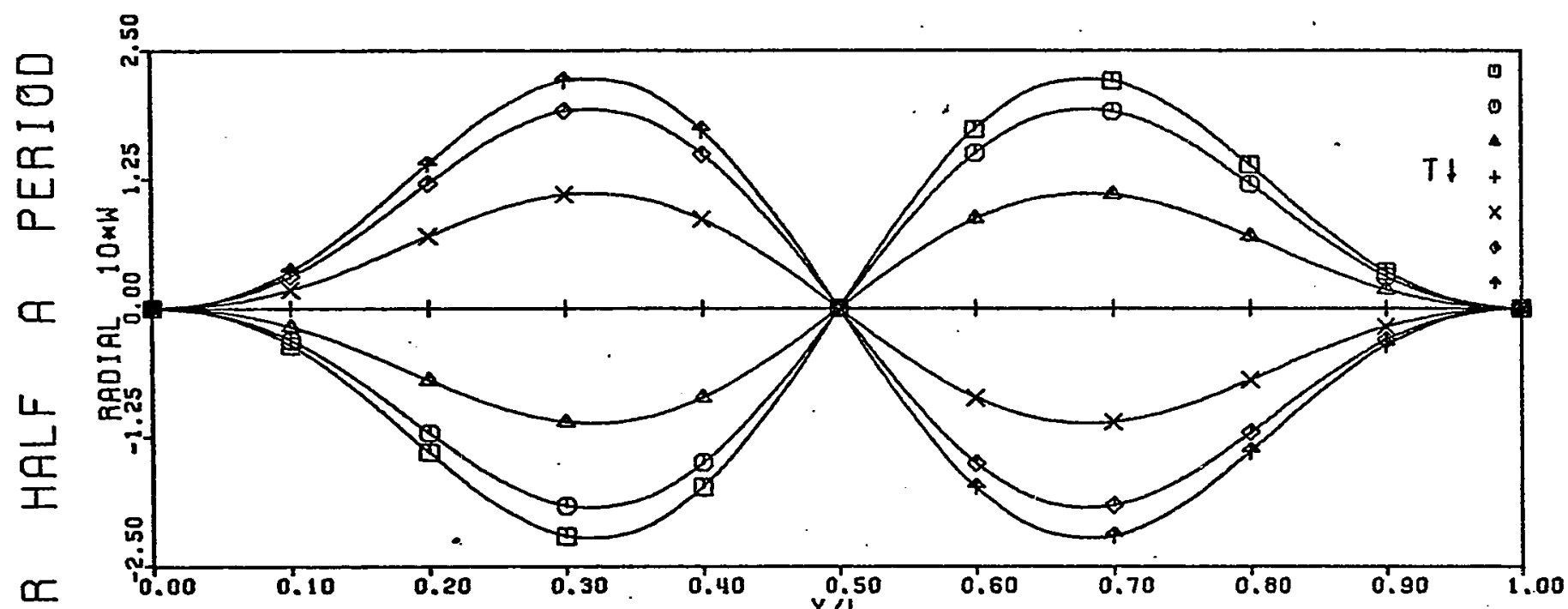

$\infty$
$\infty$

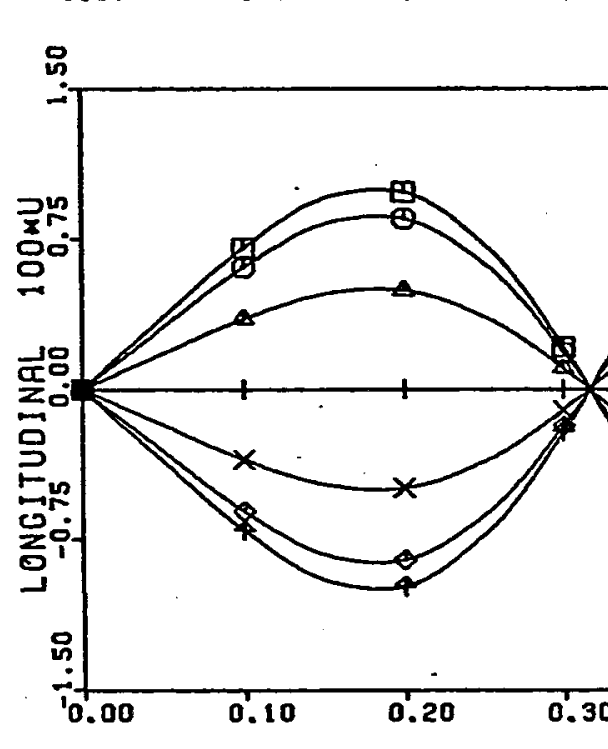

ए

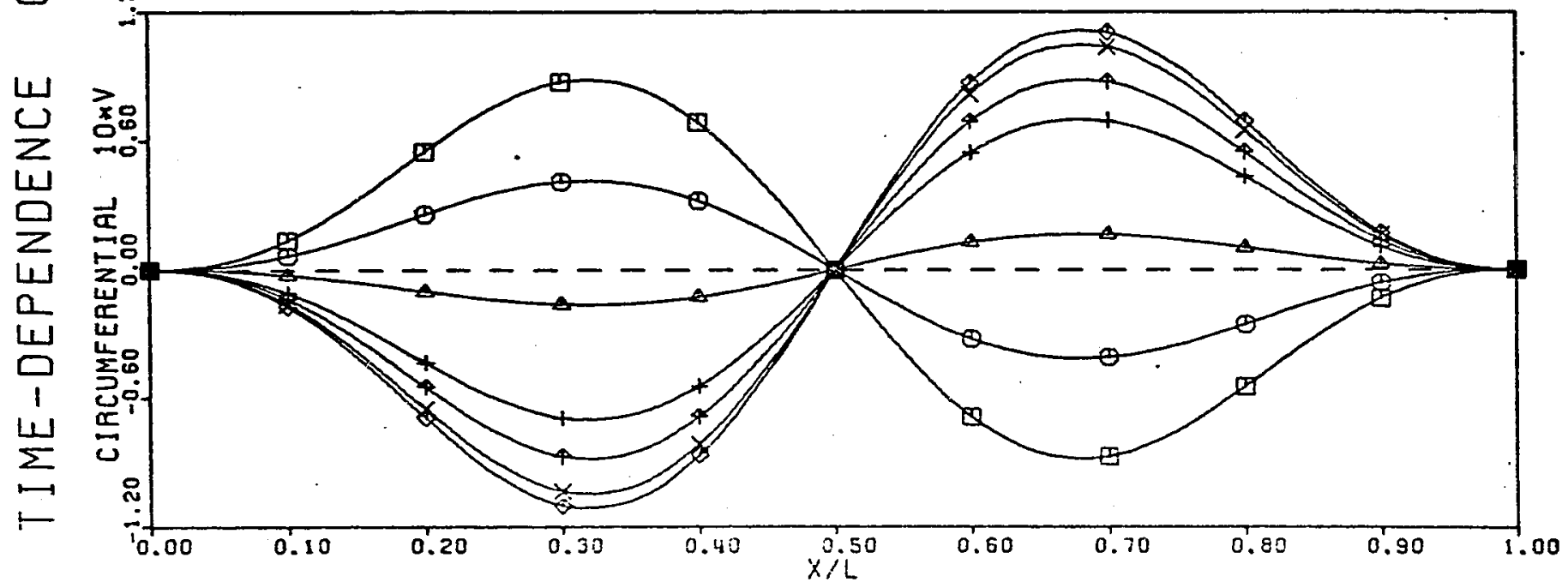


FIGURES $18 a, b, c, d, e-T i m e-d e p e n d e n c e$ of modal shapes over half a period, as function of dimensionless velocity, $\bar{U}$. Second axial mode at zero velocity of a clamped-clamped rubber tube.

$$
\begin{aligned}
& \mathrm{h} / \mathrm{a}=2.27 \times 10^{-2} \\
& \ell / a=25.9 \\
& \nu \quad=0.50 \\
& \mathrm{n}=2
\end{aligned}
$$


CIRCUMFERENTIAL WAVE NUMBER $N=2$

NUMBER OF AXIAL HALF WAVES $M=2$

DIMENSIONLESS VELOCITY $\overline{\mathrm{U}}=0.0$
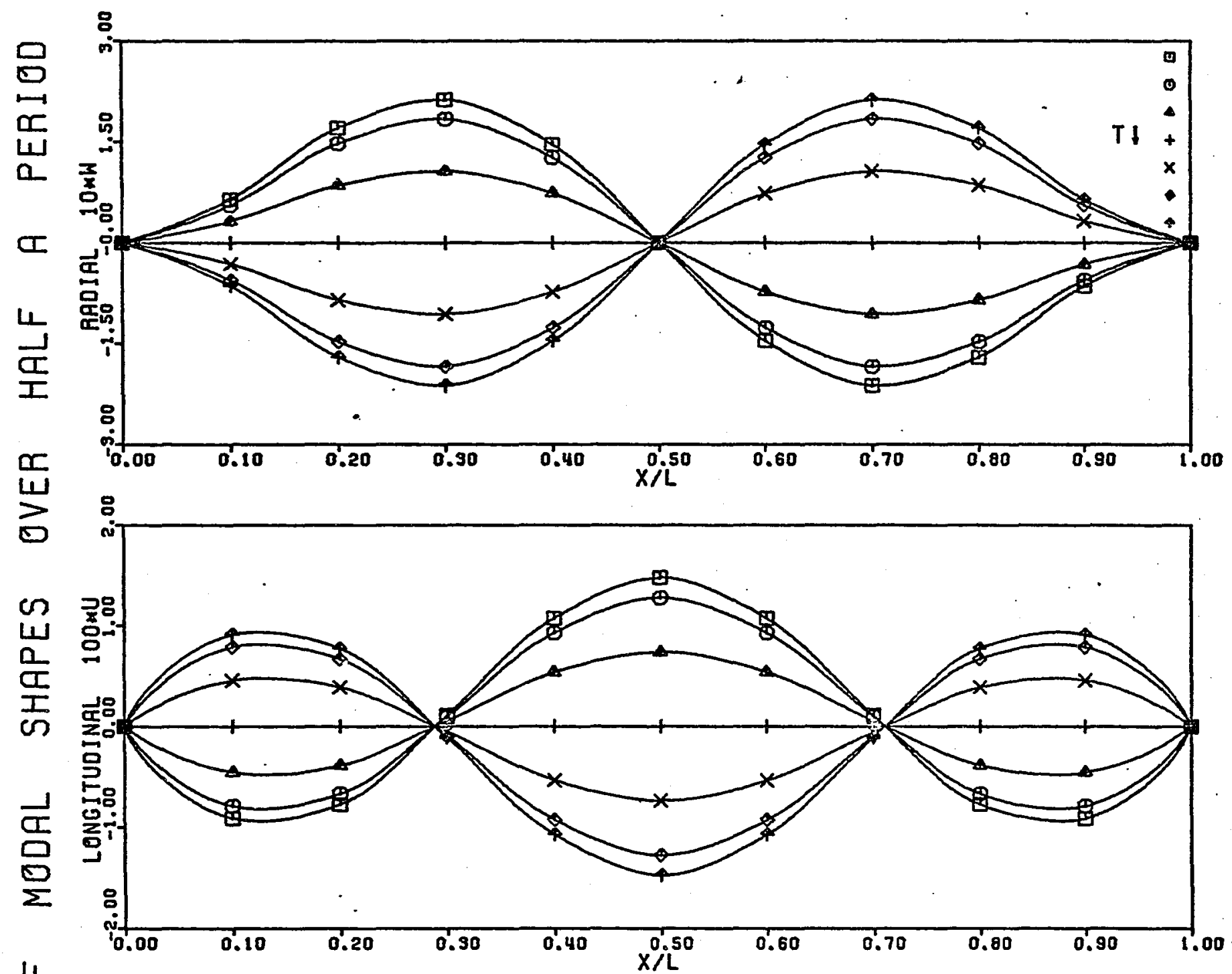

แ

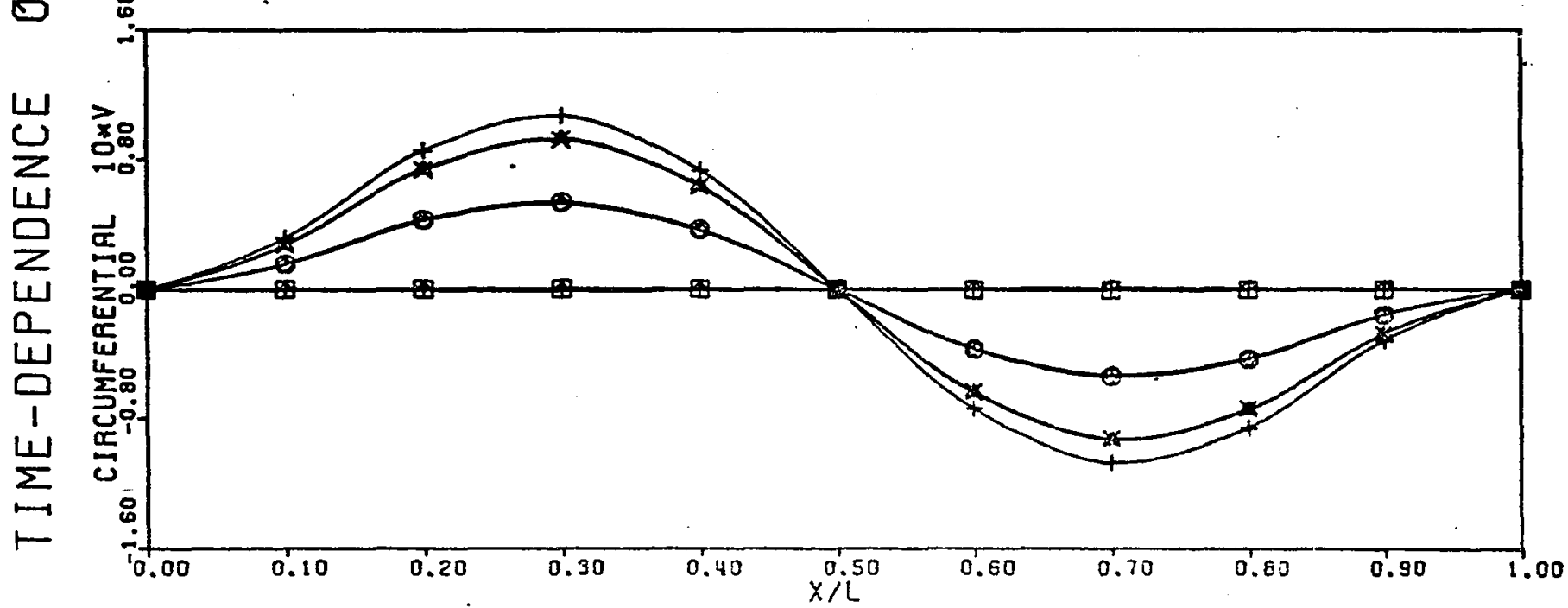


CIRCUMFERENTIAL WAVE NUMBER $\mathrm{N}=2$

NUMBER OF AXIAL HALF WAVES $M=2$

DIMENSIONLESS VELOCITY $\bar{U}=0.3$
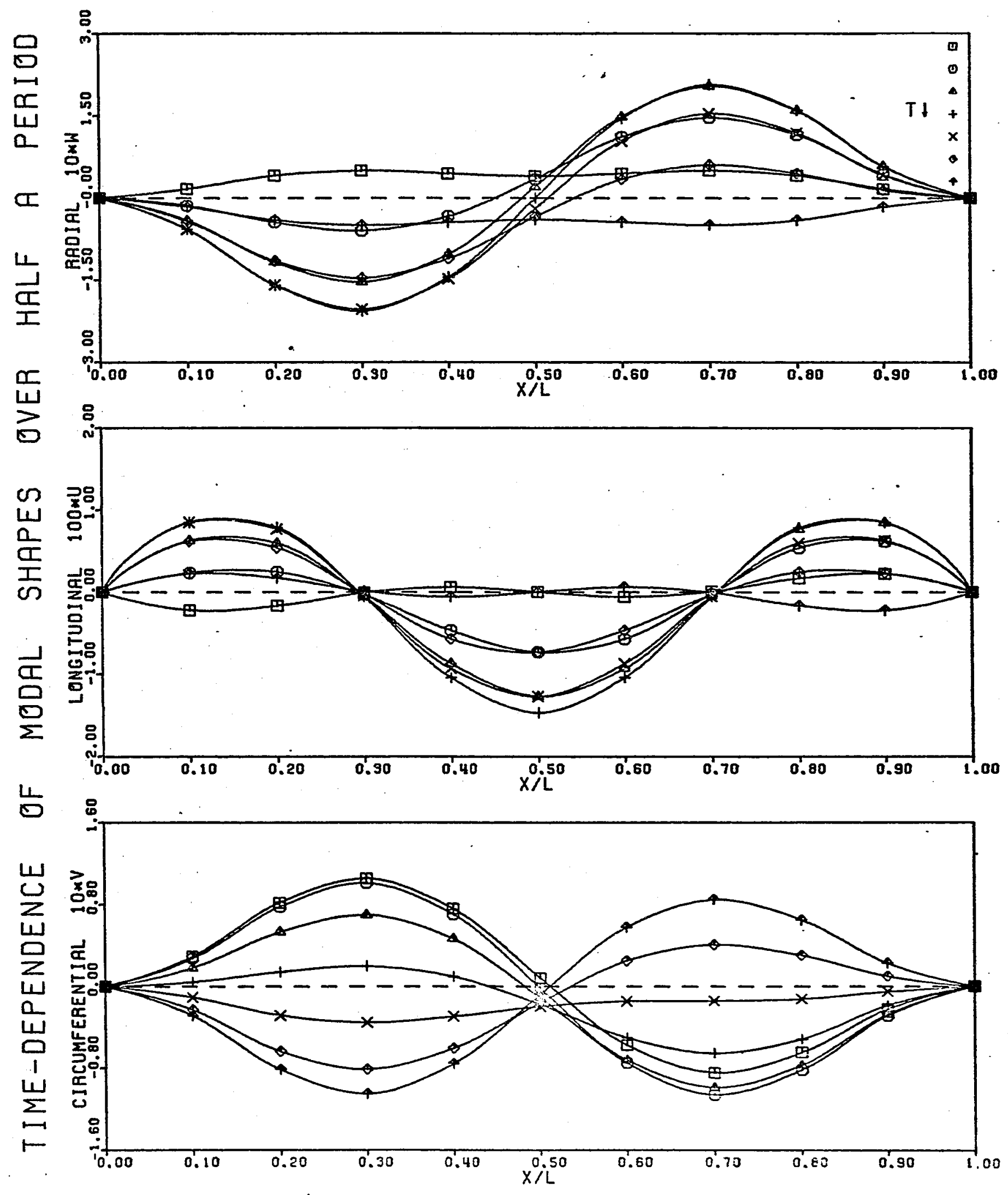
CIRCUMFERENTIAL WAVE NUMBER $N=2$

NUMBER OF AXIAL HALF WAVES $M=2$

DIMENSIONLESS VELOCITY $\bar{\nabla}=0.5$
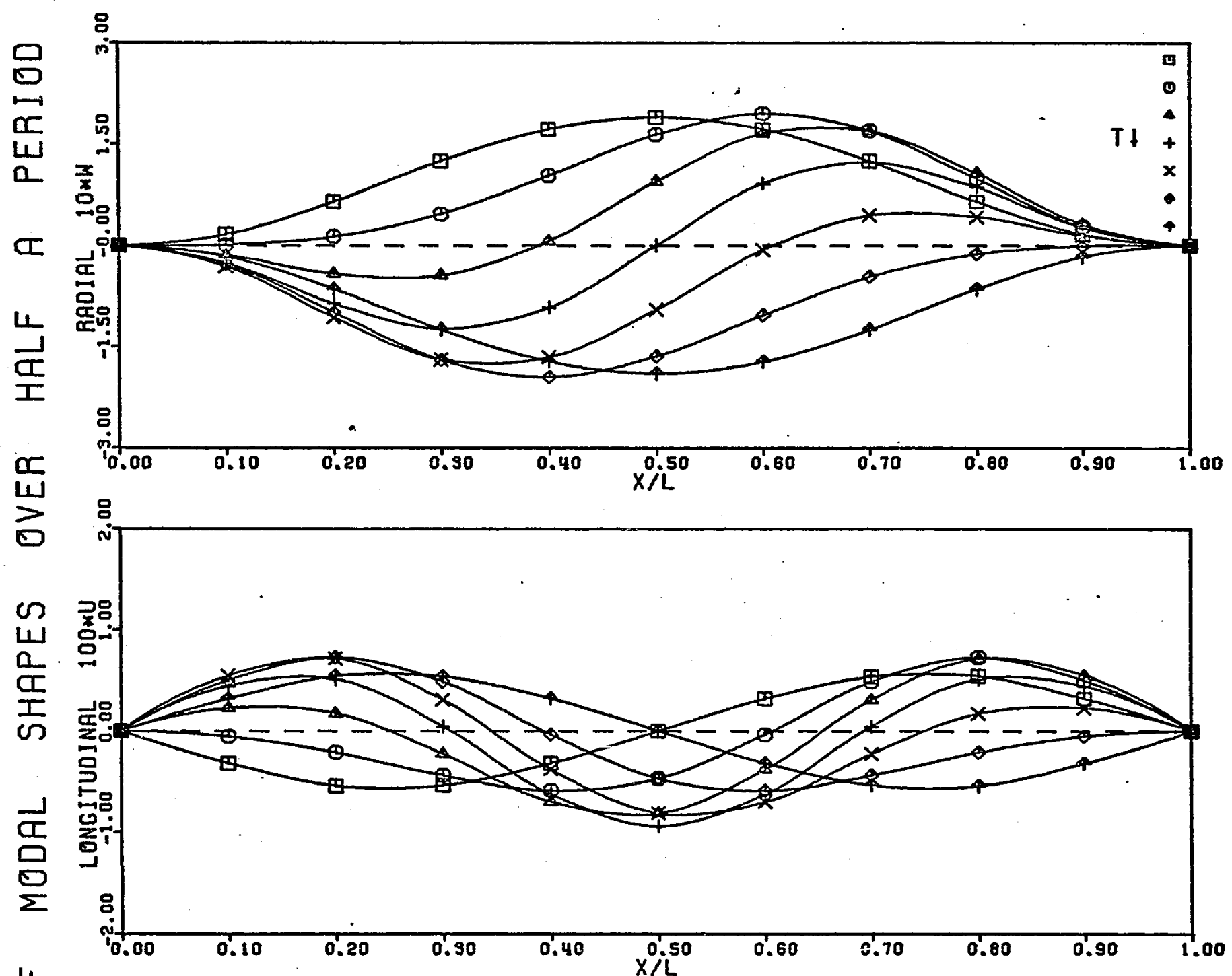

岁

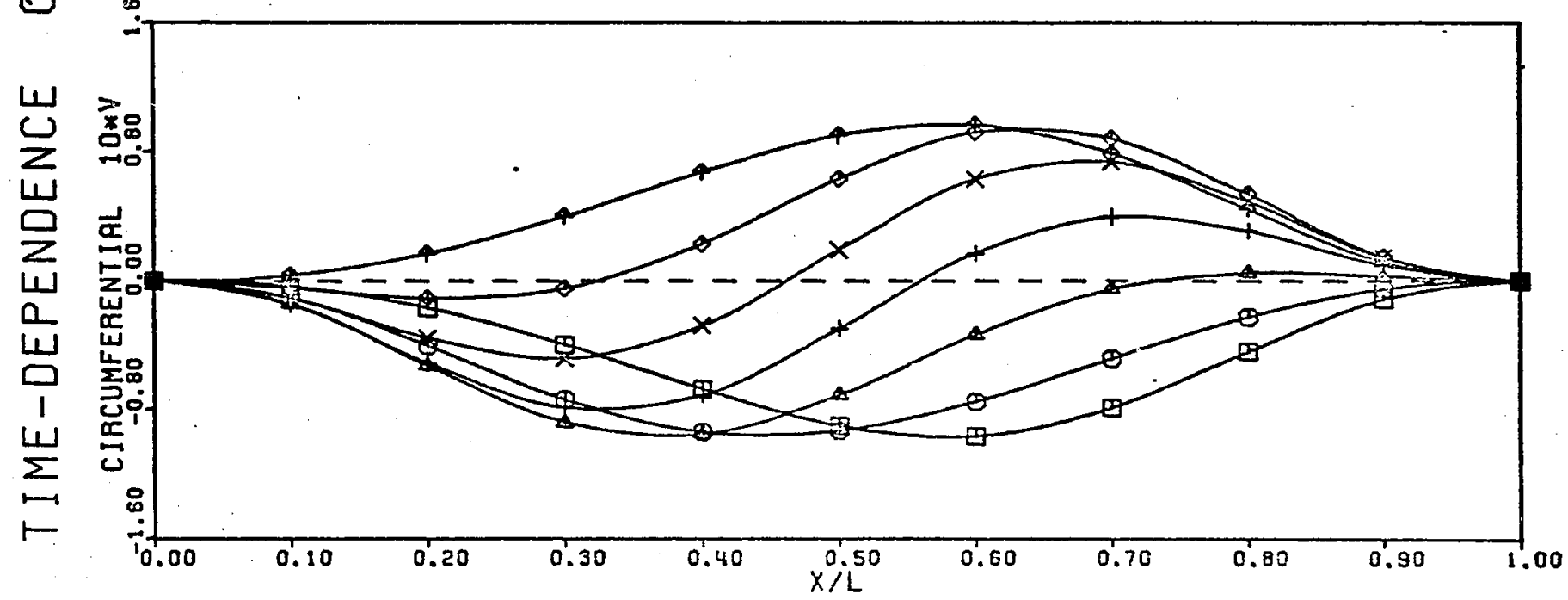


CIRCUMFERENTIAL WAVE NUMBER $N=2$

NUMBER OF AXIAL HALF WAVES $M=2$

DIMENSIONLESS VELOCITY $\bar{U}=0.6$
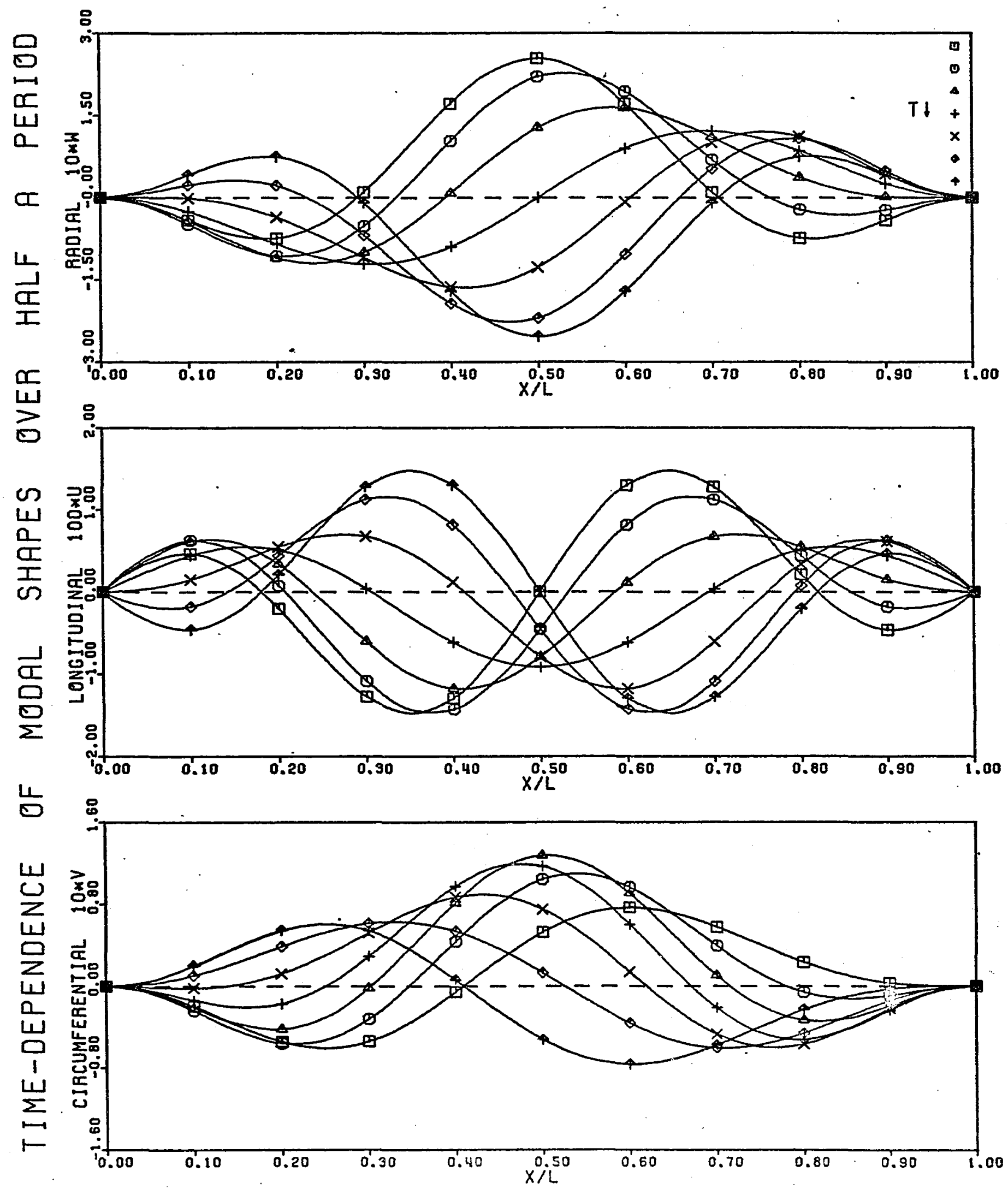
CIRCUMFERENTIAL WAVE NUMBER $N=2$

NUMBER OF AXIAL HALF WAVES $M=2$

DIMENSIONLESS VELOCITY $\bar{U}=\bar{U}_{\mathrm{B}(-)}$
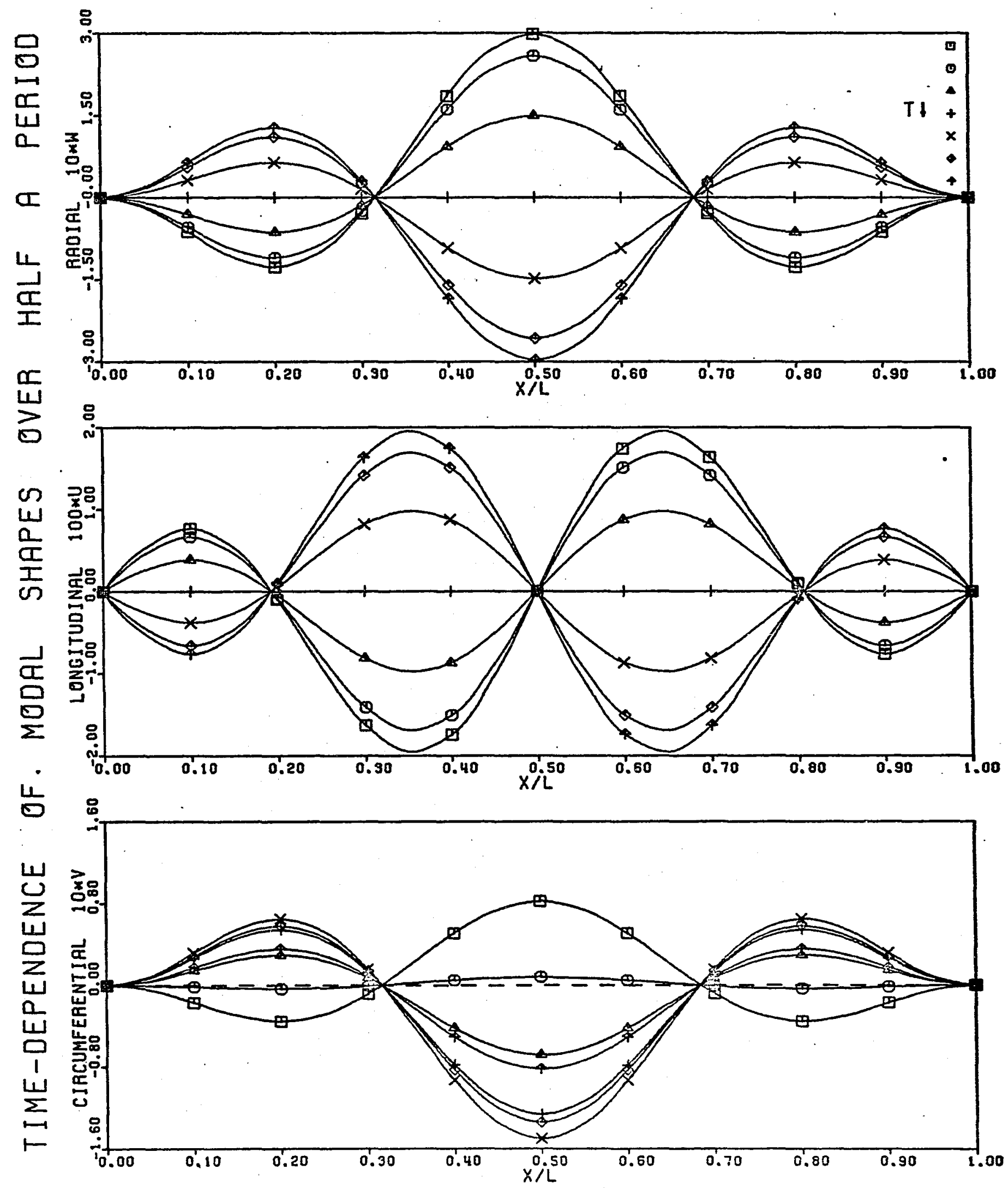
FIGURE 19 - Typical Argand diagram of dimensionless frequency, $\Omega$, as function of dimensionless velocity, $\bar{U}$, for a clamped-free rubber tube. First two axial modes for $\mathrm{n}=1,2,3$ and third axial mode for $\mathrm{n}=2$.

$$
\begin{aligned}
\mathrm{h} / \mathrm{a} & =2.27 \times 10^{-2} \\
\ell / a & =12.9 \\
\nu & =0.50 \\
\mathrm{n} & =1,2 \text { and } 3 \\
\mathrm{~m} & =1,2 \text { and } 3
\end{aligned}
$$




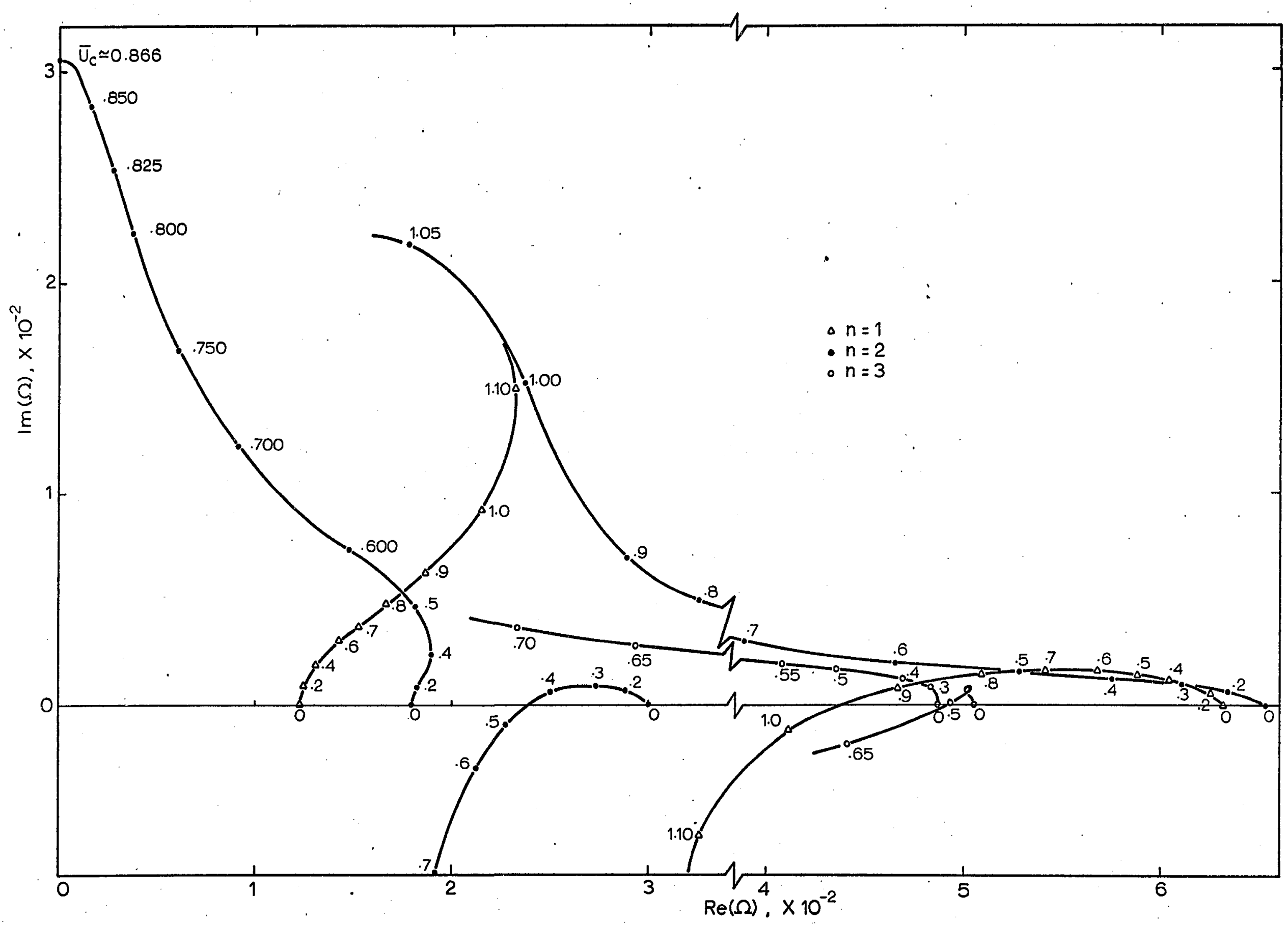




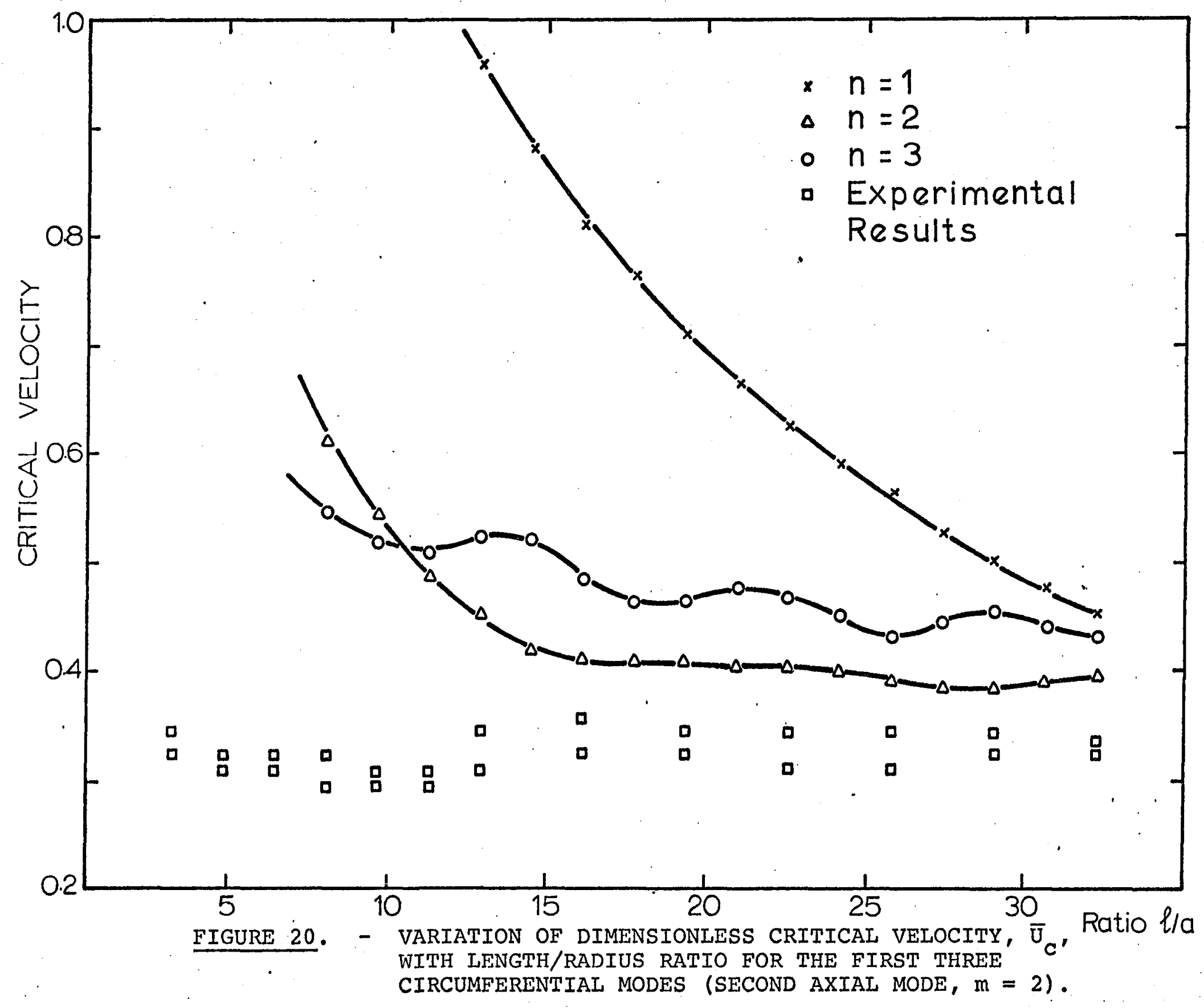




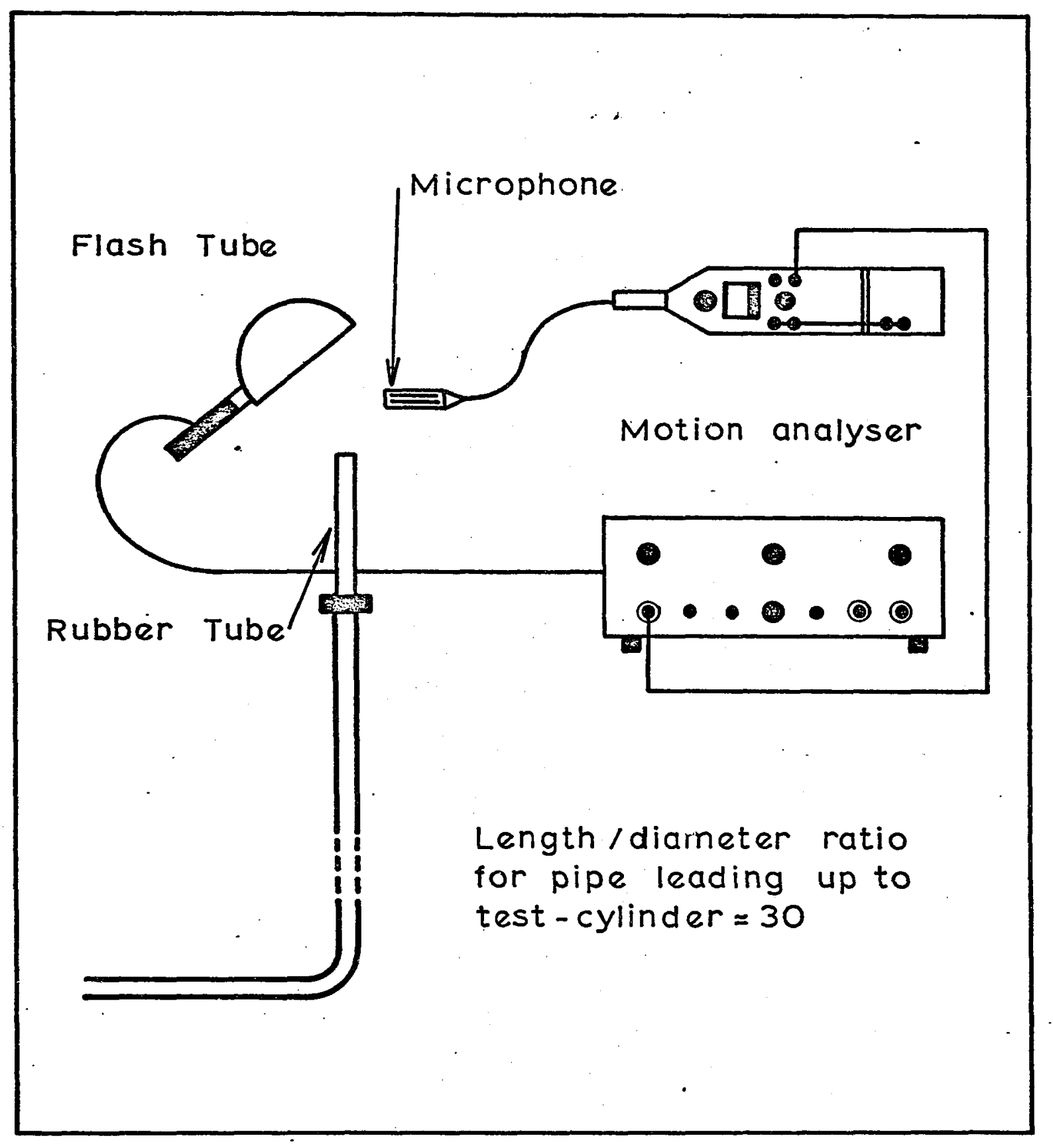

FIGURE 21. - SCHEMATIC DIAGRAM OF EXPERIMENTAL APPARATUS . 